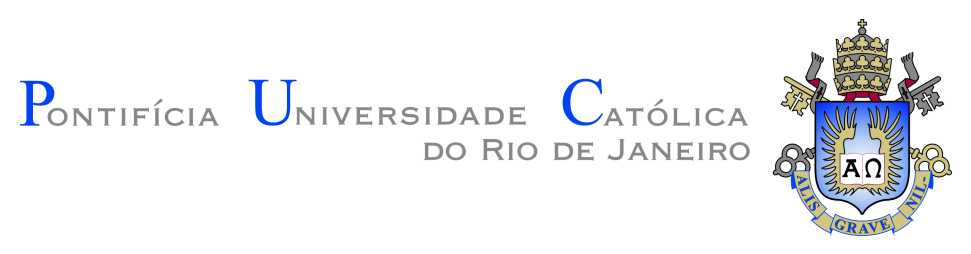

Francesco Pessina

\title{
Probing CP Violation and Physics Beyond the Standard Model in Neutrino Oscillation by New Generation Detectors
}

Tese de Doutorado

Thesis presented to the Programa de Pós-graduação em Física of PUC-Rio in partial fulfillment of the requirements for the degree of Doutor em Ciências - Física.

Advisor: Prof. Hiroshi Nunokawa 
All rights reserved.

\section{Francesco Pessina}

Received the title of Bachelor of Science degree in Physics from "Federico II' University (Naples, Italy, 2009), Master degree in Physics from "Federico II' University (Naples, Italy, 2012). He has specialized in the Theoretical Physics, High energy Physics and Quantum Gravity.

Bibliographic data

Pessina, Francesco

Probing CP Violation and Physics Beyond the Standard Model in Neutrino Oscillation by New Generation Detectors / Francesco Pessina; advisor: Hiroshi Nunokawa. - Rio de janeiro: PUC-Rio, Departamento de Física, 2019.

v., 119 f: il. color. ; $30 \mathrm{~cm}$

Tese (doutorado) - Pontifícia Universidade Católica do Rio de Janeiro, Departamento de Física.

Inclui bibliografia

1. Physics - Teses. 2. Oscillação de Neutrinos;. 3. Violação de $\mathrm{CP}$;. 4. Decaimento de Neutrinos;. 5. Neutrinos de Acelerador;. 6. Cintilador Liquido;. 7. Detetor Cherenkov de Água.. I. Nunokawa, Hiroshi. II. Pontifícia Universidade Católica do Rio de Janeiro. Departamento de Física. III. Título. 


\section{Acknowledgments}

This study was financed in part by the Coordenação de Aperfeiçoamento de Pessoal de Nível Superior - Brasil (CAPES) - Finance Code 001 and CNPq agency. I want also to thank Prof. Hiroshi and PUC-Rio University for the financial support provided to me in the last semester. 


\section{Abstract}

Pessina, Francesco; Nunokawa, Hiroshi (Advisor). Probing CP Violation and Physics Beyond the Standard Model in Neutrino Oscillation by New Generation Detectors. Rio de Janeiro, 2019. 119p. Tese de doutorado - Departamento de Física, Pontifícia Universidade Católica do Rio de Janeiro.

In this thesis we will study a novel method to measure the leptonic $\mathrm{CP}$ violation phase, $\delta_{C P}$, in an experimental set up called LiquidO, and the possibility by the future experiment Hyper-Kamiokande to put a limit on the neutrino lifetime. Both experiment are accelerator based ones that will use the well established neutrino flavour oscillation phenomenon to perform their measurement. The first experimental set up uses two new features: firstly it uses as a source a flux of $\nu_{\mu}$ and $\bar{\nu}_{\mu}$ coming from pions $\left(\pi^{+}\right)$ decay at rest with a baseline of $16 \mathrm{~km}$ and secondly it uses a new detection method. This new detection is performed using opaque Liquid Scintillator (LS) with optical fibers that allows $e^{+} e^{-}$identification. We will discuss the phenomenological main characteristics of this set up and we will calculate the expected significance to exclude the $\delta_{C P}=(0, \pi)$ hypothesis, the $1 \sigma$ precision of the $\delta_{C P}$ measurement and also the expected allowed regions in the $\sin ^{2} \theta_{23}-\delta_{C P}$ plane. For what it concerns the second part of our work, we will focus on the Hyper-Kamiokande experiment, upgrade of the 2015 Nobel prize awarded Super-Kamiokande. This is a water Cherenkov detector that will use a $\nu_{\mu}\left(\bar{\nu}_{\mu}\right)$ flux with a typical neutrino energy $<10 \mathrm{GeV}$ coming from the JPARC facility with a baseline of $295 \mathrm{~km}$. We will call this source-detector configuration T2HK to distinguish T2HKK, the possible extension of this experiment in Korea that will use the same beam but it will be located at $1100 \mathrm{~km}$ from the source. We will briefly introduce the neutrino decay mode that can be classified in two types: one is what is called "invisible decay" , i.e. when neutrino decays into a sterile neutrino state plus a scalar particle, and the other is called "visible decay", i.e. when neutrino decays into an active mass eigenstate plus a scalar particle. We will calculate the limit on the $\nu_{3}$ lifetime for the invisible and the visible case for both configurations T2HK and T2HKK.

\section{Keywords}

Neutrino Oscillation; CP Violation; Neutrino Decay; Accelerator Neutrino; Liquid Scintillator; Water Cherenkov Detector. 


\section{Resumo}

Pessina, Francesco; Nunokawa, Hiroshi (Orientador). Estudo de Violação de CP e Física Além do Modelo Padrão através de Oscilação de Neutrinos em Detetores de Nova Geração. Rio de Janeiro, 2019. 119p. Tese de Doutorado - Departamento de

Física, Pontifícia Universidade Católica do Rio de Janeiro.

Neste trabalho de tese investigaremos um novo método para medir a fase leptóonica de violação $\mathrm{CP}, \delta_{C P}$, com um set up experimental chamado LiquidO e, estudaremos a possibilidade do futuro experimento HyperKamiokande de pôr um limite sobre o tempo de vida do neutrino. Ambos são experimentos que detectam neutrinos de acelerador que performarão as próprias medições através do estudo do bem conhecido fenómeno da oscilação de sabor de neutrinos. O primeiro experimento considerado possui duas novas características: primeiramente utiliza como fonte de neutrino um fluxo de $\nu_{\mu}$ e $\bar{\nu}_{\mu}$ produzidos por pions $\left(\pi^{+}\right)$em repouso a uma distância de $16 \mathrm{~km}$ e secondariamente vai usar uma nova forma de detecção. Essa detecção é feita utilizando cintilador liquido opaco com fibras ópticas que permitem distinguir entre $e^{+}$e $e^{-}$. Nós explicaremos as principais propriedades fenomenológicas desta configuração e calcularemos a significância estatística de exclusão da hipótese $\delta_{C P}=(0, \pi)$, a precisão de medição de $\delta_{C P}$ com $1 \sigma$ de confiança estatística e também as regões permitidas no espaço dos parámetros $\sin ^{2} \theta_{23}-\delta_{C P}$. Na segunda parte do nosso trabalho, nos concentraremos no experimento Hyper-Kamiokande, versão melhorada do experimento Super-Kamiokande que se adjudicou o prémio Nobel em 2015. Este é um detector de luz Cherenkov que utiliza um fluxo de $\nu_{\mu}\left(\bar{\nu}_{\mu}\right)$ com energias $<10 \mathrm{GeV}$ produzido no acelerador JPARC e colocado a uma distância de $295 \mathrm{~km}$. Neste trabalho identificaremos este experimento como T2HK enquanto para a sua estensão na Korea, que utilizará a mesma fonte mas será colocada a $1100 \mathrm{~km}$ de distância, utilizaremos a sigla T2HKK. Nós introduziremos brevemente as modalidades de decaimento do neutrino que podem ser classificadas em dois tipos: um é chamado decaimento invisível, ou seja, quando o neutrino de origem decai em um estado estéril mais uma partícula escalar, e o outro e chamdo de decaimento visível, ou seja, quando o neutrino de origem decai em um autoestado de massa ativo mais uma partícula escalar. Em fim calcularemos as sensitividades no limite da vida média do autoestado $\nu_{3}$ para os casos de decaimento visível e invisível para as configurações de T2HK e T2HKK.

\section{Palavras-chave}


Oscillação de Neutrinos; Violação de CP; Decaimento de Neutrinos; Neutrinos de Acelerador; Cintilador Liquido; Detetor Cherenkov de Água. 


\section{Table of contents}

1 Introduction $\quad 16$

2 Neutrinos: Who are They? $\quad 17$

2.1 The Standard Model $\quad 17$

2.2 The Electroweak Interaction 19

2.3 Neutrino Interactions: The Inverse Beta Decay 26

2.4 Neutrino Oscillation in Vacuum 30

2.5 Neutrino Oscillation in Matter 35

2.5.1 Two Generation Case: The MSW Effect 37

2.6 Leptonic CP Violation in Neutrino Oscillation Framework 40

3 Current Status of Neutrino Physics 43

3.1 Solar Neutrinos 43

3.2 Atmospheric and Accelerator Neutrinos 47

3.3 Reactor Neutrinos 54

3.4 Oscillation Parameter: Global Analysis 58

4 A Novel Method to Measure the Leptonic CP-asymmetry: Experimental Set Up $\quad 61$

4.1 Neutrino Coming From $\pi^{+}$Decay At Rest Chain 63

4.2 Neutrino Oscillation Probability $P\left(\nu_{\mu} \rightarrow \nu_{e}\right) \quad 68$

4.3 Events Number for Neutrinos and Anti-Neutrinos 70

4.4 The Physical Background $\quad 74$

5 Sensitivity Study: $\chi^{2}$ Definition $\quad 76$

5.1 Background Effects on Sensitivity $\quad 79$

5.2 B and $\eta$ Scaling 81

5.3 Final Results 82

6 Probing Neutrino Decay Scenarios with HK Detector 85

6.1 Phenomenology of Neutrino Decay 85

6.1.1 Invisible Decay $\quad 86$

6.1.1.1 Disappearance modes $\nu_{\mu} \rightarrow \nu_{\mu}$ and $\bar{\nu}_{\mu} \rightarrow \bar{\nu}_{\mu} \quad 87$

6.1.1.2 Appearance modes $\nu_{\mu} \rightarrow \nu_{e}$ and $\bar{\nu}_{\mu} \rightarrow \bar{\nu}_{e} \quad 88$

6.1.2 Visible Decay $\quad 89$

6.1.2.1 Disappearance modes $\nu_{\mu} \rightarrow \nu_{\mu}$ and $\bar{\nu}_{\mu} \rightarrow \bar{\nu}_{\mu} \quad 91$

6.1.2.2 Appearance modes $\nu_{\mu} \rightarrow \nu_{e}$ and $\bar{\nu}_{\mu} \rightarrow \bar{\nu}_{e} \quad 91$

6.2 Experimental setup and assumptions for our analysis 93

6.2.1 Hyper-Kamiokande 94

6.2.2 Second Hyper-Kamiokande Detector in Korea 96

6.3 Definition of $\chi^{2}$ Function 96

$\begin{array}{ll}6.4 \text { Analysis Results } & 99\end{array}$

6.4.1 Visible Case $\quad 99$

7 Conclusions 103 
$\begin{array}{ll}\text { A Higgs Mechanism and Flavour Mixing } & 104\end{array}$

B Visible Decay Calculation $\quad 109$

C Calculations of Number of Events for T2HK and T2HKK 113

$\begin{array}{ll}\text { Bibliography } & 116\end{array}$ 


\section{List of figures}

Figure 2.1 Feynman diagrams representation of the charged current interaction vertex of the first lepton generation.

Figure 2.2 Feynman diagrams representation of the neutral current and electromagnetic interaction vertex of the first lepton generation.

Figure 2.3 Contraction of the $W$ and $Z$ gauge boson propagator in a generic low-energy CC process. Figure from [2]

Figure 2.4 Tree-level Feynman diagram of the inverse neutron decay process in (2-40), with the neutron and proton represented as a bound states of valence quarks. The blobs represent strong interaction binding. Figure edited from [2].

Figure 2.5 A plot of the function (2-45) in the range of $E_{\nu}=$ $[0,52.8] \mathrm{MeV}$.

Figure 2.6 Neutrino flavour change (oscillation) in vacuum. "Amp" denotes the amplitude of the process.

Figure 2.7 Pictorial representation of neutrino squared-mass spectral pattern. The "??" represent the unknown value of the neutrino mass scale. Figure from [9].

Figure $2.8 P\left(\nu_{\mu} \rightarrow \nu_{\mu}\right), P\left(\nu_{\mu} \rightarrow \nu_{e}\right)$ and $P\left(\nu_{\mu} \rightarrow \nu_{\tau}\right)$ in vacuum at a fixed distance $L=1100 \mathrm{~km}$.

Figure 2.9 Feynman diagrams of the coherent forward elastic scattering processes that generate the $C C$ potential $V_{C C}$ through $\mathrm{W}$ exchange and the $N C$ potential $V_{N C}$ through $Z$ exchange. Figure taken from [2].

Figure 2.10 Scheme of the CPT, CP, and T transformations that relate different flavour transition channels.

Figure 3.1 The neutrino fluxes are given in units of $\mathrm{cm}^{-2} \mathrm{~s}^{-1} \mathrm{MeV}^{-1}$ for continuum spectra and $\mathrm{cm}^{-2} \mathrm{~s}^{-1} \mathrm{MeV}^{-1}$ for line spectra. This figure is taken from [8].

Figure 3.2 Dependence of the probability $P_{e e}$ integrated over the day and the night time periods. This figure is taken from [15].

Figure 3.3 The right panel shows the allowed regions of $\theta_{12}$ and $\Delta m_{21}^{2}$ from the global fit of the solar neutrino data (red) as well as KamLAND (blue), for $\theta_{13}$ fixed to the best fit of the reactor experiments. In the left panel are shown also regions restricted by individual experiments (Chlorine, Gallex, Borexino). This figure is taken from [15].

Figure 3.4 Neutrino flavour ratios calculated with the all-direction and one-year averaged atmospheric neutrino fluxes at Kamioka. This figure is taken from [8].

Figure 3.5 Zenith angle distributions for multi-GeV atmospheric neutrino events presented at the 18th International Conference on Neutrino Physics and Astrophysics (Neutrino'98) by the Super-Kamiokande collaboration. This figure is taken from [20]. 
Figure 3.6 Zenith angle distributions for the $\tau$-like events selected from the data observed in Super-Kamiokande. Circles with error bars show the data. Solid histograms show the Monte Carlo prediction with $\nu_{\mu} \rightarrow \nu_{\tau}$ oscillations but without the charged current $\nu_{\tau}$ interactions. The gray histograms show the fit result including the $\nu_{\tau}$ interactions.. This figure is taken from [20].

Figure 3.7 Results of the Super-K fit to three-neutrino oscillations. The plot shows the $\left|\Delta m_{32}^{2}\right|$ versus $\sin ^{2} \theta_{23}$ plane assuming an inverted mass hierarchy and a star indicates the best fit point. This figure is taken from [19].

Figure 3.8 Allowed regions from the ANTARES and IceCube neutrino oscillation results compared with those of MINOS and Super- Kamiokande. This figure is taken from [19].

Figure 3.9 Here we show the most recent results of the T2K collaboration. In this figure we have the allowed region for $\Delta m_{32}^{2}$ and $\theta_{23}$ for both IO and NO ordering at 1 and $2-\sigma$ confidence level (bottom-left), and the $2-\sigma$ allowed region for the CP $\delta$ values (bottom-right). We also show the cumulative statistics for the different run (top-left) and the best fit values for $\Delta m_{32}^{2}$ and $\theta_{23}$ in IO and NO hypothesis. This figure is taken from [21].

Figure 3.10 Here we show the most recent results of the NOvA collaboration. In this figure we have the allowed region for $\Delta m_{32}^{2}$ and $\theta_{23}$ for both IO and NO ordering at 1 and $2 \sigma$ confidence level (left), and the confidence level of the allowed region for the CP $\delta$ values (top-right). We also show the the best fit values for $\Delta m_{32}^{2}$ and $\theta_{23}$ for the best fit NO hypothesis (bottom-right). This figure is taken from [22].

Figure 3.11 Inverse beta decay yields from the convolution of the IBD cross section and the antineutrino spectra for ${ }^{235} \mathrm{U},{ }^{238} \mathrm{U}$, ${ }^{239} \mathrm{Pu}$, and ${ }^{241} \mathrm{Pu}$. This figure is taken from [25].

Figure 3.12 Pictorial representation of the IBD detection in a Gdloaded liquid scintillator. The $\bar{\nu}_{e}$ interacts with a free proton producing a $e^{+}$. The annihilation of the positron represent the prompt signal otherwise the recoil neutron capture on $\mathrm{Gd}$ (or $\mathrm{H})$ represent the delayed signal. This figure is taken from [25].

Figure 3.13 Global result of $\theta_{13}$ (left) and $\Delta m_{32}^{2}$ (right) taken from the results presented at the Neutrino 2018 conference. For $\Delta m_{32}^{2}$ NO hypothesis is considered only. This figure is taken from [25].

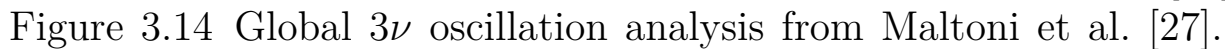
Projections of the $\chi^{2}$ function onto the parameters $\Delta m_{21}^{2}$ (solar), $\Delta m_{31}^{2} / \Delta m_{32}^{2}$ (atmospheric), $\sin ^{2} \theta_{i j}$, and $\delta$, for Normal Ordering (blue) and Inverted Ordering (red). In each panel all the undisplayed parameters are marginalized. 
Figure 4.1 a)Tree level first order Feynman diagrams representation of the $\pi^{+}$decay. b) A representation of the final excluded helicity state for the limit $m_{l}=0$. In this case one can just have a left-handed helicity neutrino and a right-handed helicity antilepton that violate the spin angular momentum conservation. The green arrows represents the particle spin and the thin black ones representing the momentum of the particles. Figure edited from [2]

Figure 4.2 Tree level Feynman diagram of the $\mu^{+}$decay where the particles 4-momenta are indicated with the letters $p, k_{1}, k_{2}$ and $k_{3}$.

Figure 4.3 The differential decay rate of the $\mu^{+}$decay at rest with respect the $E_{\nu_{e}} / m_{\mu}$ (dashed) and $E_{\bar{\nu}_{\mu}\left(e^{+}\right)} / m_{\mu}$ (solid).

Figure 4.4 Representation of the final state helicities.

Figure 4.5 Unoscillated spectrum of emitted neutrinos fro $\pi^{+}$DAR. 68

Figure 4.6 $P\left(\nu_{\mu} \rightarrow \nu_{e}\right)$ as a function of the baseline $L$ at a fixed energy $E=30 \mathrm{MeV}$ for different values of $\delta=0, \pi / 2, \pi 3 / 2$ and $\pi .69$

Figure $4.7 \mathrm{~d} N_{\bar{\nu}_{e}} / \mathrm{d} E_{\bar{\nu}_{e}}$ as a function of neutrino energy $E_{\bar{\nu}_{e}}$ for a fixed $B=30$ factor at different values of $\delta=0, \pi / 2, \pi 3 / 2$ and $\pi .71$

Figure 4.8 Numerical estimation of $\nu_{e}$ and $\bar{\nu}_{e}$ relevant interaction cross sections for different targets. Abbreviations: IBD, inverse beta decay; NC, neutral current.

Figure 4.9 Simulation of a $e^{-}$(left), $e^{+}$(right) detection. Each pixel represents an optical fiber and the colour indicates the number of photons collected by it. Appear evident the different signature that will help in the events discrimination.

Figure $4.10 \mathrm{~d} N_{\bar{\nu}_{e}} / \mathrm{d} E_{\bar{\nu}_{e}}$ (black) and its background (red).

Figure 5.1 Sensitivity plot in number of sigma respect to the true value of $\delta$. We compared the three $s_{23}^{2}$ cases [LOW,MID,HIGH] without marginalization of $s_{23}^{2}$ and $\eta$ just to appreciate the overall difference between them.

Figure 5.2 Sensitivity plot in number of sigma respect to the true value of $\delta$. We compared the shapes for the absence of marginalization (black), the marginalization of just $s_{23}^{2}$ and the marginalization of just $\eta$ parameter.

Figure 5.3 Plot of $\chi_{\nu_{e}}^{2}$ and $\left.\chi_{\bar{\nu}_{e}}^{2}\right|_{\delta^{f i t}=0, \pi}$ at fixed $s_{23}^{2}=0.7$ (solid) and $s_{23}^{2}=0.4$ (dashed).

re 5.4 Sensitivity plot in number of sigma respect to the true value of $\delta$ at different baselines at a fixed values of $\eta$.

Figure 5.5 Sensitivity plot in number of sigma respect to the true value of $\delta$ for the presence (solid) and the absence (dashed) of background for $s_{23}^{2}=[0.441,0.500,0.587]$.

Figure 5.6 Coverage of the range of $\delta$ where the $\mathrm{CP}$ violation can be established with a sensitivity equal or bigger than $5 \sigma$ with respect to $B$ parameter. I choose different values of $\eta=0.3$ (blue), 0.7 (black), 1.0 (red) with different uncertainties $\sigma_{\eta}=$ $10 \%$ (dotted dashed), $5 \%$ (solid) and $10^{-4 \%}$ (dashed). 
Figure 5.7 Coverage of the range of $\delta$ where the $\mathrm{CP}$ violation can be established with a sensitivity equal or bigger than $5-\sigma$ with respect to $\eta$ parameter for $B=30$ and $s_{23}^{2}=0.500$. We show the results for different $\sigma_{\eta}=10 \%, 5 \%, 10^{-4 \%}$. There is also represented an unreal configuration (solid orange) for $B=100$ and $\sigma_{\text {eta }}=10^{-4 \%}$ that we use as a reference of the best possible coverage.

Figure 5.8 This plot shows how to convert the value of our parameter $\eta$ in terms of loading fraction. The number of interaction has been calculated considering a $\sigma_{\nu_{e}}=50 \times \sigma_{I B D}$.

Figure 5.9 Expected significance in number of sigma to exclude $\delta=0, \pi$ for the combination $B=30$ and $\eta_{0}=0.3$ and $\sigma_{\eta}=5 \% \eta_{0}$ and for the three $s_{23}^{2}$ case (high,mid and low).

Figure 5.10 The $1 \sigma$ precision of the $\delta$ measurement as a function of the true $\delta$ value for the three $s_{23}^{2}$ cases (high, mid and low). Here we do not marginalize over $\eta$ and $s_{23}^{2}$.

Figure 5.11 The expected $1 \sigma$ and $2 \sigma$ allowed regions in the $\sin ^{2} \theta_{23}-\delta$ plane. The results for the true values of $\delta=\left(-90^{\circ}, 0,90^{\circ}\right)$ and for $s_{23}^{2}=(0.441,0.5,0.587)$ are shown.

Figure 6.1 Probabilities for $\nu_{\mu} \rightarrow \nu_{\mu}$ disappearance channel with and without the effect of invisible decay as a function of the neutrino energy are shown for $\alpha_{3}=5 \times 10^{-5} \mathrm{eV}^{2}$ for $L=295$ $\mathrm{km}$ (left panel) and $1100 \mathrm{~km}$ (right panel). The CP phase was fixed to $\delta=-\pi / 2$. The matter density of $2.6 \mathrm{~g} / \mathrm{cm}^{3}\left(3.0 \mathrm{~g} / \mathrm{cm}^{3}\right)$ were considered for $L=295 \mathrm{~km}(1100 \mathrm{~km})$.

Figure 6.2 Probabilities for $\nu_{\mu} \rightarrow \nu_{e}$ (left panels) and $\bar{\nu}_{\mu} \rightarrow \bar{\nu}_{e}$ (right panels) channel in the absence and presence of the decay effect in the upper (lower) panel for the baseline $L=295 \mathrm{~km}(1100 \mathrm{~km})$ as a function of the neutrino energy for $\alpha_{3}=5 \times 10^{-5} \mathrm{eV}^{2}$ are shown. For $L=295$ (1100) $\mathrm{km}$ the matter density of $2.6 \mathrm{~g} / \mathrm{cm}^{3}$ $\left(3.0 \mathrm{~g} / \mathrm{cm}^{3}\right)$ were used. We show the cases where $\delta= \pm \pi / 2,0$ and $\pi$.

Figure 6.3 Probabilities for $\nu_{\mu} \rightarrow \nu_{\mu}$ disappearance channel with and without invisible decay as a function of the neutrino energy are shown for $\alpha_{3}=5 \times 10^{-5} \mathrm{eV}^{2}$ for $L=295 \mathrm{~km}$ (left panel) and $1100 \mathrm{~km}$ (right panel). The CP phase was fixed to $\delta=-\pi / 2$. The matter density of $2.6 \mathrm{~g} / \mathrm{cm}^{3}\left(3.0 \mathrm{~g} / \mathrm{cm}^{3}\right)$ were considered for $L=295 \mathrm{~km}(1100 \mathrm{~km})$. The case for the visible decay indicated by the dashed blue curves were computed by using the formula defined in (6-12).

Figure 6.4 Effective probabilities for $\nu_{\mu} \rightarrow \nu_{e}$ (left panels) and $\bar{\nu}_{\mu} \rightarrow \bar{\nu}_{e}$ (right panels) defined in Eq.(6-12) as a function of the neutrino energy are shown, in the upper and lower 2 panels, respectively, for $L=295 \mathrm{~km}$ and $L=1100 \mathrm{~km}$, for $\alpha_{3}=2 \times 10^{-5} \mathrm{eV}^{2}$, and the mass ratios of $m_{3} / m_{1}=1.2,2.0$ and 100. The matter density of $2.6 \mathrm{~g} / \mathrm{cm}^{3}\left(3.0 \mathrm{~g} / \mathrm{cm}^{3}\right)$ were considered for $L=295 \mathrm{~km}(1100 \mathrm{~km})$. 
Figure 6.5 Contribution of each helicity in the visible decay, in $\Delta P_{\mu e}=P_{e f f}^{\text {osc }+ \text { decay }}\left(\nu_{\mu} \rightarrow \nu_{e} ; E_{\nu_{e}}\right)-P_{\text {inv }}\left(\nu_{\mu} \rightarrow \nu_{e} ; E_{\nu_{e}}\right)$ as a function of the neutrino energy are shown, in th upper and lower 2 panels, respectively, for $\mathrm{L}=295 \mathrm{~km}$ and $\mathrm{L}=1100$ $\mathrm{km}$, for $\alpha_{3}=2 \times 10^{-5} \mathrm{eV}^{2}$, and the mass ratios of $m_{3} / m_{1}=2.0$. The matter density of $2.6 \mathrm{~g} / \mathrm{cm}^{3}\left(3.0 \mathrm{~g} / \mathrm{cm}^{3}\right)$ were considered for $L=295 \mathrm{~km}(1100 \mathrm{~km})$.

Figure 6.6 Expected events number distribution for the disappearance channel for T2HK for $\nu_{\mu} \rightarrow \nu_{\mu}$ (left panel) and $\bar{\nu}_{\mu} \rightarrow \bar{\nu}_{\mu}$ (right panel).

Figure 6.7 Expected events number distribution for the disappearance channel for T2HK for $\nu_{\mu} \rightarrow \nu_{e}$ (left panel) and $\bar{\nu}_{\mu} \rightarrow \bar{\nu}_{e}$ (right panel) for the case of standard oscillation against invisible decay effect with $\alpha_{3}=5 \times 10^{-5} \mathrm{eV}^{2}$.

Figure 6.8 Expected events number distribution for the appearance channel for T2HK for $\nu_{\mu} \rightarrow \nu_{e}$ (left panel) and $\bar{\nu}_{\mu} \rightarrow \bar{\nu}_{e}$ (right panel) for the case of standard oscillation against invisible decay and scalar coupling helicity conserving visible decay effect. For both cases we used $\alpha_{3}=5 \times 10^{-5} \mathrm{eV}^{2}$ and for the visible case the mass ratio $m_{3} / m_{1}=2.0$.

Figure 6.9 Expected events number distribution for the disappearance channel for T2HKK for $\nu_{\mu} \rightarrow \nu_{\mu}$ (left panel) and $\bar{\nu}_{\mu} \rightarrow \bar{\nu}_{\mu}$ (right panel) for the case of standard oscillation against invisible decay effect with $\alpha_{3}=5 \times 10^{-5} \mathrm{eV}^{2}$.

Figure 6.10 Expected events number distribution for the appearance channel for T2HKK for $\nu_{\mu} \rightarrow \nu_{e}$ (left panel) and $\bar{\nu}_{\mu} \rightarrow \bar{\nu}_{e}$ (right panel) for the case of standard oscillation against invisible decay effect with $\alpha_{3}=5 \times 10^{-5} \mathrm{eV}^{2}$.

Figure 6.11 Expected events number distribution for the appearance channel for T2HK for $\nu_{\mu} \rightarrow \nu_{e}$ for the case of standard oscillation against invisible decay and scalar coupling helicity conserving visible decay effect. For both cases we used $\alpha_{3}=5 \times 10^{-5} \mathrm{eV}^{2}$ and for the visible case the mass ratio $m_{3} / m_{1}=2.0$.

Figure 6.12 Expected sensitivity obtained for the $\nu_{\mu} \rightarrow \nu_{\mu}$ channel. We can exclude with $90 \%$ C.L. at best $\alpha_{3}<8.4 \times 10^{-5} \mathrm{eV}^{2}$ for the case of a pseudo-scalar coupling in the helicity conserving case.

Figure 6.13 Expected sensitivity obtained for the $\bar{\nu}_{\mu} \rightarrow \bar{\nu}_{\mu}$ channel. We can exclude with $90 \%$ C.L. at best $\alpha_{3}<8.2 \times 10^{-5} \mathrm{eV}^{2}$ for the case of a pseudo-scalar coupling in the helicity conserving case.

Figure 6.14 Expected sensitivity obtained for the $\nu_{\mu} \rightarrow \nu_{e}$ channel. We can exclude with $90 \%$ C.L. at best $\alpha_{3}<7.0 \times 10^{-6} \mathrm{eV}^{2}$ for the case of a scalar coupling in the helicity non conserving case. 101

Figure 6.15 Expected sensitivity obtained for the $\bar{\nu}_{\mu} \rightarrow \bar{\nu}_{e}$ channel. We can exclude with $90 \%$ C.L. at best $\alpha_{3}<8.0 \times 10^{-6} \mathrm{eV}^{2}$ for the case of a pseudo-scalar coupling in the helicity non conserving case. 
Figure A.1 A thin bar bent under an external force that breaks the initial $U(1)$ symmetry of the system.

Figure A.2 The Higgs double eigenvalues respect to all the generators that fix the action of an element of $S U(2)_{L} \times U(1)_{Y}$.

Figure A.3 The potential $V(\boldsymbol{\Phi})$ has a minimum in $\boldsymbol{\Phi}^{\dagger} \boldsymbol{\Phi}=v^{2} / 2$ and a local minimum in $\boldsymbol{\Phi}^{\dagger} \boldsymbol{\Phi}=0$.

Figure B.1 The function defined in Eq.(B-4), $Q_{\mu \mu}\left(E_{\nu_{\mu}}^{0}, E_{\nu_{\mu}}\right)$ and $Q_{\mu e}\left(E_{\nu_{\mu}}^{0}, E_{e}\right)$ for four different helicity decay modes are shown, respectively, in the upper and lower panels as a function of the neutrino energy for $L=295 \mathrm{~km}$ (left panels) and $L=1100 \mathrm{~km}$ (right panels) in the presence (absence) of matter effect with $2.6 \mathrm{~g} / \mathrm{cm}^{3}\left(3.0 \mathrm{~g} / \mathrm{cm}^{3}\right)$ in the presence of the decay effect with $\alpha_{3}=5 \times 10^{-5} \mathrm{eV}^{2}$. It is shown for the cases where the energies of parent neutrino are $3.0 \mathrm{GeV}$ with and the $\mathrm{CP}$ phase is taken $\delta=-\pi / 2$.

Figure B.2 The $\Gamma_{\nu_{3}^{A} \rightarrow \nu_{1}^{B}}\left(E_{\nu_{\alpha}}, E_{\nu_{\beta}}\right)$ as a function of the daugther neutrino energy $E_{\nu_{\beta}}$. We show in the upper (lower) panels the case of scalar (pseudo-scalar) coupling for helicity conserving case (left column) and helicity non- conserving case (right column) for $\alpha_{3}=5 \times 10^{-5} \mathrm{eV}^{2}$ and a fixed parent neutrino energy $E_{\nu_{\alpha}}=3 \mathrm{GeV}$.

Figure C.1 Fitted width and shift for different values of $E_{\nu}$ and for different interaction type.

Figure C.2 The expected number of $\nu_{\mu} / \bar{\nu}_{\mu}$ events and efficiencies for each flavour and interaction type

Figure C.3 The expected number of $\nu_{e} / \bar{\nu}_{e}$ events and efficiencies for each flavour and interaction type 


\section{List of tables}

Table 2.1 The SM fermions structure.

Table 2.2 Eigenvalues of the weak isospin $I$, of its third component $I_{3}$, of the hypercharge $Y$, and of the charge $Q=I_{3}+Y / 2$ of the fermion doublets and singlets.

Table 2.3 Values of $g_{L}, g_{R}, g_{V}, g_{A}$ for the fermion fields. The superscripts $\nu, l, U, D$, indicate, respectively, a generic neutrino, charged lepton, up-type quark, and down-type quark.

Table 3.1 Reactions and decays relevant to atmospheric neutrino and accelerator neutrino production. The first column shows the index of the reaction or decay, and the second column shows the reaction or decay channel. The third column shows the branching ratio. This table is taken from [8].

Table 3.2 Key parameters of the three experiment we are referring to. Data from [25].

Table 3.3 Oscillation parameter table from Maltoni et al.[27]. $\quad 58$

Table $4.1 \mathrm{~d} N_{\bar{\nu}_{e}} / \mathrm{d} E_{\bar{\nu}_{e}}$ parameters. 


\section{1 \\ Introduction}

Neutrino physics is a really active field of research since 1930 when Pauli first proposed the existence of these particles. The attention of the whole community became even bigger when in 2015 the experiments SNO and SuperKamiokande have been awarded with the Nobel prize "for the discovery of neutrino oscillations, which shows that neutrinos have mass". This discovery open a new era for high energy physics. This was the first consistent proof that there is physics Beyond the Standard Model (BSM). Having deep knowledge about neutrino properties and phenomenology seems to be one of the most reasonable way to go to step into this unknown field. A lot of experiments have been performed and until today we collected a lot of data that have been almost all explained by the $3 \nu$ model, i.e. the model that presents just three active neutrinos. In this scenario, one of the most important unknown parameters is the leptonic $\mathrm{CP}$ violation phase $\delta$. Measuring this parameter, the neutrino mass ordering and the $\theta_{23}$ octant determination are the main challenges of the next generation experiments. In order to perform these measurements higher precision and higher statistics are required with respect to the previous generation experiments. Such high precision and high statistic measurements open up some room to also test neutrino physics beyond the $3 \nu$ model.

This work was inspired from these ideas. We first study a novel method to measure the leptonic CP violation and then we evaluate the sensitivity of Hyper-Kamiokande, the upgrade of the Super-Kamiokande experiment, to put a limit on the $\nu_{3}$ neutrino mass eigenstate lifetime. 


\section{2}

\section{Neutrinos: Who are They?}

In this chapter we are going to recall some well known neutrino properties. We will start with a brief description of the standard model of particle physics with a remark for the weak interaction to arrive to write down the Lagrangian for a neutrino field and so how it interacts. Then we will recall the basics of the oscillation properties of the neutrino flavour state with some stress on the theoretical aspects to provide a basic framework for the following chapters.

\section{1}

\section{The Standard Model}

The Standard Model (SM) of particle physics is a gauge theory based on the local symmetry group $S U(3)_{C} \times S U(2)_{L} \times U(1)_{Y}$ that accurately describes the fundamental interactions: the strong, the weak and the electromagnetic one. Indeed the subscript $C, L$ and $Y$ denote color, left-handed chirality and weak hypercharge, respectively. The number of generators of each symmetry group corresponds to the number of the vector bosons that mediate the interactions: we have $\operatorname{dim}\left(S U(3)_{C}\right)=8$ massless gluons mediating the strong force ; $\operatorname{dim}\left(S U(2)_{L}\right)=3$ massive bosons $\left(W^{ \pm}\right.$and $\left.Z\right)$ and $\operatorname{dim}\left(U(1)_{Y}\right)=1$ massless one ( $\gamma$, the photon). Due to the fact that neutrino can only interact through the weak ${ }^{1}$ force, we are interested just on the $S U(2)_{L} \times U(1)_{Y}$ sector of the gauge group. We can do this type of factorization because the $S U(3)_{C}$ symmetry is unbroken and so there is no mixing between this sector and the electroweak one.

The model as just as it stands at this level fix the interactions with only three independent unknown parameters, the coupling constants of the $S U(3)_{C}, S U(2)_{L}$ and $U(1)_{Y}$ groups, determined from the experiments. On the other hand, the number and the properties of scalar bosons and fermions are unconstrained but they must transform in a definite way under the action of the symmetry group. So the fact that in nature we have three generations of fermions is not addressed in the SM, as well as the fermions mass magnitude.

\footnotetext{
${ }^{1}$ This statement has to be intended as relative to the interactions in the SM. Neutrino can actually interact gravitationally as well.
} 
The known elementary fermions are divided into two categories, quarks and leptons, and to each categories belong two type of quarks and two types of leptons. They are distinguished by the fact that quarks participate in all the interactions, whereas charged leptons participate in all except the strong one. Each different type is also called flavour and they are arranged as is showed in Table 2.1.

\begin{tabular}{lccc}
\hline & $1^{\text {st }}$ generation & $2^{\text {nd }}$ generation & $3^{\text {rd }}$ generation \\
\hline quarks: & $u$ (up), & $c$ (charm), & $t$ (top), \\
& $d$ (down), & $s$ (strange), & $b$ (bottom); \\
leptons: & $\nu_{e}$ (electron neutrino), & $\nu_{\mu}$ (muon neutrino), & $\nu_{\tau}$ (tau neutrino), \\
& $e$ (electron), & $\mu$ (muon), & $\tau$ (tau). \\
\hline
\end{tabular}

Table 2.1: The SM fermions structure.

The fermion sector of the SM depends on 13 independent parameters: six quarks masses, three charged lepton masses (neutrinos are assumed to be massless in the SM), three quarks mixing angles and one phase. The values of all these parameters must be determined from experimental measurements. 


\section{2}

\section{The Electroweak Interaction}

In order to introduce the electroweak Lagrangian let us recall some properties of fermions and some relativistic formalism. First of all a fermion (so a particle with spin 1/2) is well represented by a Dirac quantized field, i.e. an irreducible representation of the Lorentz group extended by parity $(S U(2) \times S U(2))$, a 4 -spinor

$$
\psi=\left(\begin{array}{l}
u_{1} \\
u_{2} \\
v_{1} \\
v_{2}
\end{array}\right)=\left(\begin{array}{l}
u \\
v
\end{array}\right),
$$

where each couple of components is a representation of an $S U(2)$ group. Quarks and massive leptons are described by massive Dirac fields whereas neutrinos are described by massless Dirac fields in the SM. A spinor must satisfy the Dirac equation

$$
(\mathrm{i} \not \partial-m) \psi=0,
$$

together with the Klein-Gordon equation

$$
\left(\square+m^{2}\right) \psi=0,
$$

where $\square \equiv \partial_{\mu} \partial^{\mu}, \not \partial=\gamma^{\mu} \partial_{\mu}$ and $\partial^{\mu}=\left(\partial_{t},-\vec{\partial}\right)$ with $\mu=0,1,2,3$ indicating the 4 space-time components. The $\gamma^{\mu}$ are a set of $4 \times 4$ matrices called Dirac matrices that satisfies the anticommutation relations

$$
\left\{\gamma^{\mu}, \gamma^{\nu}\right\}=2 g^{\mu \nu}, \quad \gamma^{0} \gamma^{\mu \dagger} \gamma^{0}=\gamma^{\mu},
$$

and $g^{\mu \nu}=\operatorname{diag}(1,-1,-1,-1)$.

For our specific purpose it is convenient to choose what is called the Chiral representation of the $\gamma^{\mu}$ (each matrix element is to be considered a $2 \times 2$ matrix)

$$
\gamma^{0}=\left(\begin{array}{cc}
0 & -1 \\
-1 & 0
\end{array}\right), \quad \gamma^{i}=\left(\begin{array}{cc}
0 & \sigma^{i} \\
-\sigma^{i} & 0
\end{array}\right), \quad \gamma^{5}=\mathrm{i} \gamma^{0} \gamma^{1} \gamma^{2} \gamma^{3}=\left(\begin{array}{cc}
1 & 0 \\
0 & -1
\end{array}\right)
$$

with $\sigma^{i}$ being the Pauli matrices and $\gamma^{5}$ is the chirality matrix. This chirality matrix is useful to define what are called the chiral projectors

$$
\psi=\psi_{R}+\psi_{L}=R \psi+L \psi=\left(\begin{array}{c}
\psi_{R} \\
\psi_{L}
\end{array}\right), \quad L=\frac{1}{2}\left(1-\gamma^{5}\right) \quad R=\frac{1}{2}\left(1+\gamma^{5}\right),
$$

and for the adjoint spinor

$$
\bar{\psi}=\psi^{\dagger} \gamma^{0}=\bar{\psi}_{R}+\bar{\psi}_{L}=\bar{\psi} L+\bar{\psi} R=\left(\begin{array}{ll}
\overline{\psi_{L}} & \overline{\psi_{R}}
\end{array}\right) .
$$


Another important covariant quantity that characterizes fermions is the helicity

$$
\hat{h}=\frac{\Sigma \cdot \vec{P}}{|\vec{P}|} \quad \Sigma_{k}=\gamma_{0} \gamma_{k} \gamma_{5},
$$

with $\Sigma$ being the spin operator and $\vec{P}$ is the momentum operator. This operator has eigenvalues \pm 1 because $\hat{h}^{2}=1$. From a physical point of view this quantity represents the projection of the spin of a particle along its momentum direction. With this notation in mind we can notice that the scalar fermion term (the mass term) mixes right and left-handed components

$$
\bar{\psi} \psi=\bar{\psi}_{R} \psi_{L}+\bar{\psi}_{L} \psi_{R}
$$

On the other hand the vector current does not do that

$$
\bar{\psi} \gamma^{\mu} \psi=\bar{\psi}_{R} \gamma^{\mu} \psi_{R}+\bar{\psi}_{L} \gamma^{\mu} \psi_{L}
$$

The second term of this equation can be rewrite in the form

$$
\bar{\psi}_{L} \gamma^{\mu} \psi_{L}=\bar{\psi} R \gamma^{\mu} L \psi=\bar{\psi} \gamma^{\mu} L^{2} \psi=\bar{\psi} \gamma^{\mu} L \psi=\frac{1}{2} \bar{\psi}\left(1-\gamma^{5}\right) \psi
$$

that is the well known $\mathrm{V}$-A current (vector-axial).

Let us now build the electroweak part of the Lagrangian. First of all let us write down the (2-10) for the first generation of leptons, i.e. the electron and the electron neutrino we have

$$
\mathscr{L}=\mathrm{i} \overline{e_{R}} \not \partial e_{R}+\mathrm{i} \overline{e_{L}} \not \partial e_{L}+\mathrm{i} \overline{\nu_{e L}} \not \partial \nu_{e_{L}} .
$$

It is really important to note that we did not write the right-handed chiral neutrino part (or the left-handed chiral antineutrino part) because there is no experimental evidence of its existence. So the SM does not (in principle) allow neutrinos to have mass through the Higgs mechanism that we will just cite later on. From these terms we can deduce what is the internal symmetry by introducing left-isospin and the right-singlet

$$
\mathrm{L}=\left(\begin{array}{c}
\nu_{e L} \\
e_{L}
\end{array}\right), \quad \mathrm{R}=e_{R},
$$

so that $\mathrm{L}$ is a isospin doublet in this representation of $S U(2)_{L}$ with isospin third component $I_{3}= \pm 1 / 2$ and $\mathrm{R}$ is a singlet with $I_{3}=0$. Using the convention (2-13) we can rewrite the Lagrangian in a very elegant and useful way

$$
\mathscr{L}=\mathrm{i} \overline{\mathrm{R}} \not \partial \mathrm{R}+\mathrm{i} \overline{\mathrm{L}} \not \partial \mathrm{L} .
$$

In this $2 \times 2$ representation the generators of the algebra of the $S U(2)_{L}$ group are simply the three Pauli matrices that satisfy the commutation relation 


$$
\left[I_{a}, I_{b}\right]=\mathrm{i} \epsilon_{a b c} I_{c}, \quad I_{a}=\frac{\sigma_{a}}{2}, \text { with } a=1,2,3,
$$

where $\epsilon_{a b c}$ is the Levi Civita antisymmetric tensor that assumes the values \pm 1 if the indices $a b c$ are different from one another and are dispose in a even (odd) permutation, and it is 0 when some of the indices are the same. The other symmetry of this Lagrangian is the $U(1)_{Y}$ one generator to this algebra and we obtain

$$
\left[I_{a}, Y\right]=0, \quad Q=I_{3}+\frac{Y}{2},
$$

where the second condition is the Gell-Mann-Nishijima relation that connects the hypercharge to the electromagnetic charge. From this last relation it is easy to see that $\mathrm{L}$ has hypercharge -1 and $\mathrm{R}$ has hypercharge -2 . Now we have fixed the action of $U(1)_{Y}$ group on the fields

$$
U(1)_{Y}:\left(\begin{array}{c}
\nu_{e L} \\
e_{L} \\
e_{R}
\end{array}\right) \rightarrow\left(\begin{array}{ccc}
e^{\mathrm{i} \theta / 2} & 0 & 0 \\
0 & e^{\mathrm{i} \theta / 2} & 0 \\
0 & 0 & e^{\mathrm{i} \theta}
\end{array}\right)\left(\begin{array}{c}
\nu_{e L} \\
e_{L} \\
e_{R}
\end{array}\right),
$$

and the action of $S U(2)_{L}$ as well

$$
S U(2)_{L}:\left(\begin{array}{c}
\nu_{e L} \\
e_{L} \\
e_{R}
\end{array}\right) \rightarrow\left(\begin{array}{cc|c}
e^{-(\mathrm{i} / 2) \vec{\sigma} \cdot \vec{\theta}} & & 0 \\
& & 0 \\
\hline 0 & 0 & 1
\end{array}\right)\left(\begin{array}{c}
\nu_{e L} \\
e_{L} \\
e_{R}
\end{array}\right) .
$$

Now that we have written down the formalism for the free Lagrangian we will introduce gauge fields to take into account the interactions between the particles.

We are not going into the details of gauge theories, so, the shortcut to do this is to consider a couple of canonical prescription. First, associate to each generator of the group a gauge field

$$
\begin{aligned}
S U(2)_{L} & \rightarrow W_{\mu}^{1}, W_{\mu}^{2}, W_{\mu}^{3} \equiv \vec{W}_{\mu} \\
U(1)_{Y} & \rightarrow B_{\mu} .
\end{aligned}
$$

Second, in order to have local gauge invariance we have to introduce a covariant derivative $D_{\mu}$ that will replace the ordinary one

$$
D_{\mu} \rightarrow \partial_{\mu}+\mathrm{i} g \frac{\vec{\sigma}}{2} \cdot \vec{W}_{\mu}+\mathrm{i} g^{\prime} B_{\mu} \frac{Y}{2},
$$

where $g$ and $g^{\prime}$ are the coupling constants associated to the groups $S U(2)_{L}$ and $U(1)_{Y}$, respectively. Applying these prescription to the first generation lepton Lagrangian (2-14) one obtains

$$
\mathscr{L}_{\text {gauge }}=\mathscr{L}+\mathrm{i} \overline{\mathrm{L}} \gamma^{\mu}\left(g \frac{\vec{\sigma}}{2} \cdot \vec{W}_{\mu}+\mathrm{i} g^{\prime} B_{\mu} \frac{Y}{2}\right) \mathrm{L}+\mathrm{i} \overline{\mathrm{R}} \gamma^{\mu}\left(g^{\prime} B_{\mu} \frac{Y}{2}\right) \mathrm{R} .
$$


Just from this piece of the Lagrangian we are already able to define the interaction terms, aim of this chapter. By opening up the $\mathrm{L}$ term one obtains $\mathrm{i} \overline{\mathrm{L}} \gamma^{\mu}\left(g \frac{\vec{\sigma}}{2} \cdot \vec{W}_{\mu}+\mathrm{i} g^{\prime} B_{\mu} \frac{Y}{2}\right) \mathrm{L}=-g \overline{\mathrm{L}} \gamma^{\mu}\left(\frac{\sigma^{1}}{2} W_{\mu}^{1}+\frac{\sigma^{2}}{2} W_{\mu}^{2}\right) \mathrm{L}-\overline{\mathrm{L}} \gamma^{\mu} \frac{\sigma^{3}}{2} \mathrm{~L} W_{\mu}^{3}-\frac{g^{\prime}}{2} Y \overline{\mathrm{L}} \gamma^{\mu} \mathrm{L} B_{\mu}$

The first term is charged because it connects the charged lepton with uncharged the neutrino and can be written

$$
\mathscr{L}_{\text {gauge }}^{ \pm}=-g \overline{\mathrm{L}} \gamma^{\mu}\left(\frac{\sigma^{1}}{2} W_{\mu}^{1}+\frac{\sigma^{2}}{2} W_{\mu}^{2}\right) \mathrm{L}=\frac{g}{2} \overline{\mathrm{L}} \gamma^{\mu}\left(\begin{array}{cc}
0 & W_{\mu}^{1}-\mathrm{i} W_{\mu}^{2} \\
W_{\mu}^{1}+\mathrm{i} W_{\mu}^{2} & 0
\end{array}\right) L .
$$

This expression suggests the definition of the physical interaction field $W_{\mu}^{ \pm}$

$$
W_{\mu}^{ \pm}=\frac{1}{\sqrt{2}}\left(W_{\mu}^{1} \mp \mathrm{i} W_{\mu}^{2}\right)
$$

in such a way that

$$
\begin{aligned}
\mathscr{L}_{\text {gauge }}^{ \pm} & =-\frac{g}{2 \sqrt{2}}\left[\bar{\nu}_{e} \gamma^{\mu}\left(1-\gamma^{5}\right) e W_{\mu}^{+}+\bar{e} \gamma^{\mu}\left(1-\gamma^{5}\right) \nu_{e} W_{\mu}^{-}\right]= \\
& =\frac{g}{2 \sqrt{2}}\left(j_{W, L}^{\mu} W_{\mu}^{+}+j_{W, L}^{\mu}{ }^{\dagger} W_{\mu}^{-}\right)
\end{aligned}
$$

where $j_{W, L}^{\mu}$ are called leptonic charged current and the $g$ coupling is related to the Fermi constant $G_{F}$ by the relation

$$
g=2 \sqrt{2}\left(\frac{M_{W}^{2} G_{F}}{\sqrt{2}}\right)^{1 / 2} .
$$

The interaction Lagrangian (2-25) generates the trilinear couplings, i.e. charged current interaction, represented by the diagrams in Fig.2.1.
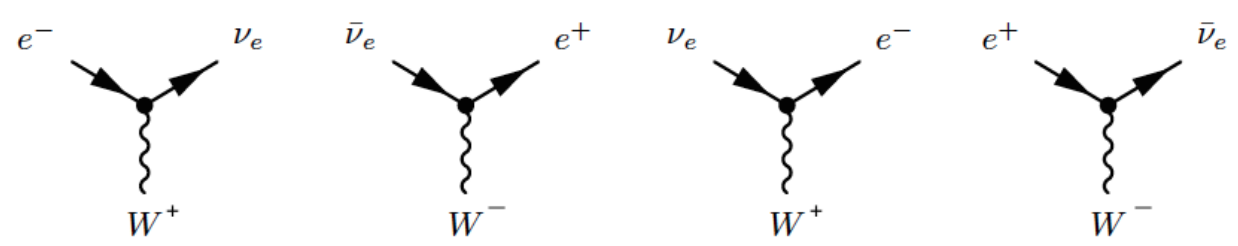

Figure 2.1: Feynman diagrams representation of the charged current interaction vertex of the first lepton generation.

The other terms of (2-21) describe the neutral current interaction

$$
\begin{aligned}
\mathscr{L}_{\text {gauge }}^{0} & =-\frac{g}{2} \overline{\mathrm{L}} \gamma^{\mu} \sigma^{3} \mathrm{~L} W_{\mu}^{3}-\frac{g^{\prime}}{2}\left(\overline{\mathrm{L}} \gamma^{\mu} Y \mathrm{~L}+\overline{\mathrm{R}} \gamma^{\mu} Y \mathrm{R}\right) \\
& =-\frac{g}{2}\left(\nu_{e}^{-} \gamma^{\mu} \nu_{e L}-\overline{e_{L}} \gamma^{\mu} e_{L}\right) W_{\mu}^{3} \\
& +\frac{g^{\prime}}{2}\left(\overline{\nu_{L}} \gamma^{\mu} \nu_{e L}+\overline{e_{L}} \gamma^{\mu} e_{L}+2 \overline{e_{R}} \gamma^{\mu} e_{R}\right) B_{\mu} .
\end{aligned}
$$

Expressing in an appropriate way the fields $B_{\mu}$ and $W_{\mu}^{3}$ one must recover 
the electromagnetic interaction of the electron and the neutral current weak interaction, $A_{\mu}$ and $Z_{\mu}$. Performing a rotation in the plane of the $B_{\mu}$ and $W_{\mu}^{3}$ fields through an angle $\theta_{W}$, the famous Weinberg angle one gets

$$
\begin{aligned}
B_{\mu} & =\cos \theta_{W} A_{\mu}-\sin \theta_{W} Z_{\mu}=c_{W} A_{\mu}-s_{W} Z_{\mu} \\
W_{\mu}^{3} & =\sin \theta_{W} A_{\mu}+\cos \theta_{W} Z_{\mu}=s_{W} A_{\mu}-c_{W} Z_{\mu}
\end{aligned}
$$

Inserting the new fields (2-28) into (2-31) we obtain

$$
\begin{aligned}
\mathscr{L}_{\text {gauge }}^{0}= & -\frac{1}{2}\left\{\bar{\nu}_{e L}\left[\left(g c_{W}+g^{\prime} s_{W}\right) \not Z_{\mu}+\left(g s_{W}-g^{\prime} c_{W}\right) / A_{\mu}\right] \nu_{e L}\right. \\
& -\bar{e}_{L}\left[\left(g c_{W}-g^{\prime} s_{W}\right) \not Z_{\mu}+\left(g s_{W}+g^{\prime} c_{W}\right) / A_{\mu}\right] e_{L} \\
& \left.-2 g^{\prime} \bar{e}_{R}\left[c_{W} / A_{\mu}-s_{W} / Z_{\mu}\right] e_{R}\right\} .
\end{aligned}
$$

Due to the fact that neutrinos are neutral particle, we deduce from the first line of (2-29) that $g s_{W}=g^{\prime} c_{W}$ in order to cancel the electromagnetic interaction term. Knowing this and with a little bit of algebra we have

$\mathscr{L}_{\text {gauge }}^{0}=-\frac{g}{2 c_{W}}\left\{\bar{\nu}_{e L} / Z_{\mu} \nu_{e L}-\left(1-2 s_{W}^{2}\right) \bar{e}_{L} / Z_{\mu} e_{L}+2 s_{W}^{2} \bar{e}_{R} / Z_{\mu} e_{R}+g s_{W} \bar{e} / A_{\mu} e\right\}$.

The last term of this equation, since it represents the electromagnetic interaction of the electron, is coupled by the electric charge so that $g s_{W}=e$. As we have seen for the charged current part, this interaction Lagrangian generates the trilinear coupling mediated by the $Z_{\mu}$ boson and the $A_{\mu}$ i.e. the photon represented by the diagrams in Fig.2.2.

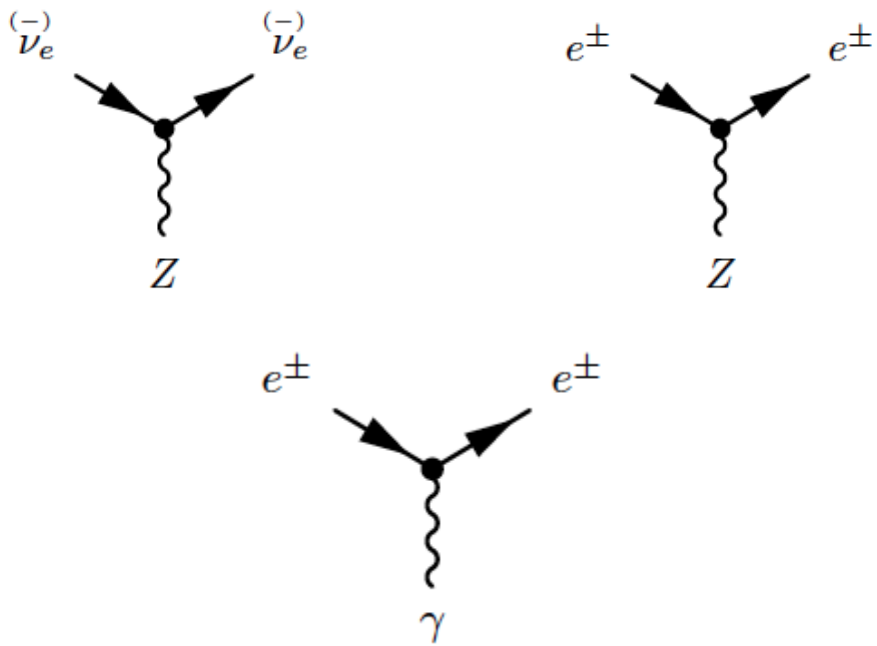

Figure 2.2: Feynman diagrams representation of the neutral current and electromagnetic interaction vertex of the first lepton generation.

The leptonic weak neutral-current Lagrangian (2-30) has another smart way to be written and it will be essential to easily write down the other 
fermion's terms. Let us just introduce the coefficients $g_{L}^{f}$ and $g_{R}^{f}$ (where the superscript $f$ indicates a charged/neutral fermion flavour ) so that

$$
\mathscr{L}_{\text {gauge }}^{0}=-\frac{g}{2 c_{W}}\left\{2 g_{L}^{\nu} \bar{\nu}_{L} / Z_{\mu} \nu_{L}+2 g_{L}^{f} \bar{f}_{L} / Z_{\mu} f_{L}+2 g_{L}^{f} \bar{f}_{R} / Z_{\mu} f_{R}+g s_{W} \bar{e} / A_{\mu} e\right\} .
$$

The general form for these new coefficients is simple, and relate the Weinberg angle and the value of the third component of the weak isospin as follows

$$
g_{L}^{f}=I_{3}^{f}-q^{f} s_{W}^{2}, \quad g_{R}^{f}=-q^{f} s_{W}^{2},
$$

where $q^{f}$ is the electromagnetic charge of the fermion and both of them can be combined together

$$
g_{V}^{f}=g_{L}^{f}+g_{R}^{f}=I_{3}^{f}-2 q^{f} s_{W}^{2}, \quad g_{A}^{f}=g_{L}^{f}-g_{R}^{f}=I_{3}^{f},
$$

to have the final version of the weak neutral-current interaction

$$
\begin{aligned}
\mathscr{L}_{\text {gauge }}^{N C} & =-\frac{g}{2 c_{W}}\left\{\overline{\nu_{e}} \gamma^{\mu}\left(g_{V}^{\nu}-g_{A}^{\nu} \gamma^{5}\right) \nu_{e}+\bar{e} \gamma^{\mu}\left(g_{V}^{l}-g_{A}^{l} \gamma^{5}\right) e\right\} Z_{\mu} \\
& =-\frac{g}{2 c_{W}} j_{W, L}^{\mu} Z_{\mu} .
\end{aligned}
$$

The first generation's total Lagrangian, of course, contains the quark terms, that one obtains simply defining the isospin doublet and right handed singlet

$$
Q=\left(\begin{array}{c}
u_{L} \\
d_{l}
\end{array}\right), \quad \mathrm{Q}_{R}=u_{R}, d_{R} .
$$

Clearly the quarks have different charges with respect to the electron and the neutrinos so the couplings associated with the symmetry group will be different, as is reported in Tables 2.2 and 2.3. In the end to get a complete Lagrangian for the electroweak interaction one must add the gauge fields kinetic term and terms coupled with the Higgs boson. 


\begin{tabular}{cccccc}
\hline & & $I$ & $I_{3}$ & $Y$ & $Q$ \\
\cline { 3 - 6 } lepton doublet & $L_{L} \equiv\left(\begin{array}{c}\nu_{e L} \\
e_{L}\end{array}\right)$ & $1 / 2$ & $1 / 2$ & -1 & 0 \\
lepton singlet & $e_{R}$ & 0 & 0 & -2 & -1 \\
quark doublet & $Q_{L} \equiv\left(\begin{array}{c}u_{L} \\
d_{L}\end{array}\right)$ & $1 / 2$ & $1 / 2$ & & $-1 / 3$ \\
quark singlets & $u_{R}$ & 0 & 0 & $4 / 3$ & $2 / 3$ \\
& $d_{R}$ & & & $-2 / 3$ & $-1 / 3$ \\
\hline
\end{tabular}

Table 2.2: Eigenvalues of the weak isospin $I$, of its third component $I_{3}$, of the hypercharge $Y$, and of the charge $Q=I_{3}+Y / 2$ of the fermion doublets and singlets.

\begin{tabular}{ccccc}
\hline Fermions & $g_{L}$ & $g_{R}$ & $g_{V}$ & $g_{A}$ \\
\hline$\nu_{e}, \nu_{\mu}, \nu_{\tau}$ & $g_{L}^{\nu}=\frac{1}{2}$ & $g_{R}^{\nu}=0$ & $g_{V}^{\nu}=\frac{1}{2}$ & $g_{A}^{\nu}=\frac{1}{2}$ \\
$e, \mu, \tau$ & $g_{L}^{l}=-\frac{1}{2}+s_{W}^{2}$ & $g_{R}^{l}=s_{W}^{2}$ & $g_{V}^{l}=-\frac{1}{2}+2 s_{W}^{2}$ & $g_{A}^{l}=-\frac{1}{2}$ \\
$u, c, t$ & $g_{L}^{U}=\frac{1}{2}-\frac{2}{3} s_{W}^{2}$ & $g_{R}^{U}=-\frac{2}{3} s_{W}^{2}$ & $g_{V}^{U}=\frac{1}{2}-\frac{4}{3} s_{W}^{2}$ & $g_{A}^{U}=\frac{1}{2}$ \\
$d, s, b$ & $g_{L}^{D}=-\frac{1}{2}+\frac{1}{3} s_{W}^{2}$ & $g_{R}^{D}=\frac{1}{3} s_{W}^{2}$ & $g_{V}^{D}=-\frac{1}{2}+\frac{2}{3} s_{W}^{2}$ & $g_{A}^{D}=-\frac{1}{2}$ \\
\hline
\end{tabular}

Table 2.3: Values of $g_{L}, g_{R}, g_{V}, g_{A}$ for the fermion fields. The superscripts $\nu, l, U, D$, indicate, respectively, a generic neutrino, charged lepton, up-type quark, and down-type quark. 


\section{3}

\section{Neutrino Interactions: The Inverse Beta Decay}

Even though the SM is not able to describe the fact that neutrino has mass and its oscillation phenomenology, it describes with really high accuracy neutrino's interactions. As we have seen in the previous chapter we have two kind of electroweak interactions: the charged current $(\mathrm{CC})$ and the neutral current (NC). These two interactions are mediated by the $W^{ \pm}$and $Z$ bosons respectively. As a consequence of a process that is called spontaneous symmetry breaking these bosons acquire mass by the Higgs mechanism (see Appendix A). Because of their massive nature they behave in a different way with respect to their massless counterpart, i.e. the photon $A_{\mu}$. Indeed if we write down the 2-points propagator for the photon

$$
G_{\mu \nu}\left(x-x^{\prime}\right)=\lim _{\epsilon \rightarrow 0} \mathrm{i} \int \frac{\mathrm{d}^{4} p}{(2 \pi)^{4}} \frac{-g_{\mu \nu}}{p^{2}-\mathrm{i} \epsilon} e^{-\mathrm{i} p \cdot\left(x-x^{\prime}\right)},
$$

we can see that at the denominator just the photon's momentum appears. In the $W^{ \pm}$and $Z$ case, because they have a rest mass, the propagators become

$$
\begin{aligned}
G_{\mu \nu}^{(W)}\left(x-x^{\prime}\right) & =\lim _{\epsilon \rightarrow 0} \mathrm{i} \int \frac{\mathrm{d}^{4} p}{(2 \pi)^{4}} \frac{-g_{\mu \nu}+\frac{p_{\mu} p_{\nu}}{m_{W}^{2}}}{p^{2}-m_{W}^{2}+\mathrm{i} \epsilon} e^{-\mathrm{i} p \cdot\left(x-x^{\prime}\right)}, \\
G_{\mu \nu}^{(Z)}\left(x-x^{\prime}\right) & =\lim _{\epsilon \rightarrow 0} \mathrm{i} \int \frac{\mathrm{d}^{4} p}{(2 \pi)^{4}} \frac{-g_{\mu \nu}+\frac{p_{\mu} p_{\nu}}{m_{Z}^{2}}}{p^{2}-m_{Z}^{2}+\mathrm{i} \epsilon} e^{-\mathrm{i} p \cdot\left(x-x^{\prime}\right)} .
\end{aligned}
$$

Because of the presence of the rest mass term, when the momentum carried by the boson is low enough it is possible to use an approximate version of these propagators. The terminology "low enough" makes sense when we remember the value of the $W$ and $Z$ bosons masses that are respectively $\sim 80.4 \mathrm{GeV}$ and $\sim 91.2 \mathrm{GeV}$ [1]. So in processes that involve energy of the order of hundreds of $\mathrm{MeV}$, like is the case we will discuss in the later chapters, we can consider the approximation

$$
G_{\mu \nu}^{(W)}(p) \stackrel{|p|^{2} \ll m_{W}^{2}}{\longrightarrow} \mathrm{i} \frac{g_{\mu \nu}}{m_{W}^{2}} \quad, \quad G_{\mu \nu}^{(Z)} \stackrel{|p|^{2} \ll m_{Z}^{2}}{\longrightarrow} \mathrm{i} \frac{g_{\mu \nu}}{m_{Z}^{2}} .
$$

This mathematical relation can be visualized like the shrinking of a Feynman diagram as we can see in Fig.2.3 so that the respective effective Lagrangians become

$$
\mathscr{L}_{\text {eff }}^{(C C)}=-\frac{G_{F}}{\sqrt{2}} j_{W \mu}^{\dagger} j_{W}^{\mu} \quad, \quad \mathscr{L}_{\text {eff }}^{(N C)}=-\frac{G_{F}}{\sqrt{2}} j_{Z \mu}^{\dagger} j_{Z}^{\mu}
$$



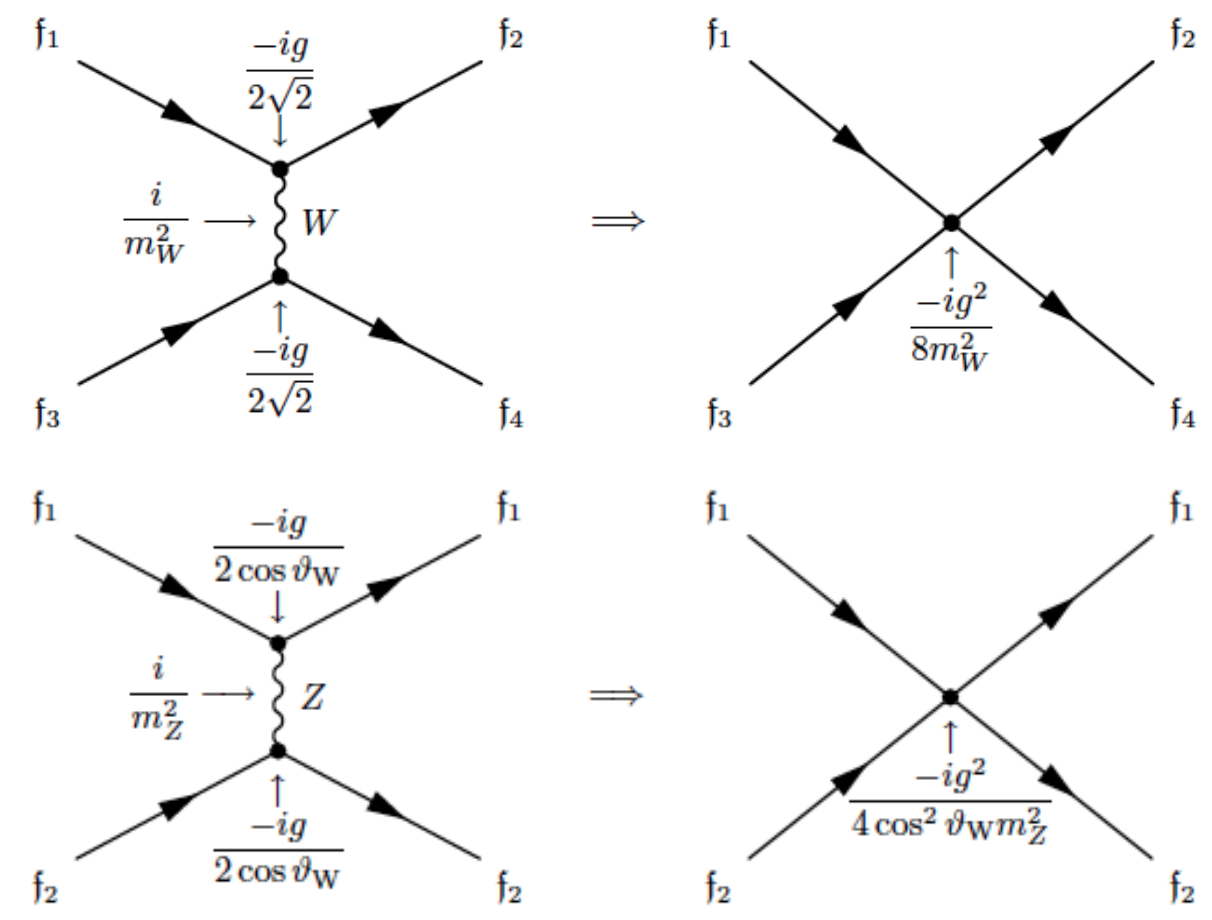

Figure 2.3: Contraction of the $W$ and $Z$ gauge boson propagator in a generic low-energy CC process. Figure from [2]

One of the most useful neutrino interaction is called inverse beta decay (IBD) that is e quasielastic scattering between a $\bar{\nu}_{e}$ and a proton $p$

$$
\overline{\nu_{e}}+p \rightarrow e^{+}+n
$$

where $e^{+}$and $n$ indicate, respectively, the positron and the neutron. This interaction have a relevant role in the study of $\bar{\nu}_{e}$ for several reasons:

1) Its cross section is relatively large with respect to the other weak processes $\sim G_{F}^{2} E_{\nu}^{2}$,

2) It can be calculated accurately,

3) It has a low threshold $E_{\nu}>1.806 \mathrm{MeV} \approx m_{e}+m_{n}-m_{p}$,

4) The positron energy is strongly correlated with the $\bar{\nu}_{e}$ energy. This point is crucial because what is actually measured is the energy of the product of the interaction, i.e. the positron energy, and so this correlation makes possible a really good reconstruction of the original $\bar{\nu}_{e}$ energy.

5) Materials that possess free proton are really cheap (e.g. water, hydrocarbon) and this permit to construct large detectors.

The complete calculation is a really long and hard one so we will just outline the crucial formulas and the final result.

First of all let us just remember how a differential cross section is evaluated: 
if we start from a initial state of two particles $a$ and $b$ and we end up with an unknown number $N_{f}$ of particles in the final state we have

$$
\frac{\mathrm{d} \sigma}{\mathrm{d} t}=S \sum_{\text {spin }}^{-} \frac{(2 \pi)^{4} \delta^{4}\left(P_{i}-P_{f}\right)|\mathcal{M}|^{2}}{4 \sqrt{\left(p_{a} \cdot p_{b}\right)^{2}-m_{a}^{2} m_{b}^{2}}} \prod_{f=1}^{N_{f}} \frac{\mathrm{d}^{3} p f}{(2 \pi)^{3} 2 E_{f}},
$$

where $p_{a}, p_{b}$ are the four-momenta of the two initial particles, $m_{a}, m_{b}$ are their rest masses, $p_{f}$ is the four-momenta of the $f^{t h}$ particle, $P_{i}=p_{a}+p_{b}$ and $P_{f}=\sum_{f=1}^{N_{f}} p_{f}$ are, respectively, the total four-momenta of the initial and final states, $\mathcal{M}$ is the total amplitude of the process, and the symbol $\sum_{\text {spin }}^{-}$ indicates an average over the unobserved spin states of the initial particles and a sum over the unobserved spin states of the final particles. $S$ is a statistical factor given by the product of a factor $1 / n$ ! for each set of $\mathrm{n}$ identical particles. The amplitudes $\mathcal{M}$ is the sum of the amplitudes of the Feynman diagrams contributing to the process, and to do so one has to follow the so called Feynman rules that translate the graphs into integrals (see [2]).

For our IBD interaction the relative tree-level (i.e. the dominant) Feynman diagram looks like Fig. 2.4. As one can see what really happen is that it is not the proton that interact with the $\bar{\nu}_{e}$ but its quark $u$. Due to the fact that $u$ is bounded to the other quarks by the strong interaction it is not possible to treat it like a free particle. So the quark current must be replaced by the appropriate hadronic transition matrix element which takes into account the incalculable effect of strong interactions [2]

$$
\bar{d} \gamma^{\mu}\left(1-\gamma^{5}\right) u \rightarrow\left\langle p\left|h^{\mu}\right| n\right\rangle
$$

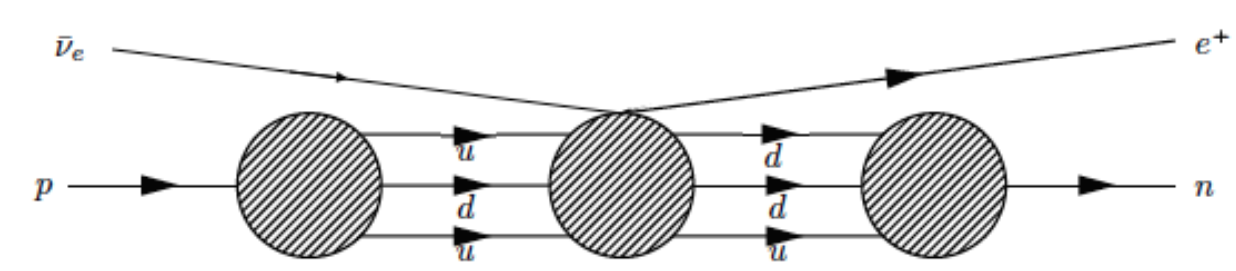

Figure 2.4: Tree-level Feynman diagram of the inverse neutron decay process in (2-40), with the neutron and proton represented as a bound states of valence quarks. The blobs represent strong interaction binding. Figure edited from [2].

In general, the hadronic matrix element must be a linear combination of a vector and an axial-vector, which can only be constructed from the available kinematical quantities. These are the neutron and proton four-momenta $p_{n}$ and $p_{p}$. So we have to substitute to this matrix element a generic term that 
takes into account all the possible weak interaction terms so that

$$
\mathcal{M}=\bar{\nu}_{e} \gamma^{\mu}\left(1-\gamma^{5}\right) e \cdot \bar{n}\left(f_{1} \gamma_{\mu}+g_{1} \gamma_{\mu} \gamma^{5}+\mathrm{i} f_{2} \sigma_{\mu \nu} \frac{q^{\nu}}{2 M}+g_{2} \frac{q_{\mu}}{M} \gamma^{5}\right) p
$$

where $\sigma_{\mu \nu}=\mathrm{i}\left[\gamma_{\mu}, \gamma_{\nu}\right] / 2, M=\left(m_{n}+m_{p}\right) / 2 \approx 938.9 \mathrm{MeV}, f_{i}, g_{i}$ are function of $q=p_{\bar{\nu}_{e}}-p_{p}$ i.e. the transferred momentum, and these functions are called formfactors. The differential cross section is [3]

$$
\frac{\mathrm{d} \sigma}{\mathrm{d} q}=\frac{G_{F}^{2} \cos ^{2} \theta_{C}}{2 \pi\left(s-m_{p}^{2}\right)}|\mathcal{M}|^{2}
$$

where $s=\left(p_{\bar{\nu}_{e}}+p_{p}\right)^{2}$.

The complete formula for the total cross section in terms of electron and $\bar{\nu}_{e}$ energy is really complicated, but we will use an approximate form that is valid for neutrinos with energies $\lesssim 300 \mathrm{MeV}[3]$

$$
\sigma_{I B D}\left(E_{\nu}\right)=\sigma_{0} p_{e} E_{e} E_{\nu}^{-0.07056+0.02018 \log E_{\nu}-0.001953 \log ^{3} E_{\nu}}
$$

where $\sigma_{0}=10^{-43} \mathrm{~cm}^{2}, p_{e}=\sqrt{E_{e}^{2}-m_{e}^{2}}$ and $E_{e}=E_{\nu}-\left(m_{n}-m_{p}\right)$. In Fig.2.5 we show this function in a range of energy that will be interesting for the argument of the second Chapter.

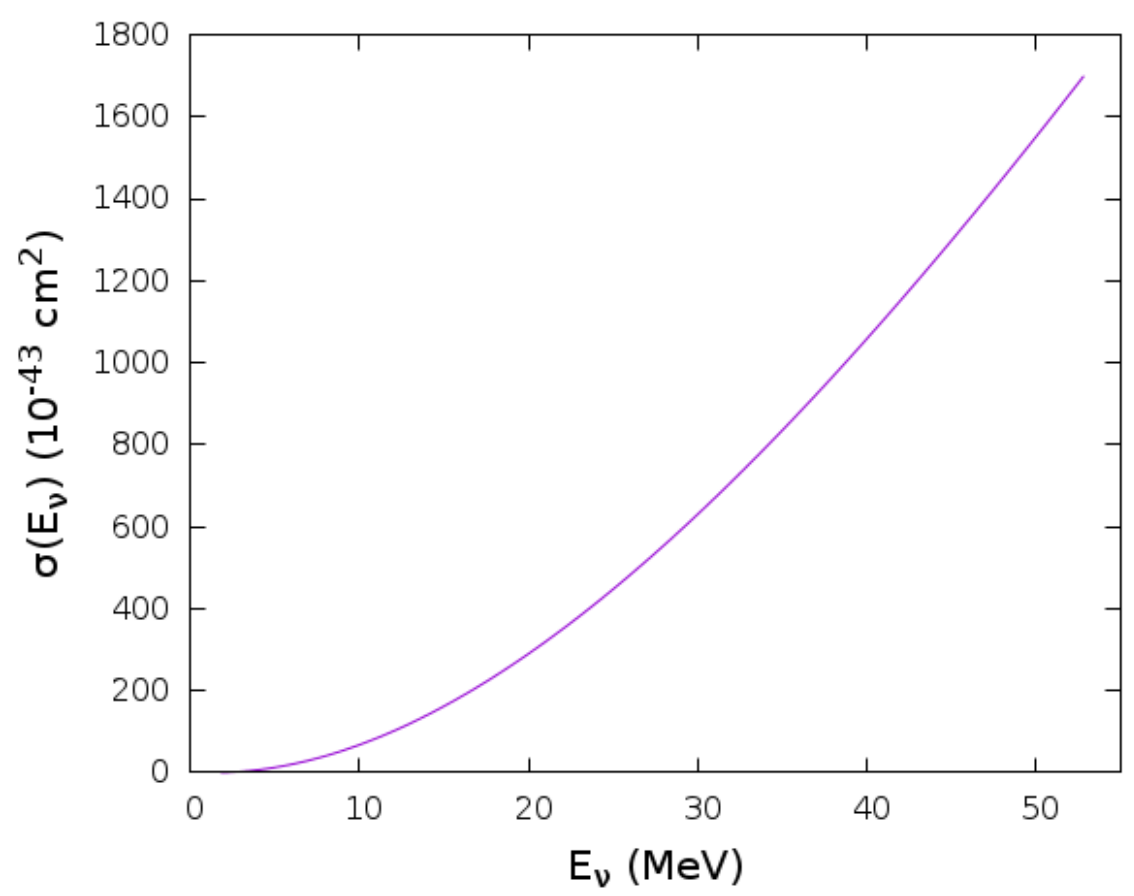

Figure 2.5: A plot of the function (2-45) in the range of $E_{\nu}=[0,52.8] \mathrm{MeV}$. 


\section{4}

\section{Neutrino Oscillation in Vacuum}

Indications that neutrino physics was deeper than what SM was predicting existed since the late 1960s when Davis et al. [4] measured a solar neutrino deficiency with respect to the flux predicted by Bahcall et al. [5].

Just recently (2002) this deficiency was confirmed to be an actual oscillation phenomena by the SNO experiment [6] that have found a $5 \sigma$ evidence for $\nu_{e} \rightarrow \nu_{\mu, \tau}$ conversion from ${ }^{8} B$ solar neutrino flux. This anomaly was confirmed 1 year later by the KamLAND collaboration [7] discovering disappearance of $\bar{\nu}_{e}$ from terrestrial reactors. Super-Kamiokande (SK) experiment first investigated another important anomaly: the atmospheric neutrino anomaly. In 1998 SK established disappearance of $\nu_{\mu}$ and $\bar{\nu}_{\mu}$ atmospheric neutrinos with more than $6 \sigma$ statistical significance which later became $\approx 17 \sigma$ in the final phase.

All these anomalies are well described by flavour oscillation a consequence of the flavour mixing, a well known phenomena in the quark sector (see appendix A). In the leptonic sector what happen is that the charged leptons fields, due to their definite masses, have no difference between the interaction fields and the actual physical fields that appear in the mass terms. For the neutrinos instead we have that interaction fields (i.e. $\nu_{e}, \nu_{\mu}, \nu_{\tau}$ ) are linear superposition of the physical ones (i.e. $\left.\nu_{1}, \nu_{2}, \nu_{3}\right)$. This difference between physical and interaction fields has another peculiar consequence for neutrinos: because of the smallness of the neutrino masses a new remarkable quantum-mechanical phenomenon can exist, that is the phenomenon of flavour oscillations. Neutrino flavour is determined by the associated lepton flavour couple to it as we showed in section (2.2). By observing neutrino oscillation it is impossible to determine the individual neutrino mass. What is actually possible to detect is a linear superposition of the 3 mass states combined by the leptonic equivalent of the CKM matrix (A-17), the Pontecorvo-MakiNakagawa-Sakata (PMNS) matrix

$$
\begin{gathered}
\left(\begin{array}{l}
\nu_{e} \\
\nu_{\mu} \\
\nu_{\tau}
\end{array}\right)=U_{P M N S}\left(\begin{array}{l}
\nu_{1} \\
\nu_{2} \\
\nu_{3}
\end{array}\right) \\
U_{P M N S} \equiv\left(\begin{array}{ccc}
c_{12} c_{13} & s_{12} c_{13} & s_{13} e^{-\mathrm{i} \delta} \\
-s_{12} c_{23}-c_{12} s_{23} s_{13} e^{\mathrm{i} \delta} & c_{12} c_{23}-s_{12} s_{23} s_{13} e^{\mathrm{i} \delta} & s_{23} c_{13} \\
s_{12} s_{23}-c_{12} c_{23} s_{13} e^{\mathrm{i} \delta} & -c_{12} s_{23}-s_{12} c_{23} s_{13} e^{\mathrm{i} \delta} & c_{23} c_{13}
\end{array}\right)
\end{gathered}
$$

with $c_{i j}=\cos \theta_{i j}, s_{i j}=\sin \theta_{i j}$ and $\delta$ being $\mathrm{CP}$ violating phase which is 
sometimes called Dirac phase.

A straightforward quantum mechanic approach can describe really well this phenomenon. We have a neutrino source producing a neutrino together with a charged lepton $l_{\alpha}$ of flavor $\alpha$. Thus, at birth, the neutrino is a $\nu_{\alpha}$. It then travels a distance $L$ to a detector. There, it interacts with a target and produces a second charged lepton $l_{\beta}$ of flavor $\beta$. Thus, at the time of its interaction in the detector, the neutrino is a $\nu_{\beta}$. If $\beta \neq \alpha$ (for example, if $l_{\alpha}=\mu$ and $l_{\beta}=\tau$ ), then, during its journey to the detector, the neutrino has morphed from $\nu_{\alpha} \rightarrow \nu_{\beta}$ as schematically illustrated in Fig.2.6.
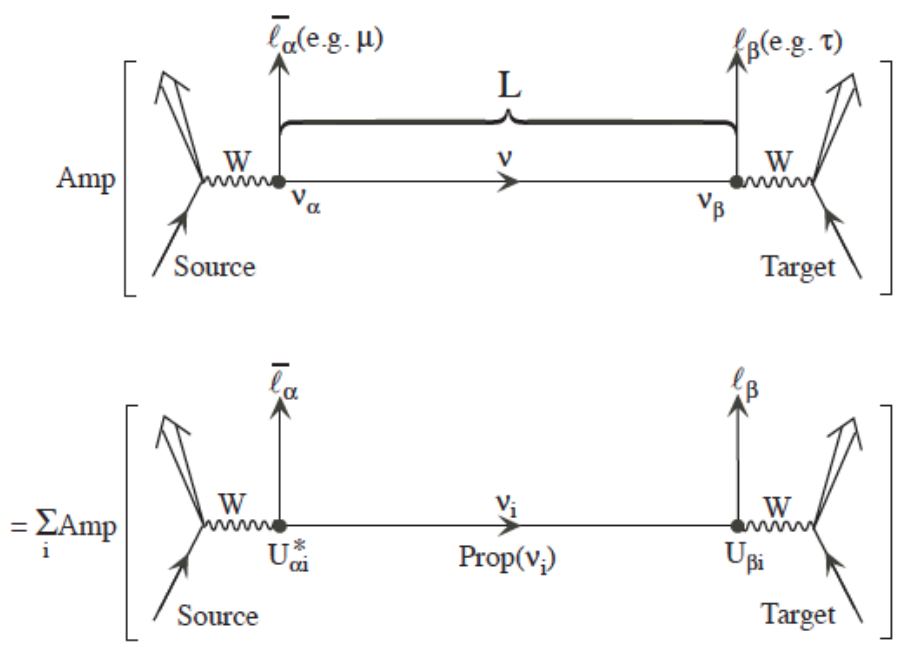

Figure 2.6: Neutrino flavour change (oscillation) in vacuum. "Amp" denotes the amplitude of the process.

The process's amplitude is a superposition of the 3 mass neutrino states contributions weighted by the PMNS elements and allow us to write (from now on we will indicate $\left.U_{P M N S} \equiv U\right)$

$$
\begin{aligned}
\left|\nu_{\alpha}\right\rangle & =\sum_{i} U_{\alpha i}\left|\nu_{i}\right\rangle \\
\operatorname{Amp}\left(\nu_{\alpha} \rightarrow \nu_{\beta}\right) & =\sum_{i=1}^{3} U_{\alpha i}^{*} \operatorname{Prop}_{l a b}\left(\nu_{i}\right) U_{\beta i},
\end{aligned}
$$

where $\operatorname{Prop}\left(\nu_{i}\right)_{l a b}$ is the neutrino propagator in the laboratory-frame (LF). The neutrino propagator between the proper time $\tau=0$ and $\tau=\tau^{\prime}$ is defined by the expression

$$
\operatorname{Prop}\left(\nu_{i}\right) \equiv\left\langle\nu_{i}(0) \mid \nu_{i}\left(\tau_{i}^{\prime}\right)\right\rangle
$$

The state $\left|\nu_{i}\left(\tau_{i}\right)\right\rangle$ is a solution of the Schrödinger equation (from now on natural units are intended $c=\hbar=1$ )

$$
\mathrm{i} \frac{\partial}{\partial \tau_{i}}\left|\nu_{i}\left(\tau_{i}\right)\right\rangle=\mathcal{H}\left|\nu_{i}\left(\tau_{i}\right)\right\rangle
$$


that in the neutrino rest-frame $(\mathrm{RF})$ it is simply

$$
\mathrm{i} \frac{\partial}{\partial \tau_{i}}\left|\nu_{i}\left(\tau_{i}\right)\right\rangle=m_{i}\left|\nu_{i}\left(\tau_{i}\right)\right\rangle
$$

and so

$$
\left|\nu_{i}\left(\tau_{i}^{\prime}\right)\right\rangle=e^{-\mathrm{i} m_{i} \tau_{i}^{\prime}}\left|\nu_{i}(0)\right\rangle \rightarrow \operatorname{Prop}\left(\nu_{i}\right)_{r e s t}=e^{-\mathrm{i} m_{i} \tau_{i}^{\prime}} .
$$

For this propagator to be useful one must re-express it in LF variables. By the Lorentz invariance one has

$$
m_{i} \tau_{i}=E_{i} t-p_{i} L
$$

Here the LF variables fixed by the experiment and equal for any mass eigenstate are $t$ and $L$ i.e. the LF time of propagation and the distance between the production and detection points. The other two LF quantities are the energy of the neutrino $E_{i}$ and its momentum $p_{i}$. Clearly either $t$ or $L$ are realistically impossible to determine so that in practice one averages over the time $t$ taken by the neutrino during its journey. Now if we take two components of a neutrino beam with energies $E_{1}$ and $E_{2}$ they will acquire a phase $\exp \left[\mathrm{i} E_{j} t\right]$ until they reach the detection point and so giving rise to an interference term $\exp \left[\mathrm{i}\left(E_{1}-E_{2}\right) t\right]$. Averaged over the unobserved travel time $t$, this factor vanishes, unless $E_{1}=E_{2}$. Thus, the only components of a neutrino beam that contribute coherently to a neutrino oscillation signal are components that have the same energy. Said that, we can rewrite the momentum $p_{i}$

$$
p_{i}=\sqrt{E^{2}-m_{i}^{2}} \approx E-\frac{m_{i}^{2}}{2 E} .
$$

This approximation is possible because for any energies considered in the experiments (from $\mathrm{MeV}$ to $\mathrm{PeV})^{2} m_{i}^{2} \ll E^{2}$. In the end the phase factor becomes

$$
\operatorname{Prop}\left(\nu_{i}\right)_{l a b}=e^{-\mathrm{i}[E(t-L)]} e^{-\mathrm{i}\left(\frac{m_{i}^{2} L}{2 E}\right)}
$$

where the first term is common to all mass component and so is irrelevant form the observation point of view.

Now using the expression of the propagator 2-56 is possible to compute the probability transition $P\left(\nu_{\alpha} \rightarrow \nu_{\beta}\right)$

$$
\begin{aligned}
P\left(\nu_{\alpha} \rightarrow \nu_{\beta}\right) & =\left|\operatorname{Amp}\left(\nu_{\alpha} \rightarrow \nu_{\beta}\right)\right|^{2} \\
& =\delta_{\alpha \beta}-4 \sum_{i>j} \Re\left(U_{\alpha i}^{*} U_{\beta i} U_{\alpha j} U_{\beta j}^{*}\right) \sin ^{2}\left(\frac{\Delta m_{i j}^{2} L}{4 E}\right) \\
& +2 \sum_{i>j} \Im\left(U_{\alpha i}^{*} U_{\beta i} U_{\alpha j} U_{\beta j}^{*}\right) \sin \left(\frac{\Delta m_{i j}^{2} L}{2 E}\right) .
\end{aligned}
$$

\footnotetext{
${ }^{2}$ There are no experimental measurements of the absolute value of the neutrino mass. The limit obtained from oscillation experiments suggest a value of $\approx 10^{-1} \mathrm{eV}[8]$
} 
For the case of $\bar{\nu}$ assuming the Charge-Parity-Time (CPT) invariance, i.e. $P\left(\nu_{\alpha} \rightarrow \nu_{\beta}\right)=P\left(\bar{\nu}_{\beta} \rightarrow \bar{\nu}_{\alpha}\right)$, one can find that the only difference is a minus sign in front of the imaginary part term.

This formula (and its manipulations) contains all neutrino phenomenology so it is important to understand the physics it contains and its mathematical properties:

- Because of the unitary evolution of the states, the oscillation probability satisfies the relations

$$
\sum_{\alpha} P\left(\nu_{\alpha} \rightarrow \nu_{\beta}\right)=1 \quad \sum_{\beta} P\left(\nu_{\alpha} \rightarrow \nu_{\beta}\right)=1 .
$$

From a physical point of view this means that neutrino oscillation does not alter the total number of neutrino produced, i.e. the total flux remain unchanged. This means that if one sum all the different flavour contributions will find the original flux intensity.

- The oscillation phase is determined by the physical quantities

$$
\Phi_{i j}=\frac{\Delta m_{i j}^{2} L}{2 E},
$$

where $\Delta m_{i j}^{2}=m_{i}^{2}-m_{j}^{2}$ are the neutrino mass squared differences and $E, L$ are quantities fixed by the experiment. This means mathematically that different combination of source neutrino energy and detector-source distance can modulate the $\sin ^{2}$ argument. Because of the averaging in the time $t \approx L$ mentioned before, the $\sin ^{2}$ is appreciable so long as its argument is of order unity or larger. For example an experiment with $L \sim 1000 \mathrm{~km}$ and $E \sim \mathrm{GeV}$ is sensitive to $\Delta m_{i j}^{2}$ down to $\sim 10^{-4} \mathrm{eV}^{2}$.

- As we said $\Phi_{i j}$ depends only on neutrino square-mass splitting but not on the individual neutrino masses. Thus, oscillation experiment can determine the neutrino squared-mass difference spectral pattern, but not how far above zero the entire pattern lies.

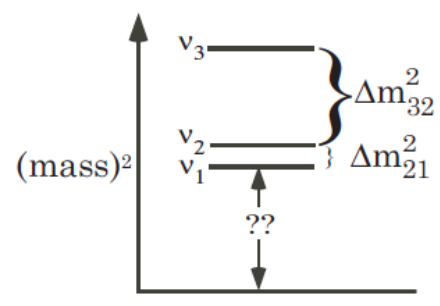

Figure 2.7: Pictorial representation of neutrino squared-mass spectral pattern. The "??" represent the unknown value of the neutrino mass scale. Figure from [9]. 
- If neutrinos were massless $\Delta m_{i j}^{2}=0$ we have $P\left(\nu_{\alpha} \rightarrow \nu_{\beta}\right)=\delta_{\alpha \beta}$ and so oscillation will not be possible. This fact stands as well for the $U_{\mathrm{s}}$ : if there were no mixing each neutrino mass state could be one-on-one associated to a lepton so that $U_{\alpha i}=0$ for $\alpha \neq i$ and again one will not observe oscillation.

An example of oscillation in vacuum is shown in Fig.2.8 where we choose to show the probability that a muon neutrino has to be detected as itself at a fixed distance $L=1100 \mathrm{~km}$ with a variable $\mathrm{GeV}$ energy.

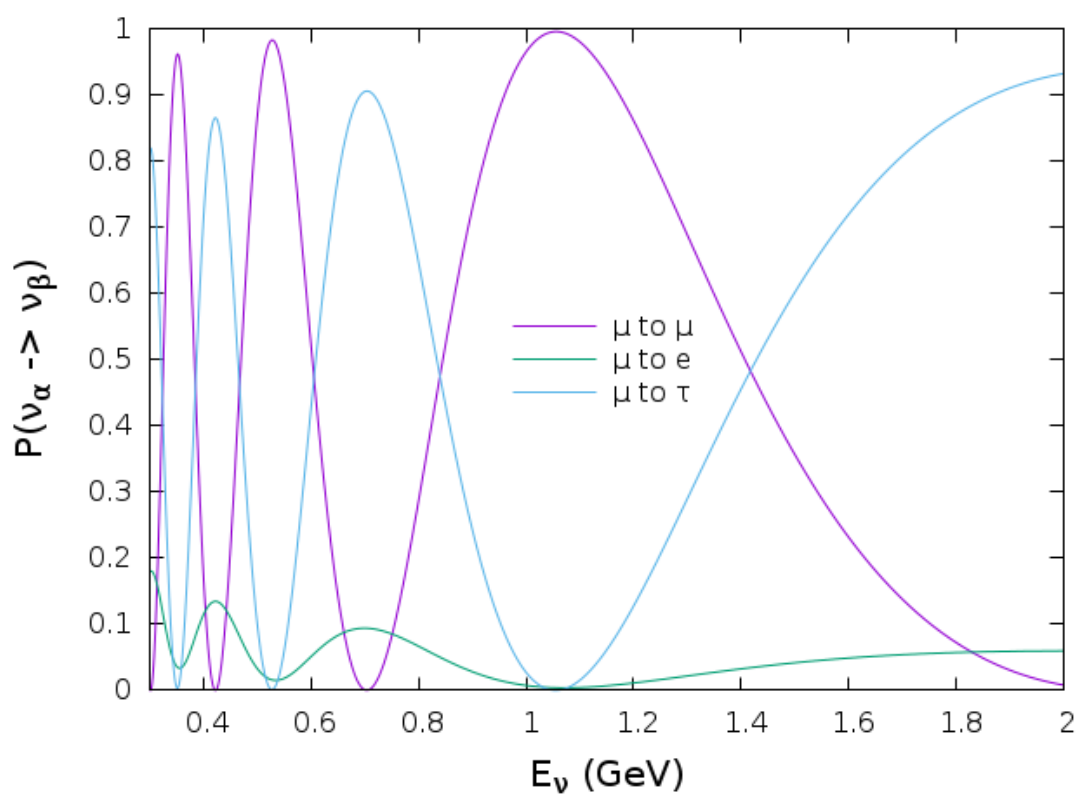

Figure 2.8: $P\left(\nu_{\mu} \rightarrow \nu_{\mu}\right), P\left(\nu_{\mu} \rightarrow \nu_{e}\right)$ and $P\left(\nu_{\mu} \rightarrow \nu_{\tau}\right)$ in vacuum at a fixed distance $L=1100 \mathrm{~km}$. 


\section{5}

\section{Neutrino Oscillation in Matter}

When active flavour neutrinos propagate in matter their evolution equation is affected by effective potentials due to the coherent interactions with the medium through coherent forward elastic weak $C C$ and $N C$ scatterings [10]. In Fig.2.9 we show the tree level Feynman diagrams of $C C$ and $N C$ scattering.
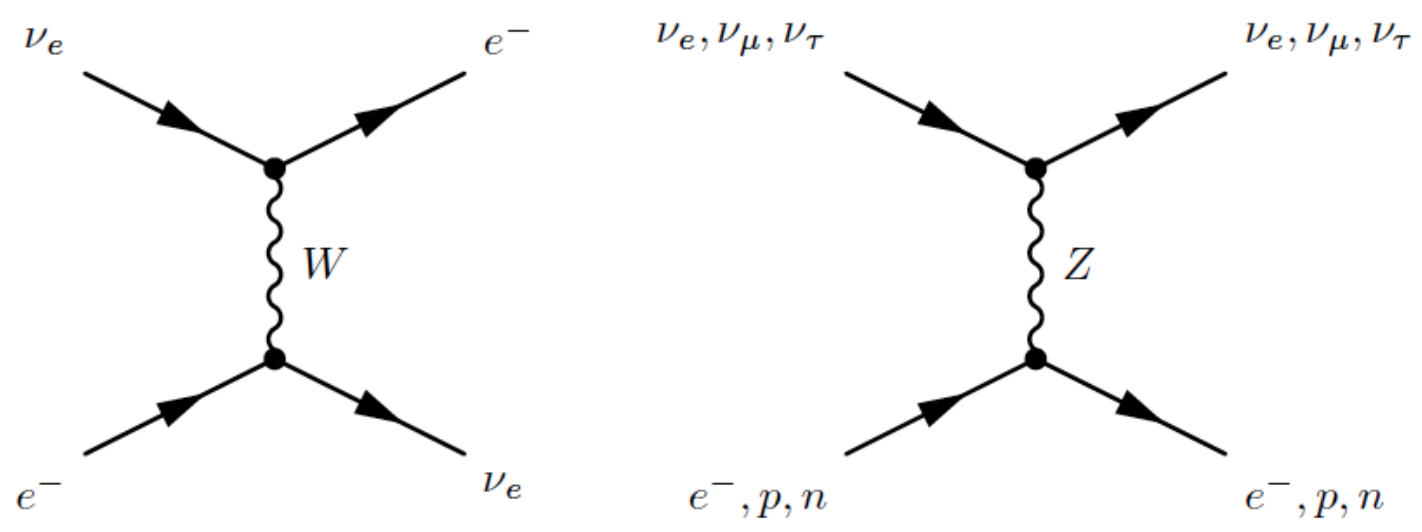

Figure 2.9: Feynman diagrams of the coherent forward elastic scattering processes that generate the $C C$ potential $V_{C C}$ through $\mathrm{W}$ exchange and the $N C$ potential $V_{N C}$ through $Z$ exchange. Figure taken from [2].

These interactions act on the neutrino propagation like a refraction index for the light in a medium. Calculating the cross section and averaging over the matter distribution of the medium, the $C C$ and $N C$ interaction give rise to respectively the effective potential $V_{C C}$ and $V_{N C}$

$$
V_{C C}=\sqrt{2} G_{F} N_{e} \quad V_{N C}=-\frac{1}{2} \sqrt{2} G_{F} N_{f},
$$

where $G_{F}$ is the Fermi constant, $N_{e}$ is the electron number density of the medium and $N_{f}$ is the fermion number density of the medium. In astrophysical environments with low temperature and density, matter is composed of neutrons, protons, and electrons. Since electrical neutrality implies an equal number density of protons and electrons, the neutral-current potentials of protons and electrons cancel each other so that we can substitute $N_{f}$ with simply $N_{n}$ with this being the neutron density. With this in mind we can rewrite the Schrödinger equation that describe the neutrino propagation as

$$
\mathrm{i} \frac{\partial}{\partial t}\left|\nu_{\alpha}\right\rangle=\mathcal{H}_{m}\left|\nu_{\alpha}\right\rangle
$$

where $\mathcal{H}_{m}=\mathcal{H}_{0}+\mathcal{H}_{I}$ with $\mathcal{H}_{0}$ being diagonalized by the vacuum mass states $\left|\nu_{i}\right\rangle$

$$
\mathcal{H}_{0}\left|\nu_{i}\right\rangle=E_{i}\left|\nu_{i}\right\rangle
$$


and $\mathcal{H}_{I}$ being the interaction Hamiltonian defined by

$$
\mathcal{H}_{I}=\sqrt{2} G_{F}\left(N_{e} \delta_{\alpha e}+\mathbb{1} N_{n} \frac{1}{2}\right) .
$$

As we have seen in the previous section, all the neutrinos that we are physically interested can be considered ultrarelativistic because they carry energy of order of at least tens of $\mathrm{MeV}$. In this range we can write

$$
E_{i} \simeq E+\frac{m_{i}^{2}}{2 E} \quad t \simeq x
$$

with $x$ being the neutrino position in a matter slab of linear dimension $L$ taking values in the interval $[0, L]$. Putting all together in matrix form we have

$$
\mathrm{i} \frac{\mathrm{d}}{\mathrm{d} x}\left(\begin{array}{c}
\nu_{e} \\
\nu_{\mu} \\
\nu_{\tau}
\end{array}\right)=\frac{1}{2 E}\left[U\left(\begin{array}{ccc}
0 & 0 & 0 \\
0 & \Delta m_{21}^{2} & 0 \\
0 & 0 & \Delta m_{31}^{2}
\end{array}\right) U^{\dagger}+\left(\begin{array}{ccc}
2 \sqrt{2} E G_{F} N_{e}(x) & 0 & 0 \\
0 & 0 & 0 \\
0 & 0 & 0
\end{array}\right)\right]\left(\begin{array}{c}
\nu_{e} \\
\nu_{\mu} \\
\nu_{\tau}
\end{array}\right),
$$

where we have used the property of the evolution equation to subtract the diagonal term $\mathbb{1} V_{N C}$ without changing the final result. The three generation case with a generic density function $N_{e}(x)$ has no analytical solution and can be resolved just by a numerical approach. For constant density approximation there is a closed solution first obtained by Zaglauer and Schwarzer [11]. In the next subsection we will show the effect of the presence of the matter potential for the simplified case of two generation. 


\subsection{1}

\section{Two Generation Case: The MSW Effect}

In the case of just two neutrinos their flavour states can be expressed in terms of the mass states in the form

$$
\left(\begin{array}{c}
\nu_{e} \\
\nu_{\mu}
\end{array}\right)=U\left(\begin{array}{l}
\nu_{1} \\
\nu_{2}
\end{array}\right)
$$

where

$$
U=\left(\begin{array}{cc}
c_{12} & s_{12} \\
-s_{12} & c_{12}
\end{array}\right)
$$

In this case eq.(2-65) reduces to

$$
\mathrm{i} \frac{\mathrm{d}}{\mathrm{d} x}\left(\begin{array}{l}
\nu_{e} \\
\nu_{\mu}
\end{array}\right)=\frac{1}{2 E}\left[U\left(\begin{array}{cc}
0 & 0 \\
0 & \Delta m_{21}^{2}
\end{array}\right) U^{\dagger}+\left(\begin{array}{cc}
2 \sqrt{2} E G_{F} N_{e}(x) & 0 \\
0 & 0
\end{array}\right)\right]\left(\begin{array}{l}
\nu_{e} \\
\nu_{\mu}
\end{array}\right) .
$$

The total Hamiltonian can be written as

$$
\mathcal{H}_{m}=\frac{1}{4 E}\left(\begin{array}{cc}
-\Delta m_{21}^{2} \cos 2 \theta_{12}+A_{C C} & \Delta m_{21}^{2} \sin 2 \theta_{12} \\
\Delta m_{21}^{2} \sin 2 \theta_{12} & \Delta m_{21}^{2} \cos 2 \theta_{12}-A_{C C}
\end{array}\right)
$$

where $A_{C C}=2 \sqrt{2} E G_{F} N_{e}(x)$. This matrix can be diagonalized by changing the flavour states to a matter state as

$$
\left(\begin{array}{c}
\nu_{e} \\
\nu_{\mu}
\end{array}\right)=U_{m}\left(\begin{array}{c}
\nu_{1}^{m} \\
\nu_{2}^{m}
\end{array}\right) \quad U_{m}^{\dagger} \mathcal{H}_{m} U_{m}=\mathcal{H}_{m}^{\text {diag }}
$$

where

$$
\mathcal{H}_{m}^{\text {diag }}=\frac{1}{4 E} \operatorname{diag}\left(-\Delta m_{21 m}^{2}, \Delta m_{21 m}^{2}\right) .
$$

The matrix $U_{m}$ has the same structure as $U$ but now the effective angle in matter $\theta_{12}^{m}$ is related to the vacuum $\theta_{12}$ by the relations

$$
\cos 2 \theta_{12}^{m}=\frac{\Delta m_{21}^{2} \cos 2 \theta_{12}-A_{C C}}{\Delta m_{21 m}^{2}} \quad \sin 2 \theta_{12}^{m}=\frac{\Delta m_{21}^{2} \sin 2 \theta_{12}}{\Delta m_{21 m}^{2}},
$$

where $\Delta m_{21 m}^{2}$ is the effective squared mass difference in matter

$$
\Delta m_{21 m}^{2}=\sqrt{\left(\Delta m_{21}^{2} \cos 2 \theta_{12}-A_{C C}\right)^{2}+\left(\Delta m_{21}^{2} \sin 2 \theta_{12}\right)^{2}} .
$$

In 1985 Mikheyev and Smirnov [12] discovered a new phenomenon which is a resonance when $A_{C C}$ becomes equal to

$$
A_{C C}^{R}=\Delta m_{21}^{2} \cos 2 \theta_{12}
$$

At the resonance the effective mixing angle is equal to $\pi / 4$, i.e. the mixing is maximal, leading to the possibility of total transitions between the two flavours if the adiabaticity condition is satisfied. This mechanism is called the MSW effect (Mikheyev, Smirnov, and Wolfenstein) and plays a crucial role in the explanation of the solar neutrino problem (see next chapter). 
We can then rewrite eq.(2-68) in matter mass eigenstate basis to have

$$
\mathrm{i} \frac{\mathrm{d}}{\mathrm{d} x}\left(\begin{array}{c}
\nu_{1}^{m} \\
\nu_{2}^{m}
\end{array}\right)=\frac{1}{4 E}\left(\begin{array}{cc}
-\Delta m_{21 m}^{2} & -4 E \mathrm{id} \theta_{12}^{m} / \mathrm{d} x \\
4 E \mathrm{id} \theta_{12}^{m} / \mathrm{d} x & \Delta m_{21 m}^{2}
\end{array}\right)\left(\begin{array}{l}
\nu_{1}^{m} \\
\nu_{2}^{m}
\end{array}\right) .
$$

The off diagonal terms are responsible for the transition $\nu_{1}^{m} \rightarrow \nu_{2}^{m}$ during the evolution in matter. In the case, for example, that the constant density distribution term is null then the evolution of the amplitudes of the effective massive neutrinos simply become

$$
\begin{aligned}
& \left|\nu_{1}^{m}(x)\right\rangle=\exp \left(\mathrm{i} \frac{\Delta m_{21 m}^{2}}{4 E}\right)\left|\nu_{1}^{m}(0)\right\rangle \\
& \left|\nu_{2}^{m}(x)\right\rangle=\exp \left(-\mathrm{i} \frac{\Delta m_{21 m}^{2}}{4 E}\right)\left|\nu_{2}^{m}(0)\right\rangle .
\end{aligned}
$$

Now we have all the elements to calculate the transition probability from one flavour to another in matter. Let us take for example the case of a transition $\nu_{e} \rightarrow \nu_{\mu}$ from a point with a constant electron density to a point that is in vacuum, so we have

$P\left(\nu_{e} \rightarrow \nu_{\mu}\right)(x)=\left|\left\langle\nu_{\mu} \mid \nu_{e}(x)\right\rangle\right|^{2}=\left|\left(-\left\langle\left.\nu_{1}\left|s_{12}+\left\langle\nu_{2}\right| c_{12}\right)\left(c_{12}^{m} \phi\left|\nu_{1}^{m}(0)\right\rangle+s_{12}^{m} \phi^{*}\left|\nu_{2}^{m}(0)\right\rangle\right)\right|^{2}\right.\right.\right.$,

where $\phi=\exp \left(\mathrm{i} \frac{\Delta m_{21 m}^{2}}{4 E}\right)$. To conclude this calculation we just need to find the relation between the states $\nu_{i}$ and $\nu_{i}^{m}$. Those are simply deducible if we consider the condition that in vacuum $\nu_{i}^{m} \equiv \nu_{i}$ so that

$$
\left(\begin{array}{c}
\nu_{1}^{m} \\
\nu_{2}^{m}
\end{array}\right)=\left(\begin{array}{cc}
c_{12} c_{12}^{m}+s_{12} s_{12}^{m} & c_{12}^{m} s_{12}-s_{12}^{m} c_{12} \\
-\left(c_{12}^{m} s_{12}-s_{12}^{m} c_{12}\right) & c_{12} c_{12}^{m}+s_{12} s_{12}^{m}
\end{array}\right)\left(\begin{array}{c}
\nu_{1} \\
\nu_{2}
\end{array}\right) .
$$

After a straightforward calculation we obtain

$$
P\left(\nu_{e} \rightarrow \nu_{\mu}\right)(x)=\sin ^{2} 2 \theta_{12}^{m} \sin ^{2}\left(\frac{\Delta m_{21 m}^{2} x}{4 E}\right),
$$

that mimic exactly the functional form of the two flavour vacuum case where we are replacing the vacuum $\theta_{12}$ with the effective one in matter. In the case where $N_{e}(x)$ is not constant one can still find an analytical solution if the offdiagonal terms are negligible with respect to the diagonal part. To quantify this effect is useful to introduce what is called adiabaticity parameter

$$
\gamma=\frac{\Delta m_{21 m}^{2}}{\left|4 E \mathrm{~d} \theta_{12}^{m} / \mathrm{d} x\right|}
$$

that is the ratio between the diagonal term the off-diagonal one. If $\gamma \gg 1$ in all the points of the neutrino trajectory then the evolution is called adiabatic and the two matter eigenstates evolve independently. This condition can be 
also formulated in terms of oscillation length. If we define

$$
L_{m}^{o s c}=\frac{4 E \pi}{\Delta m_{21 m}^{2}}
$$

we have that the condition $\gamma \gg 1$ becomes

$$
d \equiv\left|\pi \mathrm{d} \theta_{12}^{m} / \mathrm{d} x\right| \gg L_{m}
$$

This means that the region where we have a density variation must be several times bigger than the oscillation length in matter so that this effect can be averaged out.

The adiabaticity condition allow us to write the eigenstates evolution in the form

$$
\begin{aligned}
& \left|\nu_{1}^{m}(x)\right\rangle=\exp \left(\mathrm{i} \int_{0}^{x} \frac{\Delta m_{21 m}^{2}}{4 E} \mathrm{~d} x^{\prime}\right)\left|\nu_{1}^{m}(0)\right\rangle \\
& \left|\nu_{2}^{m}(x)\right\rangle=\exp \left(-\mathrm{i} \int_{0}^{x} \frac{\Delta m_{21 m}^{2}}{4 E} \mathrm{~d} x^{\prime}\right)\left|\nu_{2}^{m}(0)\right\rangle .
\end{aligned}
$$

In this case we can still calculate an exact form for the flavour transition, for example $\nu_{e} \rightarrow \nu_{e}$ from a point with matter to a point in vacuum $P\left(\nu_{e} \rightarrow \nu_{e}\right)(x)=\left|\left\langle\nu_{e} \mid \nu_{e}(x)\right\rangle\right|^{2}=\left|\left(\left\langle\left.\nu_{1}\left|c_{12}+\left\langle\nu_{2}\right| s_{12}\right)\left(c_{12}^{m} \phi\left|\nu_{1}^{m}(0)\right\rangle+s_{12}^{m} \phi^{*}\left|\nu_{2}^{m}(0)\right\rangle\right)\right|^{2}\right.\right.\right.$, (2-84) where now $\phi=\exp \left(\mathrm{i} \int_{0}^{x} \frac{\Delta m_{21 m}^{2}}{4 E} \mathrm{~d} x^{\prime}\right)$. Performing the same calculation as before we obtain

$$
\begin{aligned}
P\left(\nu_{e} \rightarrow \nu_{e}\right)(x) & =\frac{1}{2}+\frac{1}{2} \cos 2 \theta_{12}^{m} \cos 2 \theta_{12} \\
& +\frac{1}{2} \sin 2 \theta_{12}^{m} \sin 2 \theta_{12} \cos \left(\int_{0}^{x} \frac{\Delta m_{21 m}^{2}}{4 E} \mathrm{~d} x^{\prime}\right)
\end{aligned}
$$

This formula can be simplified if we think that the distance between the source and a detector is huge and the cos term can be averaged out, so we have

$$
\bar{P}\left(\nu_{e} \rightarrow \nu_{e}\right)=\frac{1}{2}+\frac{1}{2} \cos 2 \theta_{12}^{m} \cos 2 \theta_{12} .
$$




\section{6}

\section{Leptonic CP Violation in Neutrino Oscillation Framework}

In particle physics CPT conservation is assumed to stand for any type of phenomenon, and as we mentioned in the previous section, the probability formula described in eq.(2-57) is unchanged after a CPT transformation.

But what is the effect of applying a $\mathrm{CP}$ or $\mathrm{T}$ or CPT transformation to a neutrino? First of all a CP transformation relate neutrinos with antineutrinos

$$
\nu_{\alpha} \stackrel{C P}{\longleftrightarrow} \bar{\nu}_{\alpha}
$$

and so the transition probability change as

$$
\nu_{\alpha} \rightarrow \nu_{\beta} \stackrel{C P}{\longrightarrow} \bar{\nu}_{\alpha} \rightarrow \bar{\nu}_{\beta}
$$

In the case of a $\mathrm{T}$ transformation what happen is that the rule of $\alpha$ and $\beta$ are interchanged in neutrino and in the antineutrino case

$$
\nu_{\alpha} \rightarrow \nu_{\beta} \stackrel{T}{\longleftrightarrow} \nu_{\beta} \rightarrow \nu_{\alpha} \quad \bar{\nu}_{\beta} \rightarrow \bar{\nu}_{\alpha} \stackrel{T}{\longleftrightarrow} \bar{\nu}_{\alpha} \rightarrow \bar{\nu}_{\beta} .
$$

From the probability formula point of view this two type of transformation has the same effect, i.e. $U_{\alpha i} \rightarrow U_{\alpha i}^{*}$, so that a CPT transformation

$$
\nu_{\alpha} \rightarrow \nu_{\beta} \stackrel{C P T}{\longleftrightarrow} \bar{\nu}_{\beta} \rightarrow \bar{\nu}_{\alpha} .
$$

A full scheme of all the possible combination is shown in Fig.2.10.

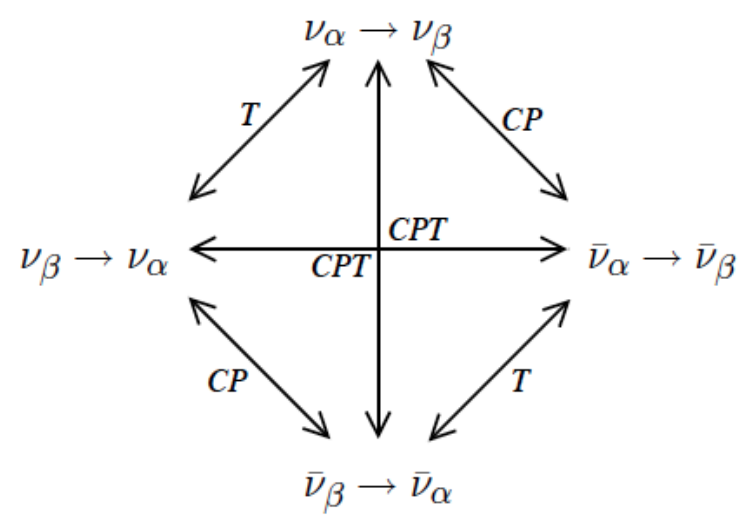

Figure 2.10: Scheme of the $\mathrm{CPT}, \mathrm{CP}$, and $\mathrm{T}$ transformations that relate different flavour transition channels.

So CPT is a symmetry for the transition probability, that is not true for $\mathrm{CP}$ or $\mathrm{T}$ transformation only. If we define the CP asymmetry

$$
A_{\alpha \beta}^{C P}=P\left(\nu_{\alpha} \rightarrow \nu_{\beta}\right)-P\left(\bar{\nu}_{\alpha} \rightarrow \bar{\nu}_{\beta}\right)
$$

and inserting the explicit expression (2-57) we have 


$$
A_{\alpha \beta}^{C P}=4 \sum_{i>j} \Im\left(U_{\alpha i}^{*} U_{\beta i} U_{\alpha j} U_{\beta j}^{*}\right) \sin \left(\frac{\Delta m_{i j}^{2} L}{2 E}\right) .
$$

From the unitarity of $U$ and using the relations

$$
\sum_{j} U_{\alpha j} U_{\beta j}^{*}=\delta_{\alpha \beta}, \quad \sum_{\alpha} U_{\alpha j} U_{\alpha k}^{*}=\delta_{j k},
$$

it is possible to find some interesting properties of the sin coefficients in (2-92). Let us define

$$
J_{i j}^{\alpha \beta}=-\Im\left(U_{\alpha i}^{*} U_{\beta i} U_{\alpha j} U_{\beta j}^{*}\right)
$$

these are the famous Jarlskog coefficients, and due to the unitarity conditions they are not all independent

$$
\begin{gathered}
J_{12}^{\alpha \beta}=J_{23}^{\alpha \beta}=J_{31}^{\alpha \beta}, \\
J_{i j}^{e \mu}=J_{i j}^{\mu \tau}=J_{i j}^{\tau e}, \\
J_{i j}^{\alpha \beta}=-J_{j i}^{\alpha \beta}=-J_{i j}^{\beta \alpha}, \\
J_{i i}^{\alpha \beta}=-J_{i j}^{\alpha \alpha}=0 .
\end{gathered}
$$

In the case of three generation it turns out that there is just one independent coefficient the Jarlskog parameter[13]. Its explicit form in the parametrization shown in $(2-47)$ is

$$
J_{12}^{e \mu}=J=c_{13}^{2} s_{13} s_{12} c_{12} s_{23} c_{23} \sin \delta .
$$

It means that the asymmetry term can be rearranged in a single term

$$
A_{\alpha \beta}^{C P}=2 J\left[\sin \left(\frac{\Delta m_{21}^{2} L}{2 E}\right)+\sin \left(\frac{\Delta m_{32}^{2} L}{2 E}\right)-\sin \left(\frac{\Delta m_{31}^{2} L}{2 E}\right)\right] .
$$

The sines in parenthesis can be rewritten as

$$
\begin{aligned}
& \sin a+\sin b-\sin (a+b)=2 \sin \left(\frac{a+b}{2}\right) \cos \left(\frac{a-b}{2}\right)-\sin \left(2 \frac{a+b}{2}\right)= \\
= & 2 \sin \left(\frac{a+b}{2}\right)\left(\cos \left(\frac{a+b}{2}\right)-\cos \left(\frac{a-b}{2}\right)\right)=4 \sin \left(\frac{a+b}{2}\right) \sin \left(\frac{a}{2}\right) \sin \left(\frac{b}{2}\right),
\end{aligned}
$$

and so the final expression is

$$
A_{\alpha \beta}^{C P}=\left(1-\delta_{\alpha \beta}\right) 16 J \sin \left(\frac{\Delta m_{31}^{2} L}{2 E}\right) \sin \left(\frac{\Delta m_{32}^{2} L}{2 E}\right) \sin \left(\frac{\Delta m_{21}^{2} L}{2 E}\right) .
$$

This quantity summarizes all the condition that allow $\mathrm{CP}$ violation to be observed

- It clearly shows that CP violation is not observable in survival oscillation 
transition, i.e. $\alpha=\beta$.

- All the mixing angles must be different from zero.

- The value of $\delta$ must be different from zero or $\pi$.

- One has to realize an experiment where all the three phases $\Delta m_{i j}^{2} L / 2 E$ are appreciably different from zero. 


\section{3}

\section{Current Status of Neutrino Physics}

As we briefly discussed in the previous chapter, various experiments established that neutrino flavour is not conserved during their propagation. Almost all the results are well described by the $3-\nu$ model, i.e. the existence of a leptonic mixture of three active type of neutrinos parametrized as we have shown in (2-47). The derived flavour oscillation probability function (2-57) is determined by 6 parameters: the 3 mixing angles $\theta_{12}, \theta_{13}, \theta_{23}$, the 2 mass splitting $\Delta m_{21}^{2}, \Delta m_{31}^{2} / \Delta m_{32}^{2}$ and the CP violation phase $\delta$ for the case we are considering Dirac neutrinos. Even if flavour oscillation phenomenon are driven by the splitting of the neutrino mass eigenstates, in principle, it is possible to determine also the ordering of the mass eigenstates. The current available data do not allow do determine with high confidence level the mass ordering so this is still a open question leaving to two possible scenarios : one called Normal Ordering (NO) where $m_{1}<m_{2}<m_{3}$ and the other one is called Inverted Ordering (IO) with $m_{3}<m_{1}<m_{2}$. The choice of $m_{1}<m_{2}$ is not casual and it is motivated by solar neutrino experiment results (see later in the chapter). In order to measure all these parameters, very different experimental set up are required due to the very different physical processes involved in the production and detection of the different neutrino types. One can separate the study of all these different combinations in three big families: solar neutrinos, atmospheric and accelerator neutrinos and reactor neutrino. This separation is also motivated by the fact that these groups of experiments are mainly sensitive to the 1-2, 2-3 and 1-3 neutrino sectors respectively ${ }^{1}$.

In this chapter we will sum up the most important physical characteristics of each family and present an updated global analysis of all the last experimental results.

\section{1}

\section{Solar Neutrinos}

Solar neutrino studies have driven the interest in neutrino physics in the last 50 years. The first experimental indication that neutrino physics was

${ }^{1}$ This separation is not so strict because for example KamLAND (despite reactor experiments) is mainly sensitive to 1-2 sector and accelerator experiments is also sensitive to the $1-3$ sector 
deeper than the SM predictions was observed by the Homestake experiment [4] in 1968. The deficit of solar $\nu_{e}$ flux with respect to the one predicted by Bahcall [5], with the Pontecorvo [14] inferences on the possibility that neutrinos could oscillate, gave the first hint that neutrinos are massive particles.

Inside the Sun neutrinos are produced by nuclear reactions in the ppchain and CNO cycle. The main reactions and the flux as a function of neutrino energy are shown in Fig.3.1.

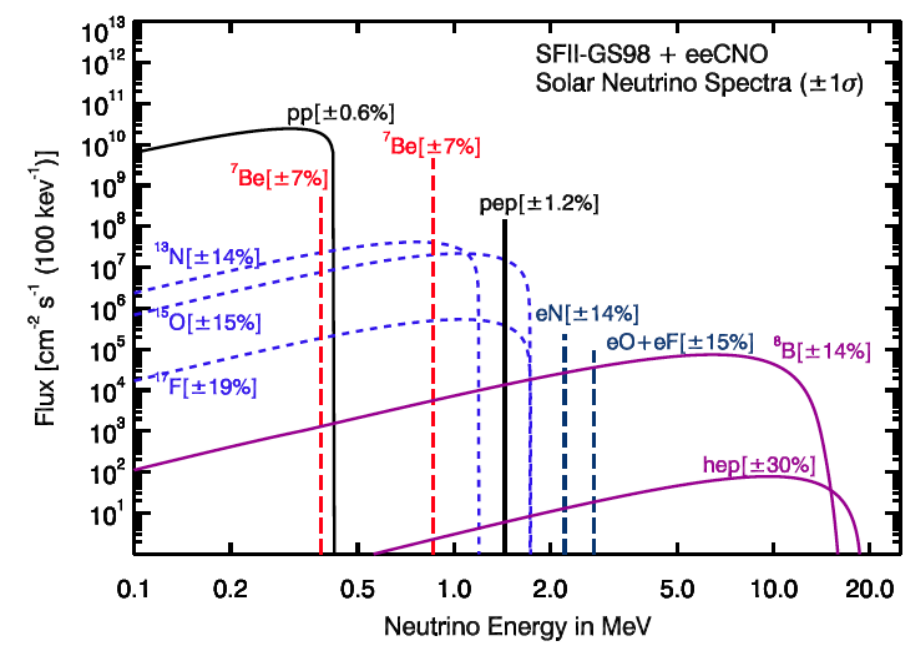

Figure 3.1: The neutrino fluxes are given in units of $\mathrm{cm}^{-2} \mathrm{~s}^{-1} \mathrm{MeV}^{-1}$ for continuum spectra and $\mathrm{cm}^{-2} \mathrm{~s}^{-1} \mathrm{MeV}^{-1}$ for line spectra. This figure is taken from [8].

The most investigated channel in solar neutrino physics has been the ${ }^{8} B$. The reason is quite simply explained looking back to Fig.3.1 where one can see that there is a region between $\sim 3 \mathrm{MeV}$ and $\sim 11 \mathrm{MeV}$ where the neutrino flux has the best signal to noise ratio, therefore, it is the simplest channel to look experimentally. The nuclear reaction in the ${ }^{8} B$ channel is

$$
{ }^{8} B \rightarrow{ }^{8} \mathrm{Be}^{*}+e^{+}+\nu_{e}
$$

so that when neutrinos are produced as $100 \%$ electron flavour and the reaction happen almost at the center of the Sun. Because the energy of Solar neutrinos are of the order of few $\mathrm{MeV}$ one will be able to detect just electron neutrinos and the oscillation probability channel that we can investigate in this case is $P_{e e}=P\left(\nu_{e} \rightarrow \nu_{e}\right)$. The propagation of $\nu_{e}$ from the Sun to the Earth can be separated in three steps:

1) Neutrino state produced as $\nu_{e}$ in the central regions of the Sun propagates as the system of eigenstates of the Hamiltonian, $\nu_{i}^{m}$. Admixtures of the eigenstates are determined by the mixing in matter in the production region. The eigenstates propagate independently of each other and 
transform into corresponding mass eigenstates when arriving at the surface of the Sun: $\nu_{i}^{m} \rightarrow \nu_{i}$.

2) The mass eigenstates propagate without changes to the surface of the Earth. The coherence between these states is lost and oscillations are irrelevant.

3) Once at Earth the mass states $\nu_{i}$ split (decomposed) into the eigenstates in matter of the Earth and oscillate propagating inside the Earth to the detector.

For what it concern the first part of the propagation we already discussed the case of neutrinos in matter in Chp.1. One can solve the problem analytically when electron density profile is constant or varies adiabatically. From the solar model studies we know that the $N_{e}(x)$ profile is of the form

$$
N_{e}(x)=N_{e}\left(x_{0}\right) \exp \left\{-\frac{x-x_{0}}{r_{0}}\right\}
$$

where $x-x_{0}=d$ is the distance traveled by the neutrino in the Sun, $N_{e}\left(x_{0}\right)$ is the electron number density at the point of $\nu_{e}$ production in the Sun, i.e. $\sim 100 N_{A} \mathrm{~cm}^{-3}$, and $r_{0} \sim 0.1 R_{\odot}$ is the scale-height of the change of $N_{e}(x)$ [8]. This means that the interior region of the Sun where neutrinos propagates follow the adiabaticity condition (2-80). In this case we have no transition $\nu_{1}^{m} \leftrightarrow \nu_{2}^{m}$ and each eigenstate evolve independently. The full calculation of the transition probability is shown in Chp.1 in the simplified case of two flavour. Here we present the final result that is

$$
P_{e e}^{\odot}=P\left(\nu_{e} \rightarrow \nu_{e}\right)=\frac{1}{2}+\frac{1}{2} \cos 2 \theta_{12}^{m} \cos 2 \theta_{12},
$$

where the symbol $\odot$ stands for "inside the Sun". As we have seen before, what arrive to the surface of the Sun are decoherent $\nu_{i}$ eigenstates that will travel to the Earth without oscillating. It is not difficult to estimate what is the preferential $\nu_{i}$ eigenstate in which $\nu_{e}$ will come out from the Sun. The electron density in the production region is approximately $\sim 100 N_{A} \mathrm{~cm}^{-} 3$ and we can write what is the mixture of $\nu_{i}$ in $\nu_{e}$

$$
\left(\begin{array}{l}
\nu_{1}^{m} \\
\nu_{2}^{m}
\end{array}\right)=\left(\begin{array}{cc}
c_{12}^{m} & -s_{12}^{m} \\
s_{12}^{m} & c_{12}^{m}
\end{array}\right)\left(\begin{array}{l}
\nu_{e} \\
\nu_{\mu}
\end{array}\right) \approx\left(\begin{array}{cc}
0 & -1 \\
1 & 0
\end{array}\right)\left(\begin{array}{l}
1 \\
0
\end{array}\right)=\left(\begin{array}{l}
0 \\
1
\end{array}\right),
$$

where we used the fact that at high density $\theta_{12}^{m} \sim \pi / 2$.

So neutrinos merge from the Sun in their heaviest mass eigenstate until they reach the Earth ${ }^{2}$. Here $\nu_{2}$ is decomposed in terms of Earth mass eigenstates,

${ }^{2}$ The study of the neutrino propagation in Earth is a really deep topic and a proper study is beyond the focus of this section. We will just recall the main features that are functional to our discussion. 
giving rise to what is called regeneration. For the neutrinos at ${ }^{8} B$ channel the effect is observable only in the "night", i.e. when neutrinos cross the Earth, because their oscillation length is of the order of $300 \mathrm{~km}$ [15]. With this in mind the survival probability of a $\nu_{e}$ produced inside the Sun to be detected at the Earth as itself can be written as [8]

$$
P_{e e}^{S E}=P_{e e}^{\odot}+\left(1-2 P_{e e}^{\odot}\right) \frac{P_{e 2}-\sin ^{2} \theta_{12}}{\cos 2 \theta_{12}},
$$

where $P_{e 2}=\left|\left\langle\nu_{e} \mid \nu_{2}\right\rangle\right|^{2}$.

From the study of the distortion of $P_{e e}^{S E}$ it is possible to determine $\Delta m_{21}^{2}$ and the mixing angle $\theta_{12}$. In Fig.3.2 we show $P_{e e}$ against the experimental data of the principal experiments [15]. Different values of $\Delta m_{21}^{2}$ shift the curve otherwise different values of $\theta_{12}$ change the shape. From the experimental measurement of $P_{e e}^{S E}$ it is possible to fix order $m_{1}<m_{2}$. The sign of the matter effect in Earth propagation and the portion of $e$ flavour in the heaviest eigenstate fix $\Delta m_{21}^{2}>0[9,15]$.

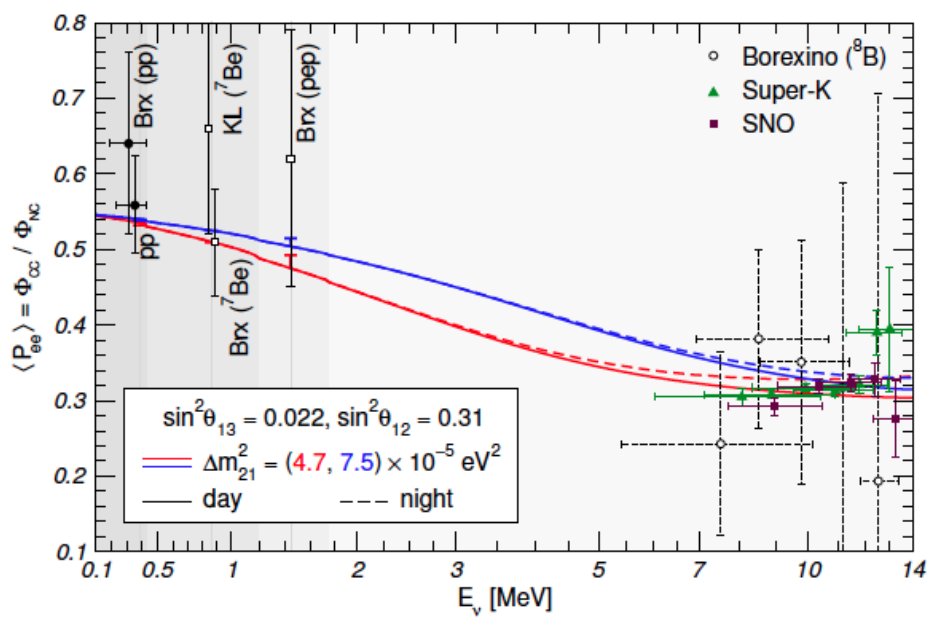

Figure 3.2: Dependence of the probability $P_{e e}$ integrated over the day and the night time periods. This figure is taken from [15].

In Fig.3.3 we show the global fit of the most important Solar neutrino experiments and a comparison between this fit and the KamLAND result. The KamLAND experiment is a long baseline reactor neutrino one studying the $P\left(\bar{\nu}_{e} \rightarrow \bar{\nu}_{e}\right)$ survival channel. They have perfect agreement with respect the $\theta_{12}$ values but a $2 \sigma$ discrepancy when it comes to $\Delta m_{21}^{2}$.

The origin of this difference is one of the open questions in neutrino physics that can be investigated by next generation Solar neutrino experiments. The sub \% level of precision that SNO+JUNO and HK experiments [16-18] will test the $3 \nu$ model as well as physics beyond the standard model like Sterile 


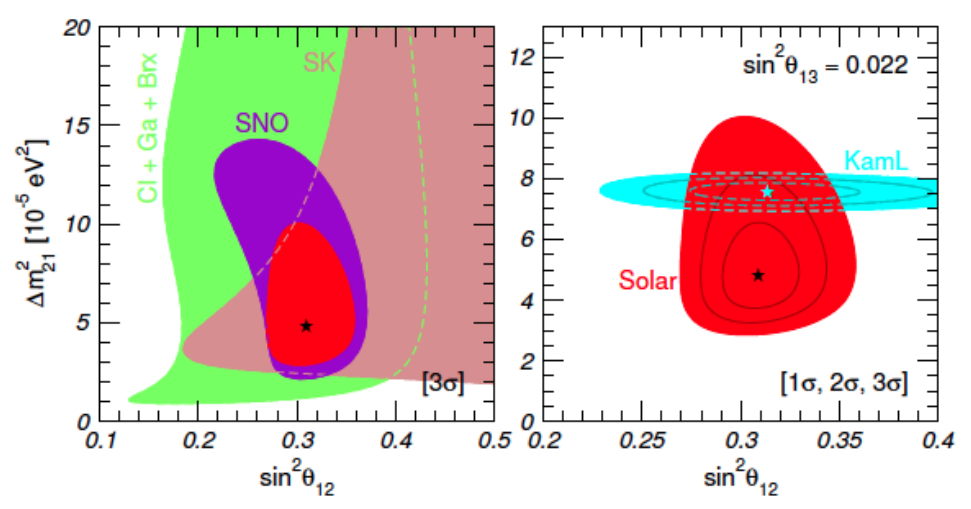

Figure 3.3: The right panel shows the allowed regions of $\theta_{12}$ and $\Delta m_{21}^{2}$ from the global fit of the solar neutrino data (red) as well as KamLAND (blue), for $\theta_{13}$ fixed to the best fit of the reactor experiments. In the left panel are shown also regions restricted by individual experiments (Chlorine, Gallex, Borexino). This figure is taken from [15].

neutrinos, Non Standard Interaction (NSI) or even the possibility to put more stringent limit on neutrino decay lifetime.

\section{2}

\section{Atmospheric and Accelerator Neutrinos}

Atmospheric neutrinos are produced by the decays of $\pi$ (pions) and $K$ (kaons) mesons produced in the nuclear interactions of the primary component of cosmic rays in the atmosphere. The principal reaction with the respective branching ratios are showed in Table 3.1 that we took from [8].

From an analysis of these reactions one can already have a rough idea of what should be the flux ratio between $\nu_{\mu}, \bar{\nu}_{\mu}, \nu_{e}$ and $\bar{\nu}_{e}$ at the Earth surface. For energies $<1 \mathrm{GeV}$ the ratio $\left(\nu_{\mu}+\bar{\nu}_{\mu}\right) /\left(\nu_{e}+\bar{\nu}_{e}\right) \approx 2$ because all the produced muons will decay. So for higher energies this ratio will increase because an increasing fraction of muons do not decay before reaching the ground and being absorbed. We also have an expected equal flux ratios of $\nu_{\mu} / \bar{\nu}_{\mu}$ and $\nu_{e} / \bar{\nu}_{e}$ that should reflect the $\pi^{+} / \pi^{-}$symmetry, even in reality we should have a little excess of $\pi^{+}$because the dominance of protons in the primary component of the cosmic rays. All this ratios are shown in Fig.3.4.

Another really important feature of atmospheric neutrinos is that for energies $\geq 1 \mathrm{GeV}$ neutrino fluxes should be isotropic, i.e. one should not observe upward-downward asymmetry. All this considerations become less and less accurate for higher energies but for energies between 1 and $100 \mathrm{GeV}$ the uncertainty on neutrino flux ratios $\left(\nu_{\mu}+\bar{\nu}_{\mu}\right) /\left(\nu_{e}+\bar{\nu}_{e}\right), \nu_{\mu} / \bar{\nu}_{\mu}$, and $\nu_{e} / \bar{\nu}_{e}$ are estimated to be within $2 \%, 5 \%$, and $5 \%$, respectively [19].

The first measurements of the atmospheric neutrino flux ratios were per- 


\begin{tabular}{lll}
\hline \hline & Reaction/Decay & Branching ratio (\%) \\
\hline$(\mathrm{A})$ & $p(n)+A \rightarrow \pi^{ \pm} X, K^{ \pm} X, K_{L} X$ & \\
$(\mathrm{~B} 1)$ & $\pi^{ \pm} \rightarrow \mu^{ \pm}+\nu_{\mu}\left(\bar{\nu}_{\mu}\right)$ & 99.9877 \\
$(\mathrm{~B} 2)$ & $\rightarrow e^{ \pm}+\nu_{e}\left(\bar{\nu}_{e}\right)$ & 0.0123 \\
$(\mathrm{C} 1)$ & $K^{ \pm} \rightarrow \mu^{ \pm}+\nu_{\mu}\left(\bar{\nu}_{\mu}\right)$ & 63.56 \\
$(\mathrm{C} 2)$ & $\rightarrow \pi^{0}+\mu^{ \pm}+\nu_{\mu}\left(\bar{\nu}_{\mu}\right)$ & 3.352 \\
$(\mathrm{C} 3)$ & $\rightarrow \pi^{0}+e^{ \pm}+\nu_{e}\left(\bar{\nu}_{e}\right)$ & 5.07 \\
$(\mathrm{D} 1)$ & $K_{L} \rightarrow \pi^{ \pm}+\mu^{\mp}+\bar{\nu}_{\mu}\left(\nu_{\mu}\right)$ & 27.04 \\
$(\mathrm{D} 2)$ & $\rightarrow \pi^{ \pm}+e^{\mp}+\bar{\nu}_{e}\left(\nu_{e}\right)$ & 40.55 \\
$(\mathrm{E})$ & $\mu^{ \pm} \rightarrow e^{ \pm}+\bar{\nu}_{\mu}\left(\nu_{\mu}\right)+\nu_{e}\left(\bar{\nu}_{e}\right)$ & 100 \\
\hline \hline
\end{tabular}

Table 3.1: Reactions and decays relevant to atmospheric neutrino and accelerator neutrino production. The first column shows the index of the reaction or decay, and the second column shows the reaction or decay channel. The third column shows the branching ratio. This table is taken from [8].

formed in the late 80 's by the Kamiokande collaboration. The result was an up/downward asymmetry just for $\nu_{\mu}$ neutrinos for energies $\sim 1 \mathrm{GeV}$ with a discrepancy of 2.8 standard deviations whereas the $\nu_{e}$ events confirmed the hypothesis that the flux was isotropic [20]. This result motivated the upgrade of the experiment named Super-Kamiokande. Super-Kamiokande (SK) is a cylindrical ring-imaging water Cherenkov detector measuring $39.2 \mathrm{~m}$ in diameter and $41 \mathrm{~m}$ in height located at a depth of $1000 \mathrm{~m}$ underground in the Mozumi mine in Kamioka, Japan. The detector is divided into an inner detector (ID) volume instrumented by 11146 inward facing 20 inch photomultiplier tubes (PMTs) providing a total photocathode coverage of $40 \%$. Surrounding this volume and separated from the wall of the detector by $2 \mathrm{~m}$ is an outer detector (OD) that utilizes 1885 outward facing 8 inch PMTs to veto environmental radiation and cosmic ray muons. A 22.5 kton fiducial volume is defined within the ID as the region offset from the IDPMTwall by $2 \mathrm{~m}$. Between 1996 and 2013 Super-K has accumulated 4220 days of atmospheric neutrino data corresponding to a $260 \mathrm{kton} \cdot$ year exposure. Already in $1998 \mathrm{SK}$ accomplished to measure up/downward $\nu_{\mu}$ asymmetry this time with a 6.2 standard deviation evidence. We show in Fig.3.5 the original slide presented by SK collaboration at the 18th International Conference on Neutrino Physics and Astrophysics (Neutrino 1998). This was explained by the fact that upward $\nu_{\mu}$ neutrinos, i.e. reaching the detector crossing the Earth, were changing flavour. Due to the fact that this effect was not visible for the $\nu_{e}$ flux it was interpreted that the $\nu_{\mu}$ were transforming into $\nu_{\tau}$. To verify this theory SK collaboration performed the same directional analysis for $\nu_{\tau}$-like events and actually find an excess of upward events confirming that atmospheric $\nu_{\mu}$ mix mainly with $\nu_{\tau}$. We show 


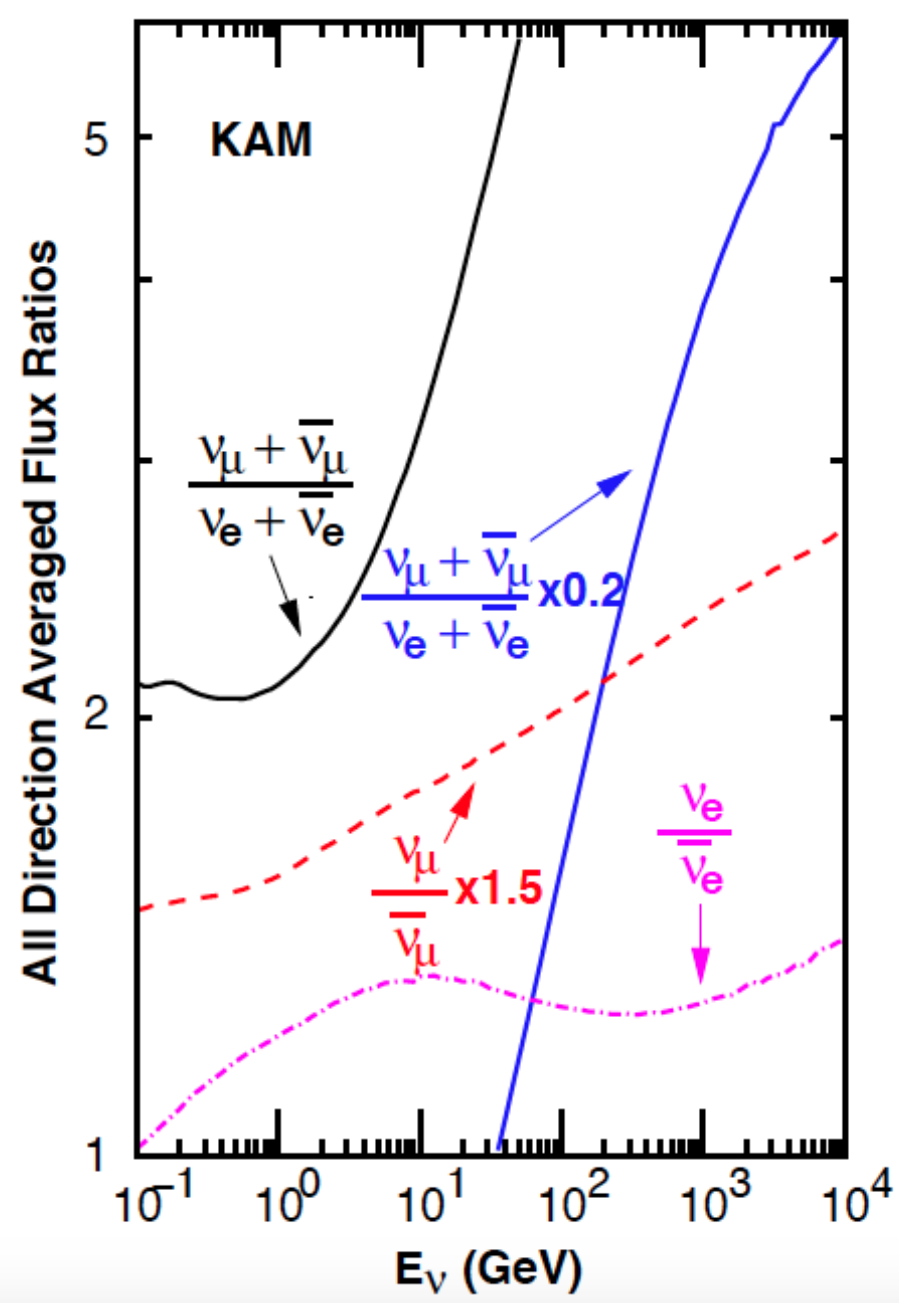

Figure 3.4: Neutrino flavour ratios calculated with the all-direction and oneyear averaged atmospheric neutrino fluxes at Kamioka. This figure is taken from [8].

this result in Fig.3.6 where experimental data are showed against the expected unoscillated flux calculation.

This phenomenon has been really well described by the oscillation probability in the simplified 2-flavour scenario

$$
P\left(\nu_{\mu} \rightarrow \nu_{\mu}\right)=1-\sin ^{2} 2 \theta_{23} \sin ^{2}\left(\frac{\Delta m_{32}^{2} L}{4 E}\right) .
$$

We report the SK results for the $\Delta m_{32}^{2}$ and $\theta_{23}$ parameters while taking $\theta_{13}$ and the 1-2 parameters as fixed in Fig.3.7. As one can see the best fit value for $\Delta m_{32}^{2}=2.6 \times 10^{-3} \mathrm{eV}^{2}$, so two order of magnitude of difference with respect to $\Delta m_{21}^{2}$, and $\sin ^{2} \theta_{23}=0.6$ but with a big uncertainty. This big degeneracy for the $\theta_{23}$ angle is present because the atmospheric experiments are sensitive just to $\sin ^{2} 2 \theta_{23}$ so unable to determine the octant to which $\theta_{23}$ belongs.

Other important experiments like MINOS, with different detection tech- 


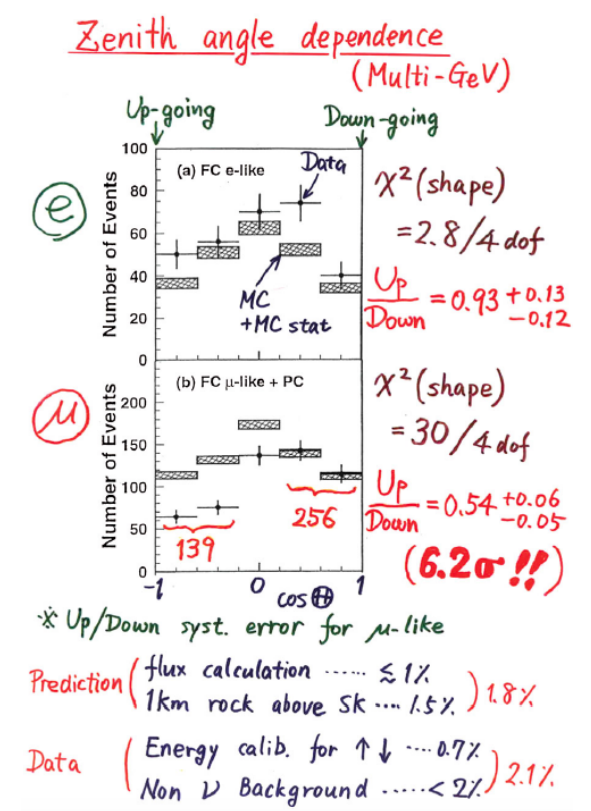

Figure 3.5: Zenith angle distributions for multi-GeV atmospheric neutrino events presented at the 18th International Conference on Neutrino Physics and Astrophysics (Neutrino'98) by the Super-Kamiokande collaboration. This figure is taken from [20].

niques confirmed the SK result. The flux model and the $\nu_{\mu}$ oscillation as been also verified at higher energies by the IceCube experiment. This is a neutrino telescope located near the south pole that even its primary objective is the detection of $\mathrm{PeV}$ neutrinos from astrophysical sources, it observes atmospheric neutrinos down to much lower energies. The detector is composed of 86 vertical strings drilled into the ice and bearing 60 optical modules spaced at 17 $\mathrm{m}$ intervals along $1 \mathrm{~km}$ of cable. IceCube has measured the atmospheric neutrino $\nu_{\mu}+\bar{\nu}_{\mu}$ energy spectrum using a zenith-averaged unfolding method on 18000 upward-going track-like events in the energy range of $100 \mathrm{GeV}$ to 400 $\mathrm{TeV}$. The other astrophysical neutrino telescope that also give some results on atmospheric neutrinos is ANTARES. This detector is located in the Mediterranean see and the detector consists of $450 \mathrm{~m} 12$ 'lines' separated by $65 \mathrm{~m}$ from each other. Each line is equipped with 25 optical modules (storeys) separated by a vertical distance of $14.5 \mathrm{~m}$.

The results of MINOS, IceCube and ANTARES are showed in Fig.3.8.

Atmospheric neutrino data motivated accelerator based longbaseline neutrino oscillation experiments. In a long-baseline neutrino oscillation experiment the neutrino flight length is fixed to a single value since the neutrino beam is produced by an accelerator and observed in a detector located at fixed distance away. It should also be noted that the beam in such experiments has a high $\nu_{\mu}$ (or $\bar{\nu}_{\mu}$ ) purity, while the atmospheric neutrino flux is a mixture of $\nu_{e}$, 


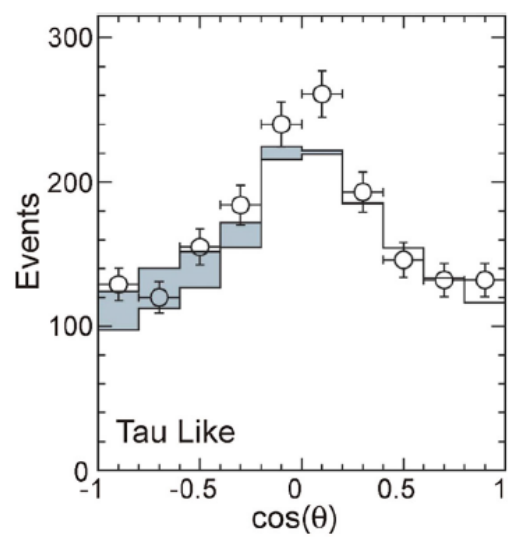

Figure 3.6: Zenith angle distributions for the $\tau$-like events selected from the data observed in Super-Kamiokande. Circles with error bars show the data. Solid histograms show the Monte Carlo prediction with $\nu_{\mu} \rightarrow \nu_{\tau}$ oscillations but without the charged current $\nu_{\tau}$ interactions. The gray histograms show the fit result including the $\nu_{\tau}$ interactions.. This figure is taken from [20].

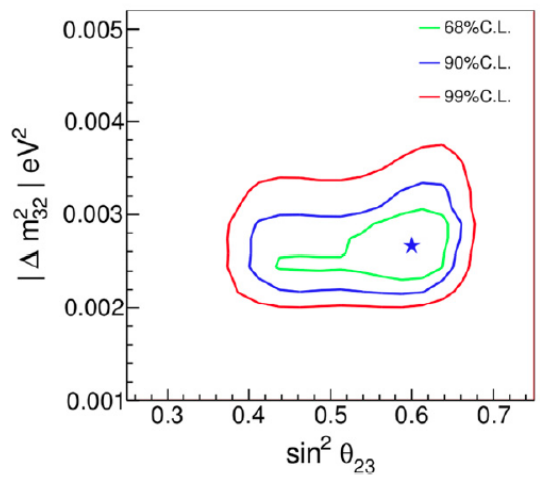

Figure 3.7: Results of the Super-K fit to three-neutrino oscillations. The plot shows the $\left|\Delta m_{32}^{2}\right|$ versus $\sin ^{2} \theta_{23}$ plane assuming an inverted mass hierarchy and a star indicates the best fit point. This figure is taken from [19].

$\bar{\nu}_{e}, \nu_{\mu}$ and $\bar{\nu}_{\mu}$. For these reasons long-baseline experiments are well suited to carry out precision measurements of the already explored 2-3 neutrino sector parameters and even more subtle measurement like the $\delta$ phase evaluation and the mass ordering identification.

Conventional method to produce neutrino beams at a high-energy proton accelerator facility is to guide an intense proton beam onto a nuclear target of $1 \sim 2$ interaction lengths. In the high-energy collisions, pions are dominantly produced, with kaons produced at an order of $10 \%$ of the pion production rate. Therefore, the dominant component of the accelerator neutrinos is the muon neutrino or muon antineutrino. So the oscillation channel that are explored are the $P\left(\nu_{\mu} \rightarrow \nu_{\mu}\right), P\left(\nu_{\mu} \rightarrow \nu_{e}\right)$ and their conjugate version. Due to the resolution of the first generation accelerator neutrino experiments and the fact 


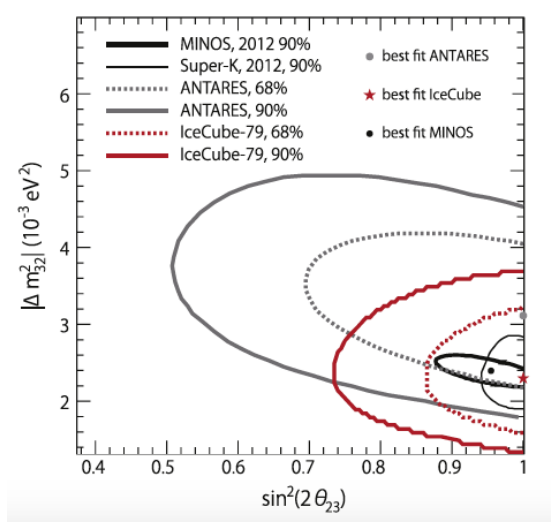

Figure 3.8: Allowed regions from the ANTARES and IceCube neutrino oscillation results compared with those of MINOS and Super- Kamiokande. This figure is taken from [19].

that $\Delta m_{21}^{2} /\left|\Delta m_{32}^{2}\right| \approx 0.03$ one can write these oscillation probability in the approximate form

$$
\begin{gathered}
P\left(\nu_{\mu} \rightarrow \nu_{\mu}\right)=1-\sin ^{2} 2 \theta_{23} \sin ^{2}\left(\frac{\Delta m_{32}^{2} L}{4 E}\right) \\
P\left(\nu_{\mu} \rightarrow \nu_{e}\right)=2 \sin ^{2} 2 \theta_{13} \sin ^{2} \theta_{23} \sin ^{2}\left(\frac{\Delta m_{32}^{2} L}{4 E}\right) .
\end{gathered}
$$

For the $\nu_{\mu} \rightarrow \nu_{e}$ channel one can see that accelerator experiment, even in this approximate form, can set a limit on the $\theta_{13}$ angle. We said just to get a limit instead of measure because its measurement suffer an uncertainty related, in particular, to the CPV phase $\delta$. Otherwise, we have reactor neutrino experiments that performed high precision measurement of $\theta_{13}$ (see next section) so that the next generation accelerator neutrino experiment will be able to evaluate the $\delta$ phase. We will show just the result of two of the most important accelerator neutrino experiments: T2K and NOvA.

The T2K experiment is the first off-axis long-baseline neutrino oscillation experiment. The baseline distance is $295 \mathrm{~km}$ between the J-PARC in Tokai, Japan and Super-Kamiokande. A narrow-band $\nu_{\mu}$ beam with a peak energy of $0.6 \mathrm{GeV}$, produced by $30 \mathrm{GeV}$ protons from the J-PARC Main Ring, is directed $2.5^{\circ}$ off-axis to SK. With this configuration, the $\nu_{\mu}$ beam is tuned to the first oscillation minimum of the $\nu_{\mu}$ survival probability. T2K started the first physics run in 2010. Already in $2013 \mathrm{~T} 2 \mathrm{~K}$ established $\nu_{\mu} \rightarrow \nu_{e}$ oscillation with $7.3 \sigma$ significance [16].

The NOvA experiment is an off-axis long-baseline neutrino oscillation experiment using the the NuMI medium-energy beam. Its detectors are positioned $14.6 \mathrm{mrad}$ off-axis. With this configuration, the neutrino beam has a narrow spectrum which peaks at around $2 \mathrm{GeV}$. The 14 kton total active mass far 
detector is located on the surface at Ash River, Minnesota, $810 \mathrm{~km}$ from the production target. The 193 ton total active mass near detector is located $100 \mathrm{~m}$ underground at Fermilab, approximately $1 \mathrm{~km}$ from the target. Both detectors are fine-grained tracking calorimeters consisting of arrays of PVC cells filled with liquid scintillator. NOvA started physics run in 2014.

Both of them showed their last results in the last International Conference on Neutrino Physics and Astrophysics (Neutrino 2018). We show in Fig.3.9 the results for T2K [21] and in Fig.3.10 the results for NOvA [22].
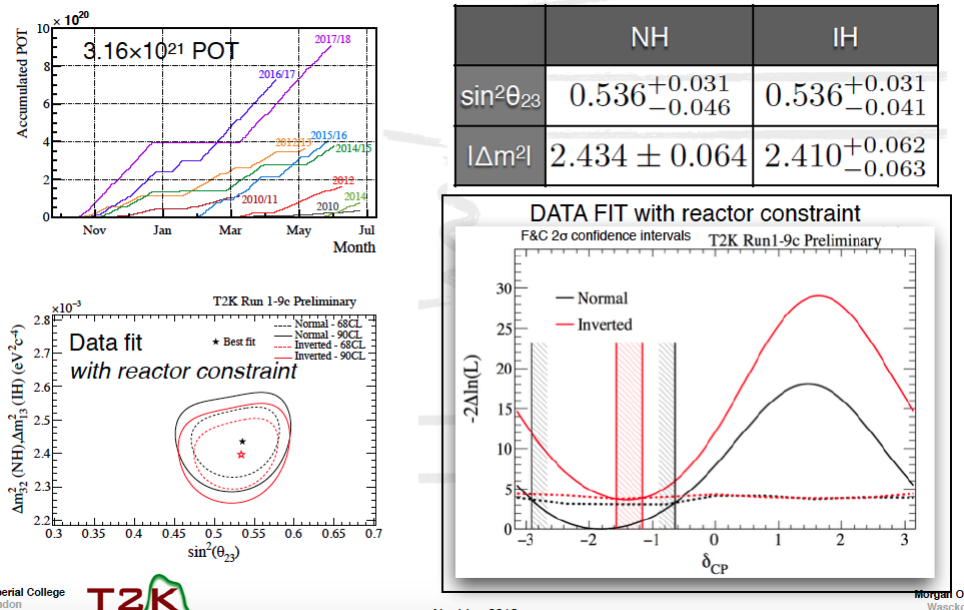

Figure 3.9: Here we show the most recent results of the T2K collaboration. In this figure we have the allowed region for $\Delta m_{32}^{2}$ and $\theta_{23}$ for both IO and $\mathrm{NO}$ ordering at 1 and $2-\sigma$ confidence level (bottom-left), and the $2-\sigma$ allowed region for the $\mathrm{CP} \delta$ values (bottom-right). We also show the cumulative statistics for the different run (top-left) and the best fit values for $\Delta m_{32}^{2}$ and $\theta_{23}$ in IO and NO hypothesis. This figure is taken from [21].

The next generation accelerator experiment, e.g. DUNE [23] and T2HK/T2HKK [16, 24], will try to test the neutrino mixing scheme beyond the 2-flavour approximated model we showed before. They will be sensible to the modifications in the $\nu_{\mu} \rightarrow \nu_{e}$ channel (and its conjugate version) due to the different values of $\delta$ and to the mass ordering. They will be really long baseline experiments $(\sim 1000 \mathrm{~km})$ so that they will place in the second maximum of the oscillation probability. The non negligible matter effect will be fundamental in the selection of the mass ordering and the determination of the exact value of the $\delta$ phase [8]. 


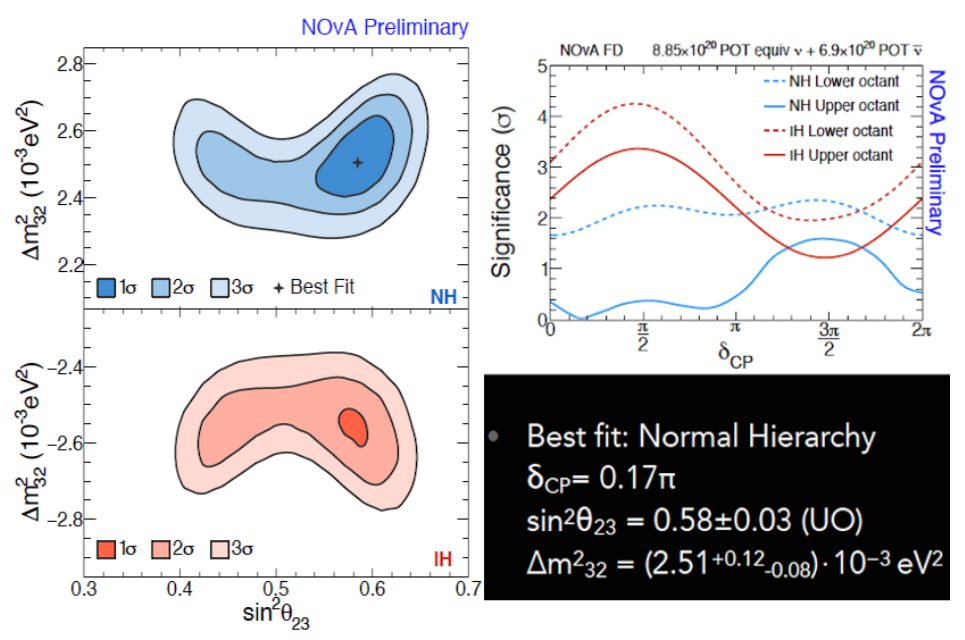

Figure 3.10: Here we show the most recent results of the NOvA collaboration. In this figure we have the allowed region for $\Delta m_{32}^{2}$ and $\theta_{23}$ for both IO and NO ordering at 1 and $2 \sigma$ confidence level (left), and the confidence level of the allowed region for the $\mathrm{CP} \delta$ values (top-right). We also show the the best fit values for $\Delta m_{32}^{2}$ and $\theta_{23}$ for the best fit NO hypothesis (bottom-right). This figure is taken from [22].

\section{3}

\section{Reactor Neutrinos}

Reactor neutrino studies have been historically the ones that proved the existence of neutrinos. In 1956 Frederick Reines and Clyde Cowan performed the pioneering experiment at the Hanford and Savanah River nuclear reactors to detect neutrinos directly via the IBD reaction suggested by Bethe and Peierls [25]. Since then, no flavour oscillation was detected in reactor neutrinos experiment until 2002 when the Kamland collaboration announced the result we showed in the first section of this chapter. After the solar and atmospheric neutrinos results in 2006 three experiments Daya Bay, Double Chooz and RENO were proposed to probe $\theta_{13}$. All these experiments have really similar neutrino sources, detection techniques and follow the same phenomenological strategy to test the 1-3 neutrino sector. We summarize the neutrino source power, the detector locations and the detector masses in Table 3.2.

A detailed estimation of the neutrino flux coming from a nuclear reactor core is a really complicated topic and we present just a rough calculation. In nuclear reactors, power is generated mainly by nuclear fission of four heavy isotopes, ${ }^{235} \mathrm{U},{ }^{238} \mathrm{U},{ }^{239} \mathrm{Pu}$, and ${ }^{241} \mathrm{Pu}$. These isotopes account for more than $99 \%$ of fissions in the reactor core. $\beta$-decays of fission products produce almost pure $\bar{\nu}_{e}$ flux. The rate of $\nu_{e}$ production is less than $10^{-5}$ of the rate of $\bar{\nu}_{e}$ production. As the daughter isotopes of each fission undergo $6 \beta$-decays on average, 6 electron antineutrinos are emitted per fission. The thermal power 


\begin{tabular}{l|l|l|l}
\hline \hline Experiment & Power $\left(G W_{t h}\right)$ & Baseline $(\mathrm{m})$ & Mass (tons) \\
\hline \multirow{2}{*}{ Daya Bay } & & & \\
& 17.4 & 360 & $2 \times 20$ \\
& & 500 & $2 \times 20$ \\
& & 1580 & $4 \times 20$ \\
\hline RENO & 16.8 & 290 & 16 \\
& & 1380 & 16 \\
\hline Double Chooz & 8.5 & 400 & 8 \\
& & 1050 & 8 \\
\hline \hline
\end{tabular}

Table 3.2: Key parameters of the three experiment we are referring to. Data from $[25]$.

outputs of nuclear power reactors are usually quoted in thermal GW, GW $\mathrm{GW}_{t h}$. The effective energy released per fission is $\sim 200 \mathrm{MeV}$. Therefore, with 1 $\mathrm{GW}_{\text {th }}$ output, $\sim 2 \times 10^{20}$ electron antineutrinos are produced per second and emitted isotropically [25]. In Fig.3.11 we show the $\bar{\nu}_{e}$ energy spectrum of the convolution of the flux with the IBD cross section for each isotope.

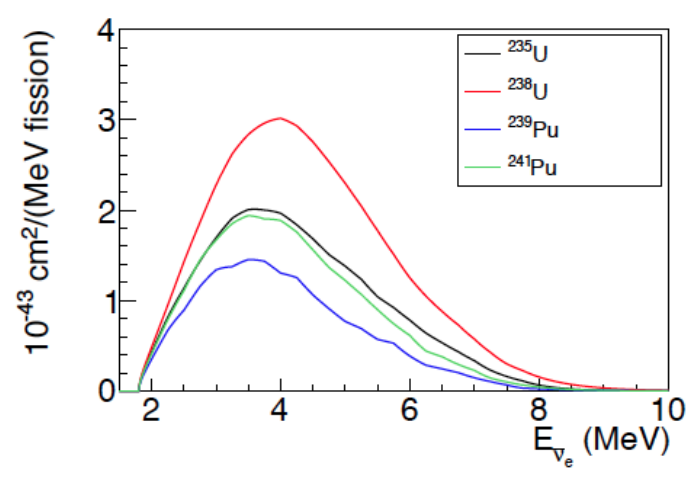

Figure 3.11: Inverse beta decay yields from the convolution of the IBD cross section and the antineutrino spectra for ${ }^{235} \mathrm{U},{ }^{238} \mathrm{U},{ }^{239} \mathrm{Pu}$, and ${ }^{241} \mathrm{Pu}$. This figure is taken from [25].

The description of an IBD event is illustrated in Fig.3.12. An event is indicated by a pair of coincident signals consisting of i) a prompt signal induced by positron ionization and annihilation inside the detector; and ii) a delayed signal produced by the neutron captured on a proton or a nucleus (such as Gd). Because of time correlation, IBD can be clearly distinguished from radioactive backgrounds. It is important to remark that this technique has been extensively used and slightly modified since 1956 and it is still used today.

The oscillation probability channel that is investigated in all the reactor neutrino experiments cited before is $P\left(\bar{\nu}_{e} \rightarrow \bar{\nu}_{e}\right)$. The full three flavour version of this survival probability is 


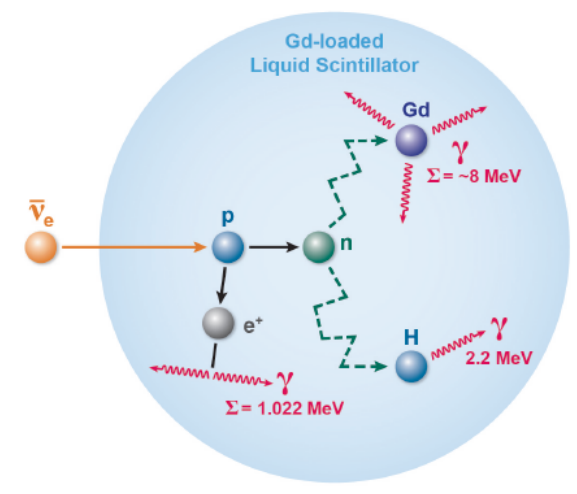

Figure 3.12: Pictorial representation of the IBD detection in a Gd-loaded liquid scintillator. The $\bar{\nu}_{e}$ interacts with a free proton producing a $e^{+}$. The annihilation of the positron represent the prompt signal otherwise the recoil neutron capture on Gd (or H) represent the delayed signal. This figure is taken from [25].

$$
P\left(\bar{\nu}_{e} \rightarrow \bar{\nu}_{e}\right)=1-\sin ^{2} 2 \theta_{13}\left(c_{12}^{2} \sin ^{2} \Delta_{31}+s_{12}^{2} \sin ^{2} \Delta_{32}\right)-c_{13}^{4} \sin ^{2} 2 \theta_{12} \sin ^{2} \Delta_{21},
$$

where $\Delta_{i j}=\frac{\Delta m_{i j}^{2} L}{4 E}$. To measure $\theta_{13}$ one has to maximize the term proportional to $\sin ^{2} 2 \theta_{13}$. Because the $2 \lesssim \bar{\nu}_{e} \lesssim 8 \mathrm{MeV}$ one has to consider baselines of the order of $\sim 1 \mathrm{~km}$ as one can see in Table 3.2. In this configuration the part that is proportional to $\sin ^{2} \Delta_{21}$ can be averaged out due to $\Delta m_{21}^{2} /\left|\Delta m_{32}^{2}\right| \approx 0.03$. Using this peculiar neutrino mass splitting properties, it is possible to rewrite Eq.(3-9) defining an effective atmospheric mass difference

$$
\Delta_{\eta}=\Delta_{31}-\eta \Delta_{21}=\Delta_{32}+(1-\eta) \Delta_{21},
$$

that after some calculations gives [26]

$$
\begin{aligned}
1-P\left(\nu_{\alpha} \rightarrow \nu_{\alpha}\right) & =4\left|U_{\alpha 3}\right|^{2}\left(1-\left|U_{\alpha 3}\right|^{2}\right)\left[\sin ^{2} \Delta_{\eta}\right. \\
& +\left\{r_{1} \sin ^{2}\left(\eta \Delta_{21}\right)+r_{2} \sin ^{2}\left((1-\eta) \Delta_{21}\right)\right\} \cos 2 \Delta_{\eta} \\
& \left.+\frac{1}{2}\left\{r_{1} \sin \left(2 \eta \Delta_{21}\right)+r_{2} \sin \left(2(1-\eta) \Delta_{21}\right)\right\} \sin 2 \Delta_{\eta}\right] \\
& +4\left|U_{\alpha 2}\right|^{2}\left|U_{\alpha 1}\right|^{2} \sin ^{2} \Delta_{21}
\end{aligned}
$$

where

$$
r_{1}=\frac{\left|U_{\alpha 1}\right|^{2}}{\left|U_{\alpha 2}\right|^{2}+\left|U_{\alpha 1}\right|^{2}} \quad r_{2}=\frac{\left|U_{\alpha 2}\right|^{2}}{\left|U_{\alpha 2}\right|^{2}+\left|U_{\alpha 1}\right|^{2}} .
$$

From the minimization of the $\cos 2 \Delta_{\eta}$ and $\sin 2 \Delta_{\eta}$ coefficients one can find that $\eta \approx r_{2}$ that allows to redefine the effective atmospheric mass splitting in the form

$$
\Delta m_{\alpha \alpha}^{2}=r_{1} \Delta m_{31}^{2}+r_{2} \Delta m_{32}^{2} .
$$

As one can see this effective mass depends on the flavour $\alpha$, i.e. depends on the survival probability channel we are considering. In our case, reactor experiment 
are able to measure $\Delta m_{e e}^{2}$

$$
\Delta m_{e e}^{2}=c_{12}^{2} \Delta m_{31}^{2}+s_{12}^{2} \Delta m_{32}^{2},
$$

otherwise accelerator and atmospheric experiments can measure $\Delta m_{\mu \mu}^{2}$

$$
\Delta m_{\mu \mu}^{2}=s_{12}^{2} \Delta m_{31}^{2}+c_{12}^{2} \Delta m_{32}^{2}+\cos \delta s_{13} \sin 2 \theta_{12} \tan \theta_{23} \Delta m_{21}^{2} \text {. }
$$

The most evident physical consequence of this approach is that in principle with high precision measurement of these two effective mass splitting one can determine the mass ordering and also the $\delta$ phase. This appear more evident if we subtract one mass to the other [26]

$$
\left|\Delta m_{e e}^{2}\right|-\left|\Delta m_{\mu \mu}^{2}\right|= \pm \Delta m_{21}^{2}\left(\cos 2 \theta_{12}-\cos \delta s_{13} \sin 2 \theta_{12} \tan \theta_{23}\right) .
$$

The determination of the mass ordering and the $\delta$ phase by this method will be the aim of the next generation reactor neutrino experiment like JUNO [17]. The present stage of our knowledge is resumed in Fig.3.13. On the left side of the figure we have the $\theta_{13}$ measurement of Daya Bay, RENO and Double Chooz. As one can see the best $\theta_{13}$ estimation belongs to the Daya Bay experiment, that in 2012 published its results $\sin ^{2} \theta_{13}=0.0841 \pm 0.0027$ (stat.) \pm 0.0019 (syst.) and $\left|\Delta m_{e e}^{2}\right|=2.50 \pm 0.06$ (stat) $\times 10^{-3} \mathrm{eV}^{2}$. On the right side of Fig.3.13 instead we have a comparison of the results of accelerator, atmospheric and reactor neutrino experiment for the value of $\Delta m_{32}^{2}$.
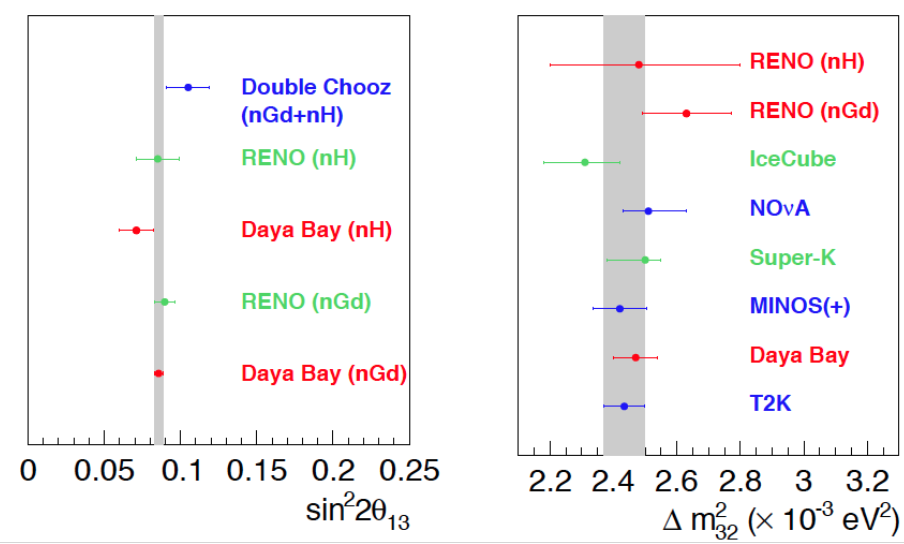

Figure 3.13: Global result of $\theta_{13}$ (left) and $\Delta m_{32}^{2}$ (right) taken from the results presented at the Neutrino 2018 conference. For $\Delta m_{32}^{2}$ NO hypothesis is considered only. This figure is taken from [25]. 


\section{4}

\section{Oscillation Parameter: Global Analysis}

The experimental data used in [27] to perform the global fit are:

- Solar Data: this set is composed by the radiochemical experiments Chlorine, Gallex/GNO and SAGE, the results of the four SuperKamiokande phases, three phases of SNO and the two phases of Borexino.

- Long Baseline Data: the final energy spectrum of accelerator experiment MINOS, T2K and NO $\nu \mathrm{A}$ in the $\nu_{\mu} / \bar{\nu}_{\mu}$ disappearance and $\nu_{e} / \bar{\nu}_{e}$ appearance channels.

- Reactor Data: in this set we have the long baseline reactor data from KamLAND, the medium baseline reactor data from $\mathrm{CHOOZ}$ and Palo Verde as well as the partial results (2016) of Double-Chooz, Daya-Bay and RENO.

In Fig. 3.14 we show the global analysis for all the 6 parameters in the case of NO and IO. All the best fit values for NO and IO cases are reported in Table 3.3.

\begin{tabular}{l|l|l}
\hline \hline & NO bf $\pm 1-\sigma$ & IO bf $\pm 1-\sigma$ \\
\hline & & \\
$\sin ^{2} \theta_{12}$ & $0.306_{-0.012}^{+0.012}$ & $0.306_{-0.012}^{+0.012}$ \\
$\sin ^{2} \theta_{23}$ & $0.441_{-0.022}^{+0.027}$ & $0.587_{-0.024}^{+0.020}$ \\
$\sin ^{2} \theta_{13}$ & $0.02166_{-0.00075}^{+0.00075}$ & $0.02179_{-0.00076}^{+0.00076}$ \\
$\frac{\Delta m_{21}^{2}}{10^{-5} \mathrm{eV}^{2}}$ & $7.5_{-0.17}^{+0.19}$ & $7.5_{-0.17}^{+0.19}$ \\
$\frac{\Delta m_{31}^{2}}{10^{-3} \mathrm{eV}^{2}}$ & $2.524_{-0.040}^{+0.039}$ & $2.514_{-0.041}^{+0.038}$ \\
\hline \hline
\end{tabular}

Table 3.3: Oscillation parameter table from Maltoni et al.[27].

With reference to Fig.3.14, there are three parameter that has been determined with reasonably good accuracy and they are $\theta_{12}, \theta_{13}, \Delta m_{21}^{2}$. Their best fit values comes mostly from solar neutrino data $\left(\theta_{12}, \Delta m_{21}^{2}\right)$ and reactor neutrino data $\left(\theta_{13}\right)$ and, as physically expected, they are almost independent of the mass ordering with NO slightly favoured over IO.

The other three parameter $\left(\theta_{23}, \delta_{C P}\right.$ and the mass ordering) suffer a much bigger uncertainties mainly because of the presence of degeneracy among oscillation parameters. This will be the focus of the new generation experiments like JUNO [17], DUNE [23] and HK [16]. For what it concerns $\theta_{23}$ one can see that the $\chi^{2}$ function present a two fold shape. This is the manifestation of the so called octant degeneracy. This degeneracy is caused by the fact that 
the accelerator (atmospheric) experiments in the $\nu_{\mu} \rightarrow \nu_{\mu}$ channel are mainly sensible to $\sin ^{2} 2 \theta_{23}$ and this leads, at first approximation, ignoring $\theta_{13}$, to the following 2 degenerate solutions, $\sin ^{2} \theta_{23}=\left(1 \pm \sqrt{1-\sin ^{2} 2 \theta_{23}}\right) / 2$ [28]. We have that for NO $\theta_{23}<45^{\circ}$ is favoured at $\propto 1 \sigma$ and for IO case $\theta_{23}>45^{\circ}$ is favoured at $\propto 1.8 \sigma$ [27]. In order to use a more conservative approach, we will consider three different best fit values for this parameter: one will be addressed as the low case $s_{23}^{2}=0.441 \pm 0.025$, one as the mid case $s_{23}^{2}=0.5 \pm 0.042$ and one as the high case $s_{23}^{2}=0.587 \pm 0.022$. For the $\delta$ phase the analysis gives us, in both NO and IO cases, a "hint" of a $270^{\circ}$. 

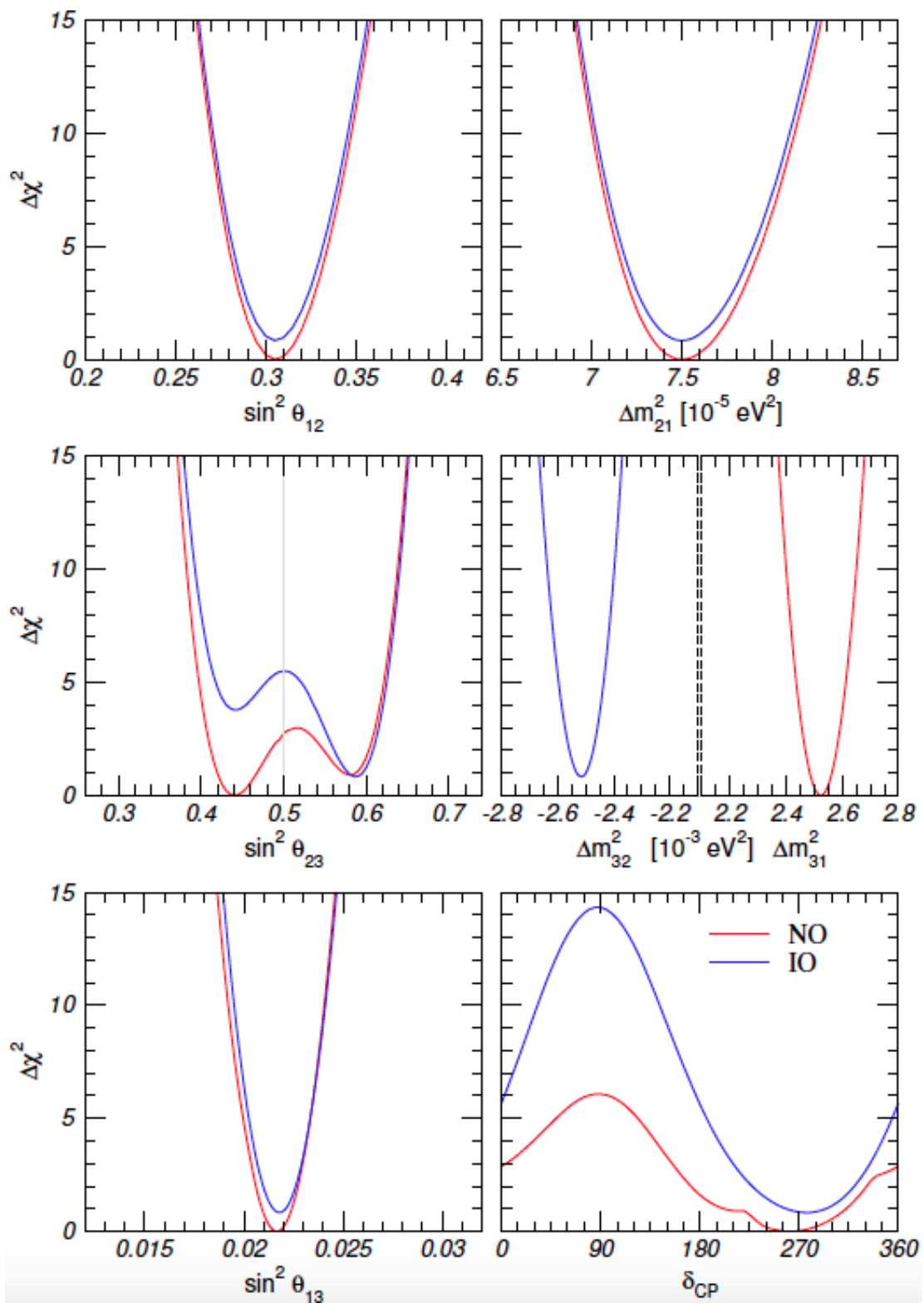

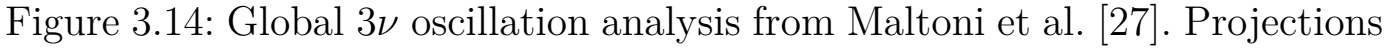
of the $\chi^{2}$ function onto the parameters $\Delta m_{21}^{2}$ (solar), $\Delta m_{31}^{2} / \Delta m_{32}^{2}$ (atmospheric), $\sin ^{2} \theta_{i j}$, and $\delta$, for Normal Ordering (blue) and Inverted Ordering (red). In each panel all the undisplayed parameters are marginalized. 


\section{A Novel Method to Measure the Leptonic CP-asymmetry: Experimental Set Up}

The measurement of the leptonic $\mathrm{CP}$ violation is one of the most important research topics in particle physics. The $\delta$ phase, also called Dirac phase, that regulates the magnitude of this phenomenon is the one of the last missing parameter ${ }^{1}$ to completely pin down the PMNS lepton mixing matrix. If the seek of "completeness" is not enough to justify the interest in this peculiar phenomenon it is actually really important for different theoretical reasons.

The exact magnitude of this quantity can shed a light, for example, on what is called leptogenesis, that is a process that could explain the present baryon-antibaryon asymmetry of the universe [29]. It will be able to select a number of neutrino mass generating mechanism [30].

Until now no experiment was realized with the capability to measure this asymmetry with high significance, but after 2012 when $\theta_{13}$ angle was discovered to be non-zero [31], a series of new proposal came out.

Neutrino flavour oscillation measurement will be the way pursued to observe this violation. From the theory we know that it is only possible to see CP asymmetry when the neutrino change from one flavour to another. For experimental reason the channels that all the experiments are considering is the $\nu_{\mu} \rightarrow \nu_{e}$ and its conjugate $\bar{\nu}_{\mu} \rightarrow \bar{\nu}_{e}$. Experiments like DUNE [23] and T2HK [16], and its possible extension in Korea T2HKK [24] are long baseline (LB) neutrino detectors whose beams come respectively from Fermilab $(1300 \mathrm{~km})$ and form the J-PARK facilities $(295 \mathrm{~km}$ for T2HK and $1100 \mathrm{~km}$ for T2HKK). The beam of $\nu_{\mu}\left(\bar{\nu}_{\mu}\right)$ are produced by sending protons on carbon targets generating $\pi^{ \pm}$that pass through the decay chain

$$
\begin{aligned}
\pi^{+} \rightarrow & \mu^{+}+\nu_{\mu} & \pi^{-} \rightarrow & \mu^{-}+\bar{\nu}_{\mu} \\
& \downarrow & & \downarrow \\
& e^{+}+\nu_{e}+\bar{\nu}_{\mu} & & e^{-}+\bar{\nu}_{e}+\nu_{\mu}
\end{aligned}
$$

These new generation experiment, even if they are really different when it comes to detection process, energy reconstruction, etc., they will both mea-

\footnotetext{
${ }^{1}$ The other unknowns are the mass ordering and the octant of the $\theta_{23}$ angle.
} 
sure $\delta_{C P}$ by looking at appearance probability differences between $\nu_{e}$ coming from the $\nu_{\mu}$ highlighted in the decay chain and its conjugate counterpart. The main drawbacks are: one is that neutrino beams has to work in different configurations to produce $\nu_{\mu}$ and $\bar{\nu}_{\mu}$, which implies large systematic uncertainties at the detection level; the other is that the flux has to be known with high accuracy due to the fact that in LB experiments the matter effect can mimic the $\mathrm{CP}$ violation.

Another method has been explored to overcome these issues and was first theoretically proposed by Minakata et al. [32]. The idea is to use only a $\nu_{\mu}$ beam and compare the $\nu_{e}$ appearance in two or three identical detectors placed at specific locations in a way to maximize the $\mathrm{CP}$ effect and to overcome the flux uncertainty. In practice what has been proposed by Conrad et al. [33] is to use a single detector that will detect neutrinos coming from three different cyclotrons disposed at $1.5 \mathrm{~km}$ (near), $8 \mathrm{~km}$ (middle) and $20 \mathrm{~km}$ (far). One of this method weak point is the use of multiple facilities, that again, introduces systematic errors.

The novel method we are going to study in this chapter utilizes an unseen framework. It will be based on a flux of $\nu_{\mu}$ and $\bar{\nu}_{\mu}$ coming from a Decay At Rest (DAR) $\pi^{+}$

$$
\begin{aligned}
\pi^{+} \rightarrow & \mu^{+}+\nu_{\mu} \\
& \downarrow \\
& e^{+}+\nu_{e}+\bar{\nu}_{\mu}
\end{aligned}
$$

and on the simultaneous detection of $\nu_{e}$ and $\bar{\nu}_{e}$ coming from the oscillation of $\nu_{\mu}$ and $\bar{\nu}_{\mu}$ highlighted. In this way it is possible to utilize the whole decay chain and the neutrino and antineutrino fluxes are necessarily isotropical and equal. The detection will be performed using opaque Liquid Scintillator (LS) that allows $e^{+} e^{-}$identification. This identification is also strongly enhanced by the different signature of $\nu_{e}$ and $\bar{\nu}_{e}$ interactions. Indeed, for the $\nu_{\mu} \rightarrow \nu_{e}$ we have a monochromatic spectrum because we are selecting DAR $\pi^{+}$. The $\nu_{\mu}$ energy is about $30 \mathrm{MeV}$ because we have that the pion and the muon rest mass are roughly $140 \mathrm{MeV}$ and $105.6 \mathrm{MeV}$ respectively. The $\bar{\nu}_{\mu} \rightarrow \bar{\nu}_{e}$ spectrum instead has a continuous character because it is a product of a 3-body decay and the energy range spans from 1.8 to $52.8 \mathrm{MeV}$. The detector will be placed at first oscillation maximum, that for the range of energy considered, is around $16 \mathrm{~km}$ so that we can neglect the matter effect.

This work will focus on the phenomenological potentials of this new framework. 
Chapter 4. A Novel Method to Measure the Leptonic CP-asymmetry:

Experimental Set Up

\section{1}

\section{Neutrino Coming From $\pi^{+}$Decay At Rest Chain}

Our neutrino source is supposed to be coming from a cyclotron, or in general, an accelerator beam dump that target high energy protons with alternate intensity. This will produce an intense and isotropic neutrino flux coming from $\pi^{+}$DAR. We expect to have $4 \cdot 10^{22}$ neutrinos per flavour per year [33]. We will not get into more details about the pion production because the experiment is still in $R \& D$ phase so there is no actual accelerator to refer to.

What we know really well is the process that generates our neutrinos. So as we have seen in the introduction we will be interested in $\nu_{\mu}$ coming from the $\pi^{+}$DAR and $\bar{\nu}_{\mu}$ coming from $\mu^{+}$decay. Let us start from the $\pi^{+}$case. First of all, the $\pi^{+}$is a spin-0 pseudoscalar boson with mass $m_{\pi} \approx 140 \mathrm{MeV}$. It has a lifetime of $26 \mathrm{~ns}$ and it decays by the 2-body decay mode

$$
\pi^{+} \rightarrow \mu^{+}+\nu_{\mu} \quad(99.99 \%)
$$

$$
\pi^{+} \rightarrow e^{+}+\nu_{e} \quad(0.012 \%),
$$

with the values in parenthesis being the channel's branching ratio [1]. The decay tree level Feynman diagram is showed in Fig.4.1. For this process being a 2-body decay the energy and the momentum carried by the neutrino and the lepton are fixed by the relations

$$
E_{\nu}=\frac{m_{\pi}^{2}-m_{l}^{2}}{2 m_{\pi}} \quad E_{l}=\frac{m_{\pi}^{2}+m_{l}^{2}}{2 m_{\pi}} \quad|\vec{p}|=\frac{m_{\pi}^{2}-m_{l}^{2}}{2 m_{\pi}},
$$

where neutrino are assumed massless in the range of energy we are referring to (hundreds of $\mathrm{MeV}$ ).

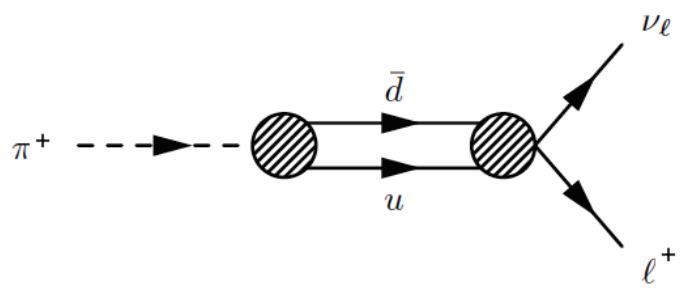

(a)

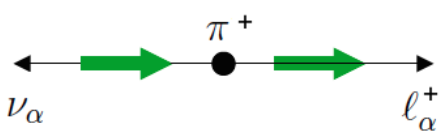

(b)

Figure 4.1: a)Tree level first order Feynman diagrams representation of the $\pi^{+}$decay. b) A representation of the final excluded helicity state for the limit $m_{l}=0$. In this case one can just have a left-handed helicity neutrino and a right-handed helicity anti-lepton that violate the spin angular momentum conservation. The green arrows represents the particle spin and the thin black ones representing the momentum of the particles. Figure edited from [2] 
The effective low energy amplitude of the process is

$$
A_{\pi^{+} \rightarrow l^{+} \nu_{l}}=-\mathrm{i} \frac{G_{F}}{\sqrt{2}} V_{d u} \bar{v}_{d} \gamma^{\mu}\left(1-\gamma^{5}\right) u_{u} \bar{v}_{\mu^{+}} \gamma_{\mu}\left(1-\gamma^{5}\right) u_{\nu_{\mu}} .
$$

We have seen in the first chapter that to taking into account the fact that $u$ and $\bar{d}$ are a bounded system in the strong interaction potential we must rewrite the quark part of the amplitude as

$$
\bar{v}_{d} \gamma^{\mu}\left(1-\gamma^{5}\right) u_{u} \bar{v}_{\mu^{+}} \gamma_{\mu}\left(1-\gamma^{5}\right) u_{\nu_{\mu}} \rightarrow \frac{1}{m_{\pi}}\left\langle 0\left|\bar{d} \gamma^{\mu}\left(1-\gamma^{5}\right) u\right| \pi^{+}\left(p_{\pi}=m_{\pi}\right)\right\rangle,
$$

where the factor $1 / m_{\pi}$ serves to keep the dimensions right (the current has dimensions $E^{3}$ and the pion state has dimension $E^{-1}$ ).

$$
\left\langle 0\left|\bar{d} \gamma^{\mu}\left(1-\gamma^{5}\right) u\right| \pi^{+}\left(p_{\pi}=m_{\pi}\right)\right\rangle=\mathrm{i} f_{\pi} m_{\pi},
$$

where $f_{\pi}$ being the structure function of the pion, which corresponds to the overlapping density of the wave function of the quarks in the pion. So what is important is that the amplitude, using the relation (4-3), is proportional to the mass of the produced lepton. The decay rate final formula reads

$$
\Gamma_{\pi^{+} \rightarrow l^{+} \nu_{l}}=\frac{G_{F}^{2}}{8} f_{\pi}^{2}\left|V_{d u}\right|^{2} m_{\pi} m_{l}^{2}\left(1-\frac{m_{l}^{2}}{m_{\pi}^{2}}\right)^{2}
$$

If we compare the electron and muon decay rate we will obtain

$$
\frac{\Gamma_{\pi^{+} \rightarrow e^{+} \nu_{e}}}{\Gamma_{\pi^{+} \rightarrow \mu^{+} \nu_{\mu}}}=\left(\frac{m_{e}^{2}}{m_{\mu}^{2}}\right)\left(\frac{m_{\pi}^{2}-m_{e}^{2}}{m_{\pi}^{2}-m_{\mu}^{2}}\right)^{2}=1.28 \times 10^{-4},
$$

that agrees with the rates we showed before. This electron suppression is also known as helicity suppression. For massless particle helicity (the projection of the z-component of the spin on the momentum direction) coincides with chirality (characteristic that differentiates left handed and right handed fermions). As we showed in the first chapter, the weak interaction select just neutrinos left-handed chiral state and anti-neutrinos right-handed chiral state. So as shown in Fig.4-3 if in the final state we cannot have a right-handed helicity anti-lepton, because the total final state spin must be equal to the initial one i.e. zero. The probability to have a positron or an anti-muon in a left-handed helicity state is

$$
P_{e^{+}}=\frac{1-\beta_{e}}{2}=\frac{m_{e}^{2}}{m_{\pi}^{2}-m_{e}^{2}} \approx 1.3 \times 10^{-5}, \quad P_{\mu+}=\frac{1-\beta_{e}}{2}=\frac{m_{\mu}^{2}}{m_{\pi}^{2}-m_{\mu}^{2}} \approx 0.36,
$$

that confirms the rate we have already calculated. To resume, we have that the $\pi^{+}$decays totally in the muon channel and the $\nu_{\mu}$ energy is fixed by the kinematics expression (4-3) 


$$
E_{\nu_{\mu}}=\left|\vec{p}_{\nu_{\mu}}\right|=\frac{m_{\pi}^{2}-m_{\mu}^{2}}{2 m_{\pi}}=\frac{140^{2}-106^{2}}{2 \cdot 140}[\mathrm{MeV}] \approx 30 \mathrm{MeV}
$$

Let us now look at the $\mu^{+}$decay. This is a 3-body decay where the product particles are considered massless with respect to the $\mu^{+}$. From Fig.4.2 we can write down the amplitude

$$
A=-\mathrm{i} G_{F} 2 \sqrt{2}\left[\bar{v}_{\mu^{+}} \gamma^{\mu}\left(1-\gamma^{5}\right) v_{\bar{\nu}_{\mu}}\right]\left[\bar{u}_{\nu_{e}} \gamma_{\mu}\left(1-\gamma^{5}\right) v_{e^{+}}\right] .
$$

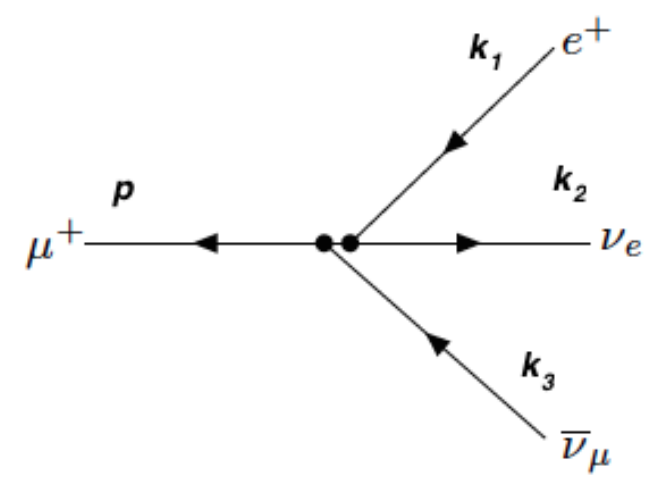

Figure 4.2: Tree level Feynman diagram of the $\mu^{+}$decay where the particles 4-momenta are indicated with the letters $p, k_{1}, k_{2}$ and $k_{3}$.

The differential decay rate is proportional to the square module of the amplitude

$$
\frac{\mathrm{d} \Gamma}{\mathrm{d} E_{1} \mathrm{~d} E_{2}}=\frac{1}{64 \pi^{3} m_{\mu^{+}}} \sum_{\text {spin }}|A|^{2}
$$

where the indices 1 and 2 are arbitrarily chosen as shown in Fig.4.2. Following the same convention, after a long but straightforward calculation the square module of the amplitude summed over all the final spin states is

$$
\sum_{\text {spin }}|A|^{2}=64 G_{F}^{2}\left(p^{\mu^{+}} \cdot k_{2}^{\nu_{e}}\right)\left(k_{3}^{\bar{\nu}_{\mu}} \cdot k_{1}^{e^{+}}\right),
$$

where $p, k_{1}, k_{2}$ and $k_{3}$ are the 4-momenta of all the particles involved. If one writes this quantity in the $\mu^{+}$rest frame and chooses the direction of emission of $\bar{\nu}_{e}$ being $z$ it becomes

$$
\begin{gathered}
p=\left(m_{\mu}, 0,0,0\right), \quad k_{2}=\left(E_{\nu_{e}}, 0,0, E_{\nu_{e}}\right), \\
\left(p^{\mu^{+}} \cdot k_{2}^{\nu_{e}}\right)=m_{\mu} E_{\nu_{e}}, \\
\left(k_{3}^{\bar{\nu}_{\mu}} \cdot k_{1}^{e^{+}}\right)=\frac{1}{2}\left(\left(k_{3}+k_{1}\right)^{2}-k_{3}^{2}-k_{1}^{2}\right)=\frac{1}{2}\left(\left(p-k_{2}\right)^{2}-0-0\right)=\frac{1}{2}\left(m_{\mu}^{2}-2 m_{\mu} E_{\nu_{e}}\right),
\end{gathered}
$$

where in the last expression we have used the 4-momentum conservation relation $p-k_{1}-k_{2}-k_{3}=0$ and we consider the electron a massless particle 
with respect to the $\mu^{+}\left(m_{e} \approx 0.5 \mathrm{MeV}\right)$. Plugging these expressions into (4-13) we obtain

$$
\sum_{\text {spin }}|A|^{2}=31 G_{F}^{2}\left(m_{\mu}^{3} E_{\nu_{e}}-2 \mu^{2} E_{\nu_{e}}^{2}\right) .
$$

The physical important aspect is that the square module of the amplitude is a function depending only on $E_{\nu_{e}}$ so is independent of $E_{e^{+}}$and $E_{\bar{\nu}_{\mu}}$. When one calculates the total decay rate, this mathematical characteristic implies a really interesting and non intuitive physical consequence: the energy spectrum of the positron is the same as the $\bar{\nu}_{\mu}$ spectra. In fact, if we put all together and perform the integral respect to $E_{\nu_{e}}$ in the range stated by the kinematics $m_{\mu} / 2-E_{e^{+}}<E_{\nu_{e}}<m_{\mu} / 2$ we obtain

$$
\frac{\mathrm{d} \Gamma}{\mathrm{d} E_{e^{+}\left(\bar{\nu}_{\mu}\right)}}=\frac{G_{F}^{2} m_{\mu}^{2}}{4 \pi^{3}} E_{e^{+}\left(\bar{\nu}_{\mu}\right)}\left(1-\frac{4 E_{e^{+}\left(\bar{\nu}_{\mu}\right)}}{3 m_{\mu}}\right) .
$$

The shape of this distribution is shown in Fig.4.3 as the solid line.

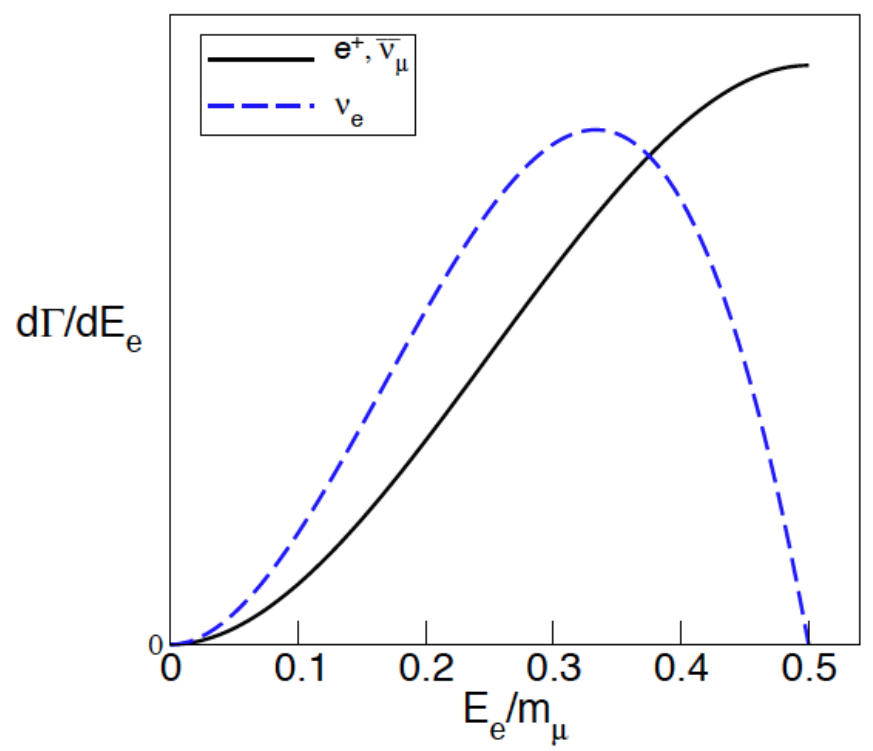

Figure 4.3: The differential decay rate of the $\mu^{+}$decay at rest with respect the $E_{\nu_{e}} / m_{\mu}$ (dashed) and $E_{\bar{\nu}_{\mu}\left(e^{+}\right)} / m_{\mu}$ (solid).

The differential decay rate shape function is telling us that the most probable energy for the emitted positron $\left(\right.$ or $\left.\bar{\nu}_{\mu}\right)$ is when $E_{e^{+}}=m_{\mu} / 2$. This case also corresponds to the case when we have the emission of the positron on the same line of sight as $\bar{\nu}_{\mu}$ and $\nu_{e}$ but in the opposite direction. This can be explained as well following the helicities of the initial and final state. Because we have three massless particles in the final state, their helicities are fixed: $e^{+}$is right-handed, $\nu_{e}$ left-handed and $\bar{\nu}_{\mu}$ right-handed. The total final spin must be equal to the initial one that is $1 / 2$ in our case. So as we see in Fig.4.4 neutrinos will be emitted in the opposite direction respect to the positron. 
Said this, if the $\mu^{+}$is assumed to be at rest, there is no preferred direction of emission for the positron. That is not true when $\mu^{+}$has an initial momentum different from zero, the angular momentum conservation favours the emission of the positron backwards with respect to the direction of $p_{\mu}$. Another way to see this is looking at the square module of the amplitude. The most probable event is the one that maximize $|A|^{2}$ that is the product of two scalar products (in the 4-dimensional space) so that in the general case

$$
\sum_{\text {spin }}|A|^{2} \propto\left(E_{\mu} E_{\nu_{e}}-\vec{p}_{\mu} \cdot \vec{E}_{\nu_{e}}\right)\left(E_{e^{+}} E_{\bar{\nu}_{\mu}}-\vec{E}_{e^{+}} \cdot \vec{E}_{\bar{\nu}_{\mu}}\right) .
$$

Due to the angular momentum conservation $\vec{p}_{\mu} \cdot \vec{E}_{\nu_{e}} \geq 0$ and $\vec{E}_{e^{+}} \cdot \vec{E}_{\bar{\nu}_{\mu}} \leq 0$ are more probable. So joining these conditions we have that the most probable configuration will be the one where the emission of $\nu_{e}$ is orthogonal to $p_{\mu}$ direction and $\vec{E}_{e^{+}} \cdot \vec{E}_{\bar{\nu}_{\mu}}=-1$.

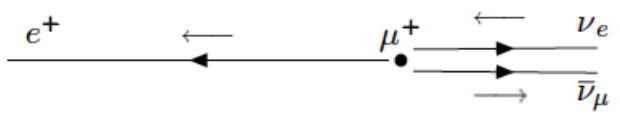

Figure 4.4: Representation of the final state helicities.

Let us say something about the isotropy we mentioned in the introduction. Since $\pi^{+}$spin is 0 , the directions of the decay products are isotropically selected once it is considered at rest. Even if the neutrino spectrum is not isotropic with respect to the direction of the $\mu^{+}$emission, in our case the neutrino detection baseline is $16 \mathrm{~km}$ that is 6 order of magnitude bigger than the average muon range to stop at the source $(\sim 1 \mathrm{~cm})$. This implies that the difference of the flux of the opposite direction of the muon decay is of the order of $(1 \mathrm{~cm} / 16 \mathrm{~km})^{2} \approx 10^{-12}$, that is clearly negligible. Therefore, we can safely regard neutrino emission coming from $\pi^{+}$decay at rest as isotropic.

To conclude this section we summarize in Fig.4.5 the two different unoscillated energy spectrum of the neutrinos emitted. 


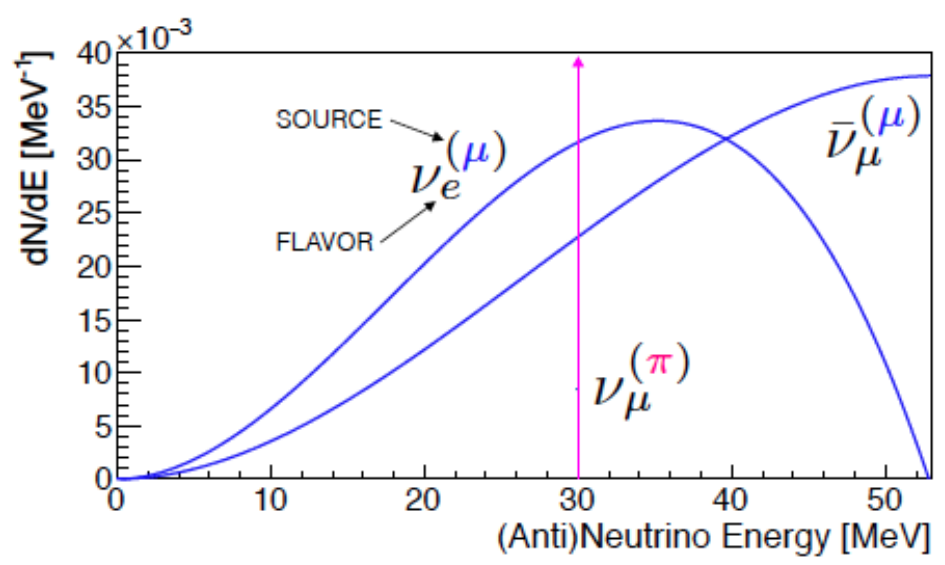

Figure 4.5: Unoscillated spectrum of emitted neutrinos fro $\pi^{+}$DAR.

\section{2}

\section{Neutrino Oscillation Probability $P\left(\nu_{\mu} \rightarrow \nu_{e}\right)$}

The neutrino probability transition $\nu_{\mu} \rightarrow \nu_{e}$ using the standard PMNS parametrization $(2-47)$ is

$$
\begin{aligned}
P_{\nu_{\mu} \rightarrow \nu_{e}}= & +s_{23}^{2} S_{13}^{2} \sin ^{2} \Delta_{31} \\
& -\sin \delta c_{13} S_{12} S_{13} S_{23} \sin \Delta_{31} \sin \Delta_{21} \sin \left(\Delta_{31}-\Delta_{21}\right) \\
& +\cos \delta c_{13} S_{12} S_{23} S_{13} \sin \left(\Delta_{31}-\Delta_{21}\right) \cos \Delta_{31} \sin \Delta_{21} \\
& +4 s_{12}^{2} c_{13}^{2} s_{23}^{2}\left(c_{12}^{2}+s_{13}^{2} s_{12}^{2}\right) \sin ^{2} \Delta_{21} \\
& -2 S_{13}^{2} s_{23}^{2} s_{12}^{2} \cos \left(\Delta_{31}-\Delta_{21}\right) \sin \Delta_{31} \sin \Delta_{21}
\end{aligned}
$$

where $s_{i j}=\sin \theta_{i j}, S_{i j}=\sin 2 \theta_{i j}$ and $\Delta_{i j}=\Delta m_{i j}^{2} L / 4 E$. The second and the third terms are the ones that involve the $\delta$ phase: the second is the one responsible for the $\mathrm{CP}$ violation and the third is the one that is $\mathrm{CP}$ conserving. For the anti-neutrino channel, the sign of CP phase must be flipped. Our experiment maximizes the $\sin \delta$ term at first approximation indeed. In our case, the energy spectrum of $\nu_{\mu}$ coming from $\pi^{+}$is a tiny dispersion around the value $30 \mathrm{MeV}$. At this energy the ideal baseline to be used should give $\Delta_{31}=\pi / 2$

$$
\Delta_{31}=\frac{\Delta m_{31}^{2} L}{4 E}=\frac{\pi}{2} \rightarrow L \approx 15 \mathrm{~km} .
$$

The baseline we will choose is slightly higher than this $(16 \mathrm{~km})$ because what the experiment is sensitive to is the difference between neutrino and antineutrino events that is optimal at a slightly higher value. The quantitative evaluation of this consideration will be presented in the next section. The order of the terms in (4-20) is not casual. At our baseline and for energies of tens of $\mathrm{MeV}$ the first term is the leading order term $\approx 10^{-1}$, the second term $\approx 10^{-2}$, the third $\approx 10^{-3}$ and the last two respectively $\approx 10^{-4}, \approx 10^{-5}$. 
The other important physical aspect is that the term that contains $\sin \delta$ is proportional to all the other leptonic mixing angles and their uncertainties will reduce our sensitivity of measuring $\delta$. In Fig. 4.6 we show how the value of $P\left(\nu_{\mu} \rightarrow \nu_{e}\right)$ varies with respect to the baseline at a fixed energy $E=30 \mathrm{MeV}$ to show that around $15 \mathrm{~km}$ one has the highest values for different possible values of $\delta$.

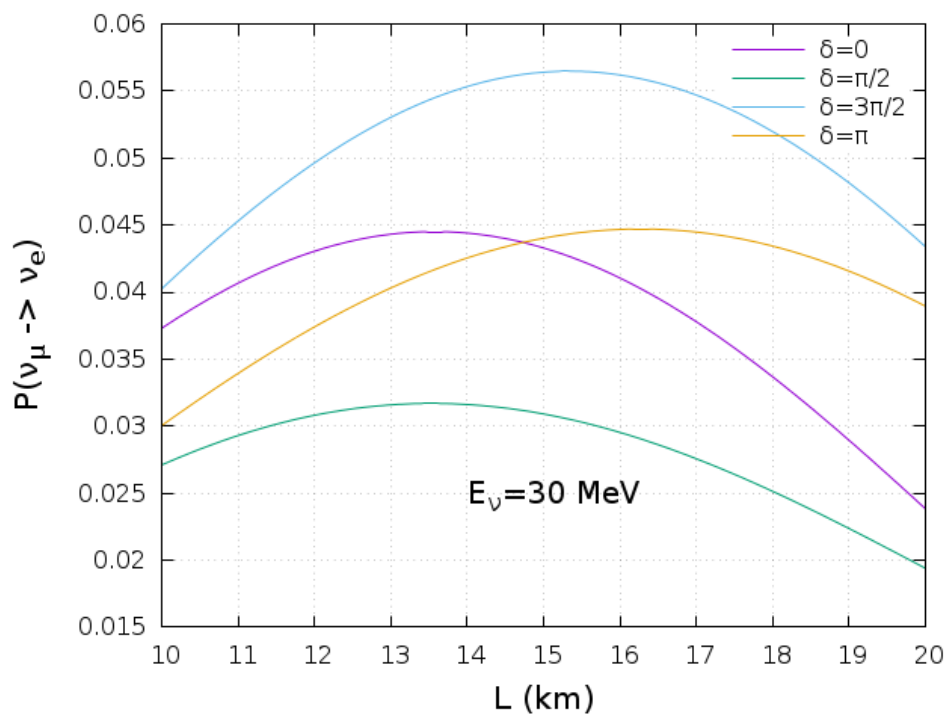

Figure 4.6: $P\left(\nu_{\mu} \rightarrow \nu_{e}\right)$ as a function of the baseline $L$ at a fixed energy $E=30 \mathrm{MeV}$ for different values of $\delta=0, \pi / 2, \pi 3 / 2$ and $\pi$. 
Chapter 4. A Novel Method to Measure the Leptonic CP-asymmetry:

Experimental Set Up

\section{3}

\section{Events Number for Neutrinos and Anti-Neutrinos}

To quantify the sensitivity to explore $\mathrm{CP}$ violation one has to compute the number of events. The detector we have in mind would be a big tank filled with liquid scintillator, a target material commonly use in neutrino experiments. This material is rich in "free" proton. The word "free" has to be intended in the strong interaction sense. So the interaction we will consider for $\bar{\nu}_{e}$ is the IBD on free protons whose approximated cross section is given in (2-45)

$$
\bar{\nu}_{e}+p \rightarrow n+e^{+}
$$

For the $\bar{\nu}_{e}$ number of events we use the formula

$$
\frac{\mathrm{d} N_{\bar{\nu}_{e}}}{\mathrm{~d} E_{\bar{\nu}_{e}}}\left(E_{\bar{\nu}_{e}}\right)=\frac{n f(B) T}{4 \pi L^{2}} \Phi\left(E_{\bar{\nu}_{e}}\right) \cdot P_{\bar{\nu}_{\mu} \rightarrow \bar{\nu}_{e}}\left(E_{\bar{\nu}_{e}}, L, \theta_{23}, \delta\right) \cdot \sigma_{I B D}\left(E_{\bar{\nu}_{e}}\right)
$$

where $\Phi\left(E_{\bar{\nu}_{e}}\right)$ is a shape function normalized to unity that takes into account the $\bar{\nu}_{\mu}$ energy production distribution. In the oscillation probability function we will let vary just a mixing parameters, i.e. $\theta_{23}$, except for $\delta$ that is what we want to measure. The choice of letting specifically $\theta_{23}$ varying will be explained in details in the next chapter where we look at effects that this variation has on the sensitivity of $\delta$ measurement. We summarize all the constants in

\begin{tabular}{|c|c|c|}
\hline $\mathrm{n}$ & (proton-fraction) $(M) N_{A}=(0.1) \frac{1}{\mathrm{~g}}(100)$ kton $N_{A}$ & $\begin{array}{l}\text { We assume a proton mass } \\
\text { fraction of } 10 \% \text { (water like } \\
\text { mass fraction) and a size of } \\
100 \text { kton that gives the nu- } \\
\text { merical value of } n=6.02 \times \\
10^{33}\end{array}$ \\
\hline $\mathrm{T}$ & exposure: 5 years & $\begin{array}{l}\text { We are assuming a } 5 \text { years } \\
\text { exposure taking into con- } \\
\text { sideration the calculations } \\
\text { made by [33] }\end{array}$ \\
\hline $\mathrm{f}(\mathrm{B})$ & {$[B] \times 10^{22} \frac{\# \nu}{\text { MWyear }}$} & $\begin{array}{l}\text { Here as well we inspired to } \\
{[33] \text { to have a total num- }} \\
\text { ber of events of the order of } \\
1200 \text { (each type of neutrino) } \\
\text { for a } \mathrm{B}=36 \text {. The } B \text { depen- } \\
\text { dency of the flux normaliza- } \\
\text { tion is a sort of classification } \\
\text { for the different realizable } \\
\text { configurations } B \in[1,100]\end{array}$ \\
\hline
\end{tabular}
Table.4.1. Here we introduced a free parameter $B$ that takes into account

Table 4.1: $\mathrm{d} N_{\bar{\nu}_{e}} / \mathrm{d} E_{\bar{\nu}_{e}}$ parameters.

the possible experimental configuration, i.e. variation on the exposure time, 
different beam power, different detector size, etc. . In Fig.4.7 we plot the $\bar{\nu}$ number of events for different values of $\delta$ and for a fixed $B=30$. We choose this factor $B=30$ because it represents the effective limit that should be reached by the experimental realization. This factor corresponds to a $T=10$ years exposure time, $5 M W$ accelerator power and 300 kton fiducial mass.

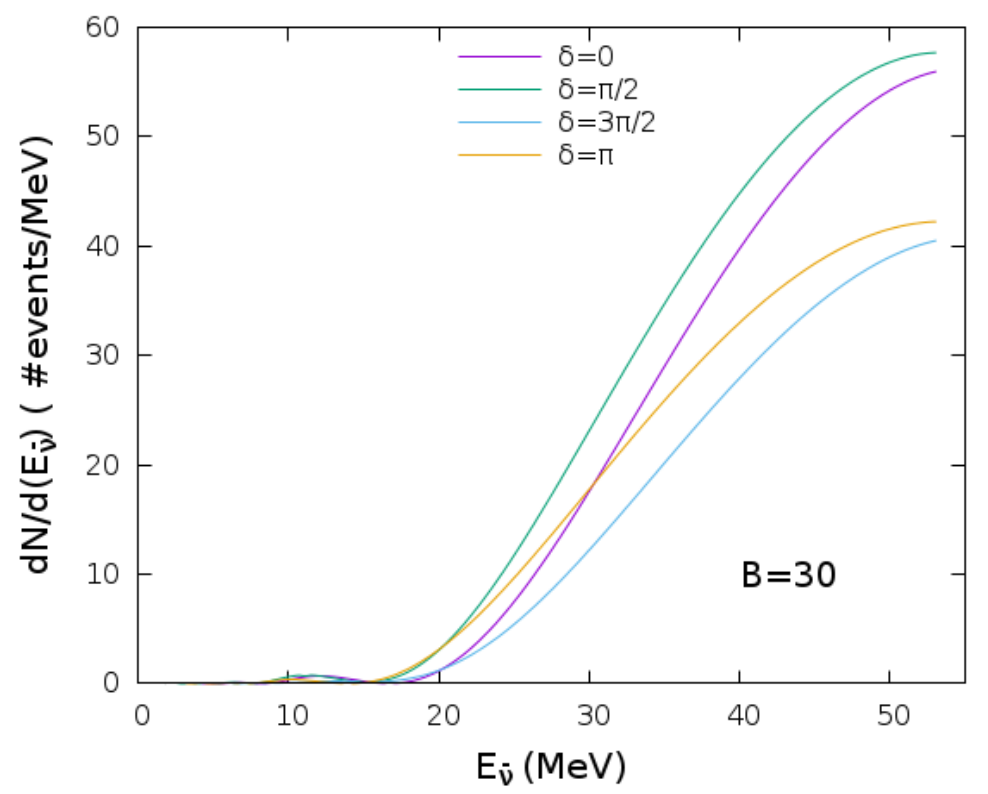

Figure 4.7: $\mathrm{d} N_{\bar{\nu}_{e}} / \mathrm{d} E_{\bar{\nu}_{e}}$ as a function of neutrino energy $E_{\bar{\nu}_{e}}$ for a fixed $B=30$ factor at different values of $\delta=0, \pi / 2, \pi 3 / 2$ and $\pi$.

The case of $\nu_{e}$ detection is more complicated from the interaction point of view. The process one should look at to detect $\nu_{e}$ is

$$
\nu_{e}+n \rightarrow p+e^{-}
$$

Obviously neutrons are only present inside nuclei, so the realistic interaction one must consider is

$$
\nu_{e}+{ }_{n}^{m} \mathrm{X} \rightarrow e^{-}+{ }_{n+1}^{m} \mathrm{X}^{+}+\gamma^{\prime} s
$$

Neutrino interactions on nuclei in the tens-of-MeV range are relatively poorly understood theoretically, in terms of both the interaction rate and the angular energy distributions of the resulting interaction products. This happen because at that range of energy one must consider the whole nucleus involved in the interaction, taking into account all its nuclear internal energy states. The most recent numerical estimations are from Scholberg and Formaggio et al. [34, 35]. In Fig.4.8 we have a plot from Scholberg that shows all the theoretical cross section estimations. We are actually interested just in one value at a specific energy i.e. $30 \mathrm{MeV}$. Looking at the plot it is evident that 
$\nu_{e}+{ }_{82}^{208} \mathrm{~Pb} \rightarrow e^{-}+{ }_{83}^{208} \mathrm{Bi}^{+}$is the interaction with the highest value $(\sim 50$ times bigger than IBD) that has a final state detectable with high efficiency by our detector.

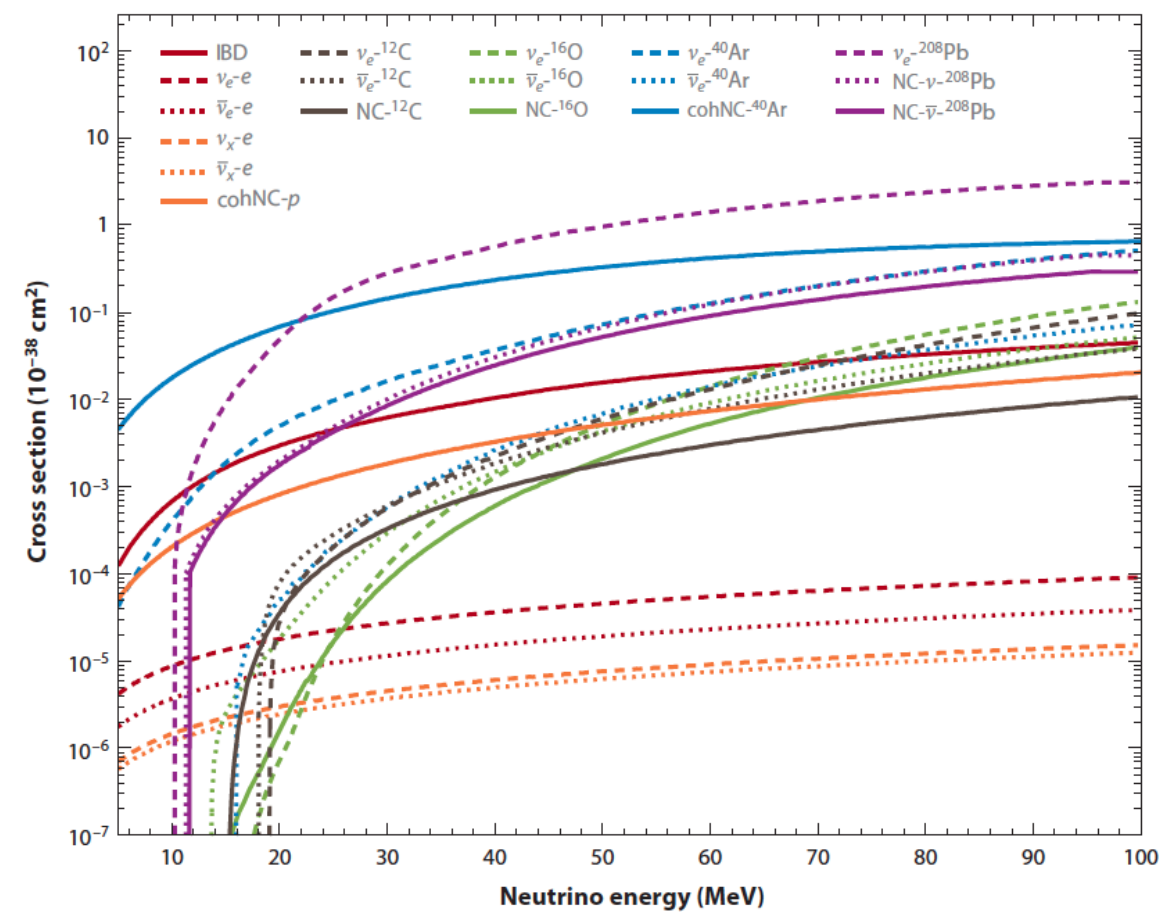

Figure 4.8: Numerical estimation of $\nu_{e}$ and $\bar{\nu}_{e}$ relevant interaction cross sections for different targets. Abbreviations: IBD, inverse beta decay; NC, neutral current.

The experiment proposes to use neutron rich stable elements (lead for example) as target to detect neutrinos because of their high cross section. The idea is to highly dope the liquid scintillator with this material to increase the number of $\nu_{e}$ events. Even if this doping could affect the transparency of the scintillator it would not interfere with the detection. Indeed, the detection process is actually based on a opaque scintillator where the scintillation light will not be collected at the edges of the detector by photosensors. Rather, it gets collected close to the interaction vertex by a dense lattice of optical fibers crossing the whole detector. In Fig.4.9 a $e^{-}$and a $e^{+}$detection simulation is shown. Each pixel in the picture represent a fiber and the color indicates the number of photons collected by it. The two events have really characteristic signatures: the $e^{-}$(left) has track-like energy deposition resulting from the particles' ionization; for the case of $e^{+}$there is also a multiple energy depositions due to Compton scattering of the positron's annihilation $\gamma$ 's.

The two biggest unknown in the $\nu_{e}$ detection are the cross section and the heavy material loading of the scintillator. At a phenomenological level these uncertainties can be taken into account introducing the parameter $\eta$ 


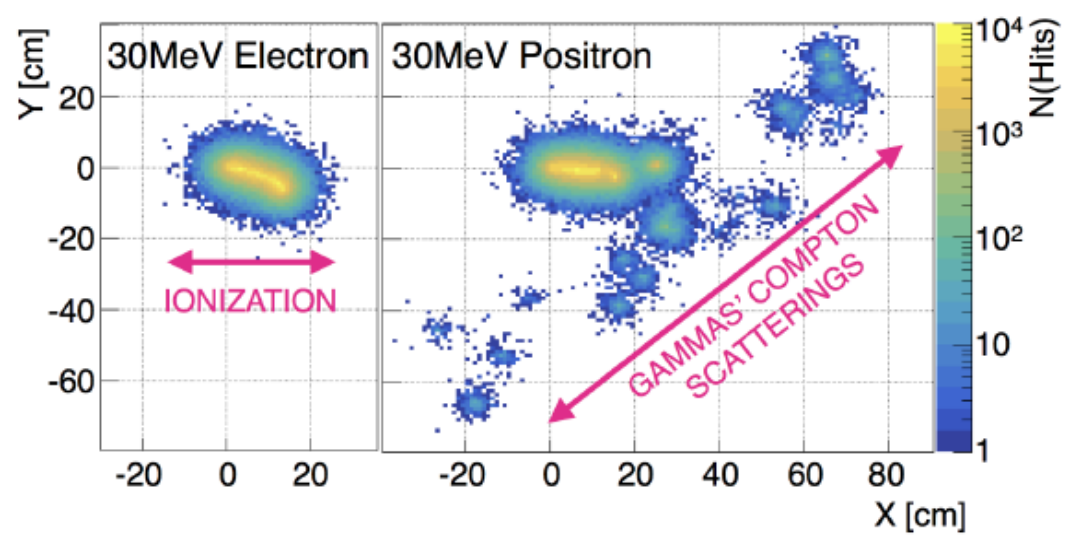

Figure 4.9: Simulation of a $e^{-}$(left), $e^{+}$(right) detection. Each pixel represents an optical fiber and the colour indicates the number of photons collected by it. Appear evident the different signature that will help in the events discrimination.

$$
N_{\nu_{e}}\left(\hat{\theta}_{23}, \delta=0\right)=\eta \cdot N_{\bar{\nu}_{e}}\left(\hat{\theta}_{23}, \delta=0\right) \text {. }
$$

This parameter express the ratio between the number of events of $\bar{\nu}_{e}$ over $\nu_{e}$ for the case of no $\mathrm{CP}$ violation and the same values of the other mixing parameters. 


\section{4}

\section{The Physical Background}

In this section we briefly introduce how we take into account our main background for $\nu_{e}$ and $\bar{\nu}_{e}$ signals. We use the word main to stress the fact that in this section we will only address the physical intrinsic background that does not depend on the specific experimental set up.

As we have seen in the first section of this chapter, our neutrinos are coming from DAR $\pi^{+}$decay produced by the collision of accelerated proton on carbon or mercury target. In this process $\pi^{-}$are also produced at almost the same rate $\left(\pi^{-} / \pi^{+}=0.62[36]\right)$ and they also decay following the chain

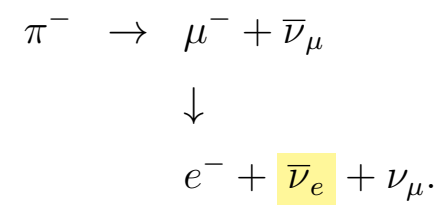

The highlighted $\bar{\nu}_{e}$ that survive the oscillation will mimic exactly our $\bar{\nu}_{e}$ signal coming from $\pi^{+}$DAR. Fortunately the $\pi^{-}$, and the successive produced $\mu^{-}$, are absorbed at the target location by forming $\pi^{-}$-mesic and $\mu^{-}$-mesic atoms respectively. From FLUKA hadron simulation package [36] the survival ratio for antineutrinos is $\bar{\nu}_{e} / \pi^{-}=1.9 \cdot 10^{-3}$.

Taking into account this simulation results we defined the background spectrum as

$$
\frac{\mathrm{d} N_{\bar{\nu}_{e}}^{b k g}}{\mathrm{~d} E_{\bar{\nu}_{e}}}\left(E_{\bar{\nu}_{e}}\right)=C \cdot \frac{n f(B) T}{4 \pi L^{2}} \Phi^{\mu^{-}}\left(E_{\bar{\nu}_{e}}\right) \cdot P_{\bar{\nu}_{e} \rightarrow \bar{\nu}_{e}}\left(E_{\bar{\nu}_{e}}, L, s_{23}^{2}{ }^{\text {true }}, \delta\right) \cdot \sigma_{I B D}\left(E_{\bar{\nu}_{e}}\right),
$$

where $C=\pi^{-} / \pi^{+} \times \bar{\nu}_{e} / \pi^{-}=1.18 \cdot 10^{-3}[36], \Phi^{\mu^{-}}\left(E_{\bar{\nu}_{e}}\right)$ is the shape function of the $\nu_{e}$ for the case of $\mu^{+}$decay, $\delta=0$ and $s_{23}^{2}{ }^{\text {true }}$ can be $[L O W, M I D, H I G H]$. In Fig.4.10 We show the $\bar{\nu}_{e}$ spectrum without the background (black) and the background (red) from eq.(4-27) for the reference values $\delta=0, \eta=0.3$ and $s_{23}^{2}=0.5$.

The other main background to the $\nu_{e}$ signal comes from the same $\pi^{+}$ DAR chain

$$
\begin{aligned}
\pi^{+} \rightarrow & \mu^{+}+\nu_{\mu} \\
& \downarrow \\
& e^{+}+\nu_{e}+\bar{\nu}_{\mu} .
\end{aligned}
$$

In this case the highlighted $\nu_{e}$ at $30 \mathrm{MeV}$ that will survive the oscillation 
Chapter 4. A Novel Method to Measure the Leptonic CP-asymmetry:

Experimental Set Up

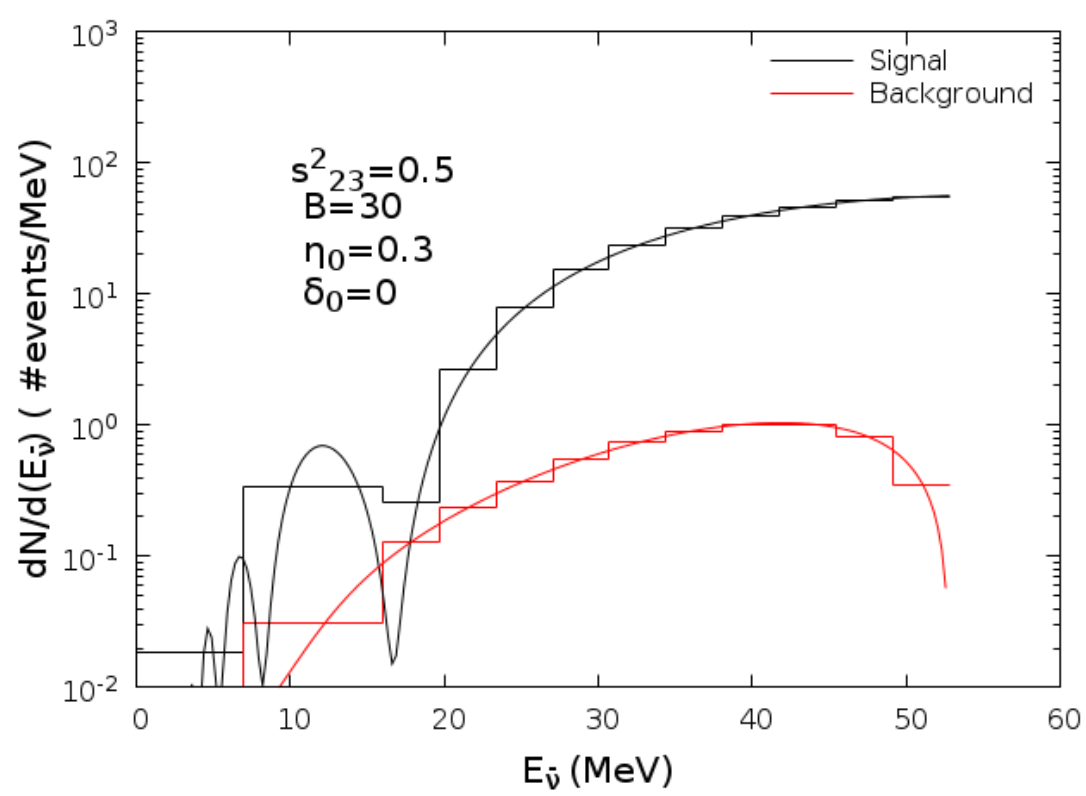

Figure 4.10: $\mathrm{d} N_{\bar{\nu}_{e}} / \mathrm{d} E_{\bar{\nu}_{e}}$ (black) and its background (red).

will be indistinguishable from the $\nu_{e}$ coming from the $\nu_{\mu}$ oscillation. In this case we choose a conservative approach considering $N_{\nu_{e}}^{b k g}=N_{\nu_{e}}$. 


\section{5}

\section{Sensitivity Study: $\chi^{2}$ Definition}

In order to quantify the sensitivity of our experiment to established the $\mathrm{CP}$ violation, if $\sin \delta$ is different from zero, one has to define a $\chi^{2}$ function. In our case we use a convention that is commonly used by other experiments $[16,23]$ i.e. we assign a zero value to the $\chi^{2}$ for $\delta=0, \pi$. In this scenario our $\chi^{2}$ is

$$
\chi^{2}\left(\delta_{\text {true }}\right)={ }_{\min }\left\{\left.\left[\chi_{\nu_{e}}^{2}\left(\delta_{\text {true }}\right)+\chi_{\bar{\nu}_{e}}^{2}\left(\delta_{\text {true }}\right)\right]\right|_{\delta=[0, \pi]}\right\}
$$

where we defined $\chi_{\nu_{e}}^{2}\left(\delta_{\text {true }}\right)$ and a $\chi_{\bar{\nu}_{e}}^{2}\left(\delta_{\text {true }}\right)$ as

$$
\chi_{\nu_{e}}^{2}\left(\delta_{\text {true }}\right)={ }_{\min \left[s_{23 f i t}^{2}, \eta_{f i t}\right]}\left\{\frac{\left(N_{\nu_{e}}^{\text {true }}\left(\delta_{\text {true }}, s_{23 \text { true }}^{2}, \eta_{\text {true }}\right)-N_{\nu_{e}}^{f i t}\left(\delta=[0, \pi], s_{23 f i t}^{2}, \eta_{f i t}\right)\right)^{2}}{\sigma_{\nu_{e}}^{2}}\right\}
$$

$$
\chi_{\bar{\nu}_{e}}^{2}\left(\delta_{\text {true }}\right)={ }_{\min \left[s_{23 f i t}^{2}\right]}^{2}\left\{\sum_{i} \frac{\left(N_{\bar{\nu}_{e}}^{\text {true }}\left(\delta_{\text {true }}, s_{23 t r u e}^{2}\right)-N_{\bar{\nu}_{e} i}^{f i t}\left(\delta=[0, \pi], s_{23 f i t}^{2}\right)\right)^{2}}{\sigma_{\bar{\nu}_{e}}^{2}}\right\},
$$

where the minimization with respect to $s_{23 \text { fit }}^{2}$ and $\eta_{f i t}$ are computed for the two terms simultaneously.

We have considered the variances $\sigma_{\nu_{e}}$ and $\sigma_{\bar{\nu}_{e}}$ as statistical based only so that $\sigma_{\nu_{e}}^{2}=N_{\nu_{e}}^{\text {true }}, \sigma_{\bar{\nu}_{e}}^{2}=N_{\bar{\nu}_{e}}^{\text {true }}$. For the anti-neutrino we choose not to use just the total number of events. With the introduction of an energy binning it is possible to take into account not just the variations on the total number of events but also the spectral distortions. The binning was performed observing Fig.4.7. As one can see the vast majority of the events are distributed above $\sim 16 \mathrm{MeV}$ (99.6\% of total events number). For this reason we choose to divide the $[1.83,16] \mathrm{MeV}$ region in just two bins. One $[1.83,7.00] \mathrm{MeV}$ and the other $[7.00,16.00] \mathrm{MeV}$. This choice took into account the presence of the tiny second oscillation maxima that one can see has its peak at $\sim 12 \mathrm{MeV}$. The $[16.0,52.83] \mathrm{MeV}$ region was divided in 10 equally spaced bins.

Both $\chi_{\bar{\nu}_{e}}^{2}$ and $\chi_{\nu_{e}}^{2}$ are minimized with respect to $s_{23}^{2}$ and $\eta$ (just for $\nu_{e}$ case) to take into account the effect that their uncertainties have on $\delta$ sensitivity. To take into account also the systematic errors on these two parameters we added the respective pull terms defined as 


$$
\chi_{\text {pull }}^{2}={ }_{\min \left[s_{23 f i t}^{2}\right]}\left\{\frac{\left(s_{23}^{2}-\left(s_{23}^{\mathrm{ref}}\right)^{2}\right)^{2}}{\sigma_{s_{23}^{2}}^{2}}+\frac{\left(\eta-\eta_{\mathrm{ref}}\right)^{2}}{\sigma_{\eta}^{2}}\right\}
$$

where $\sigma_{\eta}=10 \% \eta_{\text {ref. }}$ For $\sigma_{s_{23}^{2}}^{2}$ and $s_{23}^{2}$ we will use the three cases showed in 3: the low case $s_{23}^{2}=0.441 \pm 0.025$, the mid case $s_{23}^{2}=0.5 \pm 0.042^{1}$ and the high case $s_{23}^{2}=0.587 \pm 0.022$. In this chapter we will use the mid test value otherwise it will be mentioned.

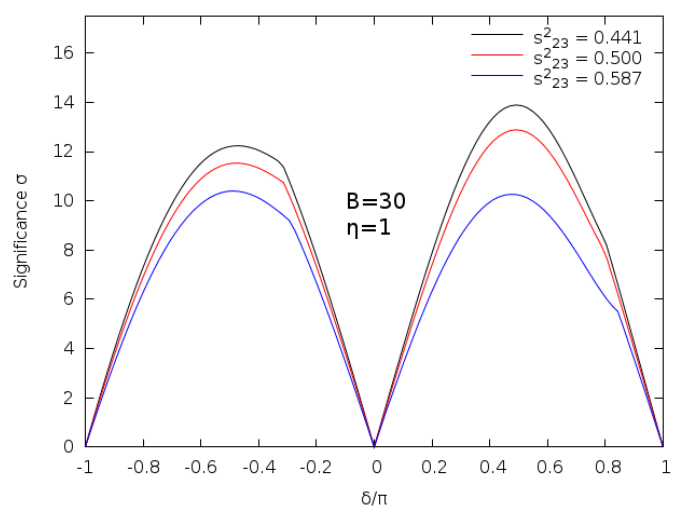

Figure 5.1: Sensitivity plot in number of sigma respect to the true value of $\delta$. We compared the three $s_{23}^{2}$ cases [LOW,MID,HIGH] without marginalization of $s_{23}^{2}$ and $\eta$ just to appreciate the overall difference between them.

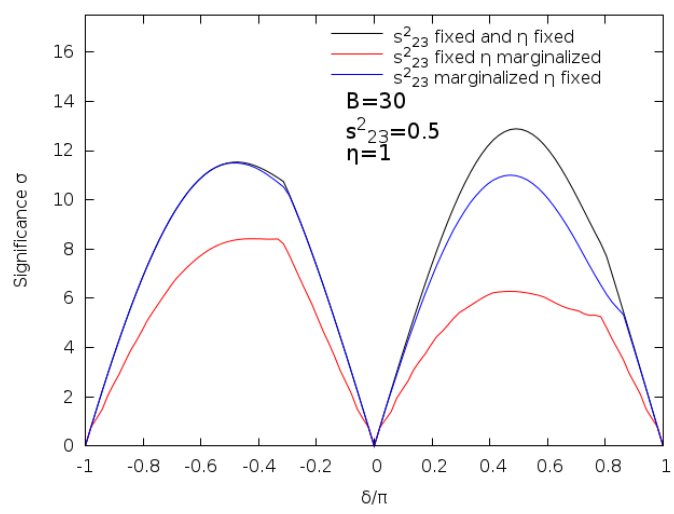

Figure 5.2: Sensitivity plot in number of sigma respect to the true value of $\delta$. We compared the shapes for the absence of marginalization (black), the marginalization of just $s_{23}^{2}$ and the marginalization of just $\eta$ parameter.

To better understand the effects of this minimization it is instructive to see the numerical values of the number of events

$N_{\nu_{e}}=B \eta(-10.453 \sin \delta-0.169 \cos \delta+33.839) \approx B \eta(-10.453 \sin \delta+33.839)$

$N_{\bar{\nu}_{e}}=B(7.317 \sin \delta+2.462 \cos \delta+31.210)$,

${ }^{1}$ Due to the fact that 0.5 is just a test value (no experiment has favoured it but it is not excluded yet) the error we choose is just the difference between the high best fit value with 0.5 divided by two $((0.587-0.5) / 2 \sim 0.042)$. 
where all the numerical factor are calculated using the parameters in Table 3.3 except for $s_{23}^{2}=0.5$ as mentioned before.

The most important difference between the two functions is the magnitude of the $\cos \delta$ factor that in the $\nu_{e}$ is practically null. This is the result of the fact that $N_{\nu_{e}} \propto P_{\mu \rightarrow e}(E=30 \mathrm{MeV})$ whereas $N_{\bar{\nu}_{e}}$ is an integral of a convolution of flux $\times \sigma_{I B D} \times P_{\bar{\mu} \rightarrow \bar{e}\left(E_{\bar{\nu}}\right)}$ over the energy range $[1.83,52.8]$. In order to see the dependence of $s_{23}^{2}$ in $N_{\nu_{e}}$ and $N_{\bar{\nu}_{e}}$ we can look the probability in (4-20), beyond the factors $\sin \delta$ and $\cos \delta$, this equation also contains the parameter $s_{23}^{2}$ in all the terms. With this in mind and defining $s_{23}^{2}=x$ one obtains

$$
\begin{aligned}
& N_{\nu_{e}}=B \eta(-10.4 \cdot(2 \sqrt{x(1-x)}) \sin \delta+33.8 \cdot(2 x)) \\
& N_{\bar{\nu}_{e}}=B(7.3 \cdot(2 \sqrt{x(1-x)}) \sin \delta+2.5 \cdot(2 \sqrt{x(1-x)}) \cos \delta+31.2 \cdot(2 x)) .
\end{aligned}
$$

Substituting these expressions into the $\chi^{2}$ function in the end one obtains for $B=\eta=1$

$$
\chi_{\nu_{e}}^{2}\left(\delta_{\text {true }}, x\right)=\frac{437.1 x(1-x) \sin ^{2} \delta}{-20.9 \sin \delta \sqrt{x(1-x)}+67.7 x}
$$

$$
\chi_{\bar{\nu}_{e}}^{2}\left(\delta_{\text {true }}, x\right)=\frac{(14.6 \sin \delta \sqrt{x(1-x)}+4.9 \cos \delta \sqrt{x(1-x)} \pm 4.9 \sqrt{x(1-x)})^{2}}{14.6 \sin \delta \sqrt{x(1-x)}+4.9 \cos \delta \sqrt{x(1-x)}+62.4 x}
$$

where the - sign stands for $\delta_{f i t}=0$ and the + for $\delta_{f i t}=\pi$ whereas for the $\nu_{e}$ contribution there is no difference between the two cases.

Now we have the tools to understand how $\eta$ and $x \equiv s_{23}^{2}$ interfere with $\delta$ sensitivity. For the $\eta$ case it's easy to see that it enters in the total $\chi^{2}$ function as a multiplicative factor of the $\nu_{e}$ part, therefore, higher values of $\eta$ implies higher overall sensitivity (5-7). As a consequence of the zero $\cos \delta$ coefficient in $N_{\nu_{e}}$ the $\chi_{\nu_{e}}^{2}$ reduces to a single term when it comes to the minimization with respect $\delta_{f i t}=0, \pi$. For this reason the $\eta$ marginalization acts like an overall reduction of the $\delta$ sensitivity (see Fig.5.2).

The discussion becomes a little bit more complicated for $x$. First of all in this case higher values of $x$ reduces the overall sensitivity whereas lower values of $x$ enhance it. This is mathematically caused by the fact that $\chi_{\nu_{e}}^{2}(x=0.587)<$ $\chi_{\nu_{e}}^{2}(x=0.5)<\chi_{\nu_{e}}^{2}(x=0.441)$ almost for the whole $\delta$ range. This trend for the range of $x$ that we are spanning is caused by the terms, in the probability, that are proportional to $s_{23}^{2}(4-20)$ and that determine the magnitude of the $\chi^{2}$ denominator. Therefore in the low $s_{23}^{2}$ scenario we would have a better overall sensitivity, followed by the mid and then the high cases as it's shown in Fig.5.1. What is peculiar is that the marginalization does not have the same effect for positive and negative values of $\delta$. From Fig.5.2 one can see that the positive $\delta$ 
part is more affected than the negative part.

To understand this effect one has to look carefully to eq.(5-8,5-7). In Fig.5.3 we plotted the two $\chi^{2}$ functions using $s_{23}^{2}=0.7$ (solid) and $s_{23}^{2}=0.4$ (dashed) for $\delta_{f i t}=0, \pi$. Because of the absence of the $\cos \delta$ in the $\nu_{e}$ part we see in the plot just one black line that corresponds to both values of $\delta_{f i t}=0, \pi$. This feature cause the $\chi^{2}$ minimum to be $\chi^{2}=\chi_{\nu_{e}}^{2}+\chi_{\bar{\nu}_{e}}^{2}\left(\delta_{f i t}=\pi\right)$ for negative values of $\delta$, otherwise for positive values we have $\chi^{2}=\chi_{\nu_{e}}^{2}+\chi_{\bar{\nu}_{e}}^{2}\left(\delta_{f i t}=0\right)$. We can also see that the $\chi_{\nu_{e}}^{2}$ is more sensible to the $x$ variation for positive values of $\delta$ because in the denominator we have a subtraction of the term proportional to $\sin \delta$ to the constant term as we see in (5-6). Indeed, in Fig. 5.3 the dashed line reaches higher values for $\delta>0$ than $\delta<0$. The $\bar{\nu}_{e}$ part mirroring this feature for $\delta<0$ because of the opposite sign of the $\sin \delta$ term and for $\delta_{f i t}=0$. However this function does not appear into the minimized total $\chi^{2}$ as we stated before, so the marginalization over $x$ has less impact for $\delta<0$.

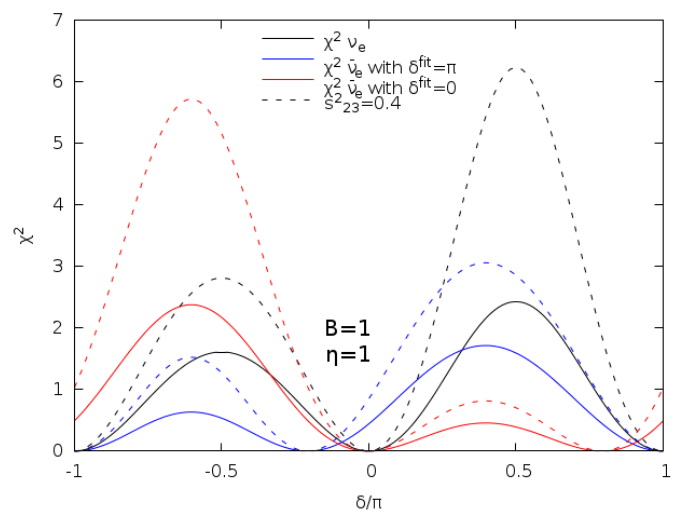

Figure 5.3: Plot of $\chi_{\nu_{e}}^{2}$ and $\left.\chi_{\bar{\nu}_{e}}^{2}\right|_{\delta^{f i t}=0, \pi}$ at fixed $s_{23}^{2}=0.7$ (solid) and $s_{23}^{2}=0.4$ (dashed).

To conclude the section we will present the calculation that motivated our baseline choice of the $16 \mathrm{~km}$. In Fig. 5.4 we show the sensitivity plot at a fixed $\eta$ for $s_{23}^{2}=0.5$ at different baselines. It's easy to see how the $16 \mathrm{~km}$ baseline stands on top of all the others. This trend is independent of the $s_{23}^{2}$ value.

\section{1}

\section{Background Effects on Sensitivity}

Now it is interesting to see the effect of the physical backgrounds shown in the previous chapter on our $\delta$ sensitivity. First of all, due to the fact that both background are coming from survival probabilities, they will be independent from the $\delta$ value. In fact, in our $\chi^{2}$ function, they will appear just in the $\sigma$ 's definition 


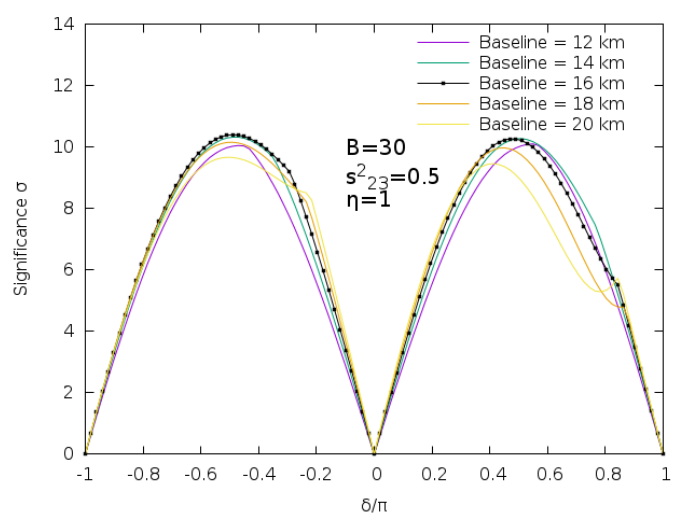

Figure 5.4: Sensitivity plot in number of sigma respect to the true value of $\delta$ at different baselines at a fixed values of $\eta$.

$$
\begin{gathered}
\chi_{\nu_{e}}^{b k g^{2}}\left(\delta_{\text {true }}\right)={ }_{\min \left[s_{23 f i t}^{2}, \eta_{f i t}\right]}\left\{\frac{\left(N_{\nu_{e}}^{\text {true }}+N_{\nu_{e}}^{b k g}-N_{\nu_{e}}^{f i t}-N_{\nu_{e}}^{b k g}\right)^{2}}{\sigma_{\nu_{e}}^{b k g^{2}}}\right\} \\
\chi_{\bar{\nu}_{e}}^{b k g^{2}}\left(\delta_{\text {true }}\right)={ }_{\min \left[s_{23 f i t}^{2}\right]}^{2}\left\{\sum_{i} \frac{\left(N_{\bar{\nu}_{e}}^{t r u e^{i}}+N_{\bar{\nu}_{e}}^{b k g^{i}}-N_{\bar{\nu}_{e}}^{f i t^{i}}-N_{\bar{\nu}_{e}}^{b k g^{i}}\right)^{2}}{\sigma_{\bar{\nu}_{e} i}^{b k g^{2}}}\right\},
\end{gathered}
$$

where

$$
\begin{gathered}
\sigma_{\bar{\nu}_{e} i}^{b k g^{2}}=N_{\bar{\nu}_{e}}^{t r u e}+N_{\bar{\nu}_{e}}^{b k g i} \\
\sigma_{\nu_{e}}^{b k g^{2}}=N_{\nu_{e}}^{t r u e}+N_{\nu_{e}}^{b k g} \\
N_{\bar{\nu}_{e}}^{b k g^{i}}=\int_{E_{i}}^{E_{i+1}} \frac{\mathrm{d} N_{\bar{\nu}_{e}}^{b k g}}{\mathrm{~d} E_{\bar{\nu}_{e}}} \mathrm{~d} E_{\bar{\nu}_{e}} .
\end{gathered}
$$

In Fig.5.5 we show how the presence of the background affects the $\delta$ sensitivity for the $[L O W, M I D, H I G H]$ cases. As one can see, the background obviously reduces our sensitivity but the reduction is at most $\approx 1 \sigma$ along all the possible $\delta$ true values. 

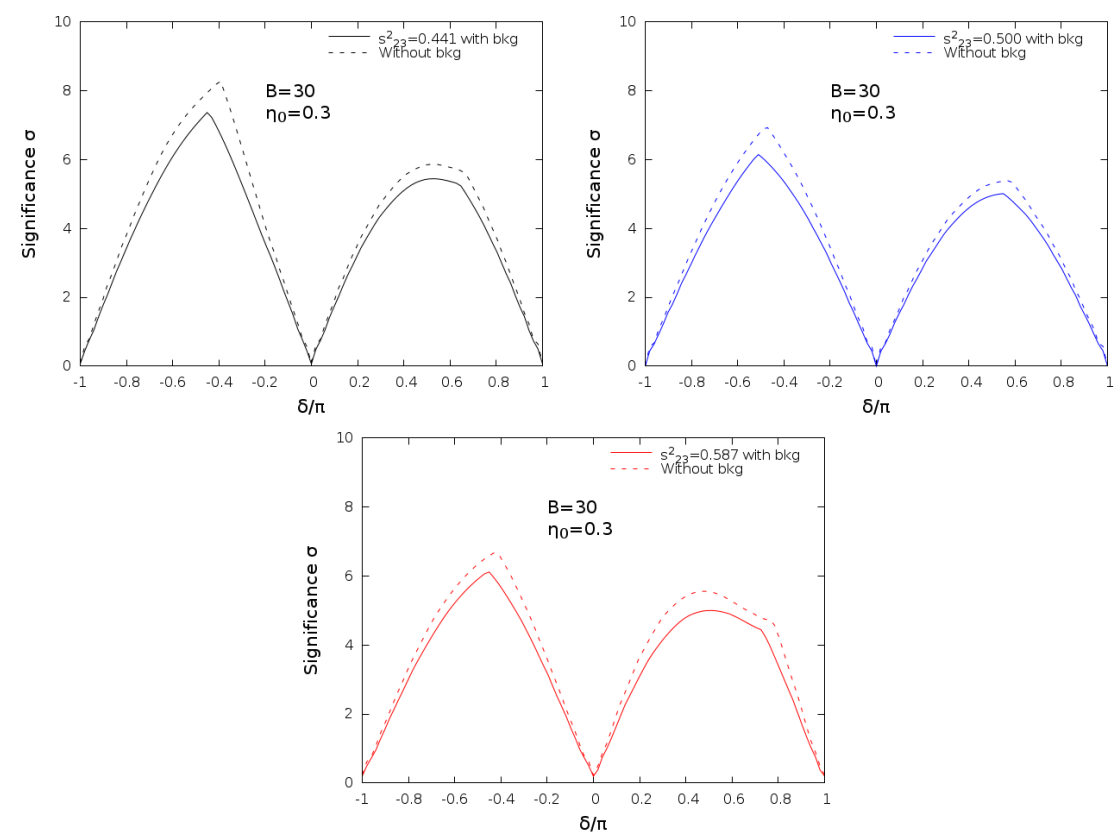

Figure 5.5: Sensitivity plot in number of sigma respect to the true value of $\delta$ for the presence (solid) and the absence (dashed) of background for $s_{23}^{2}=[0.441,0.500,0.587]$.

\section{2}

\section{$\mathrm{B}$ and $\eta$ Scaling}

A very important step to full understand the potential of our experiment is to look at how our sensitivity change respect to parameters $B$ and $\eta$. As we have seen before, $B$ is a parameter that takes into account all the possible experimental configuration taking as basic one

$$
B[1] \Rightarrow 100 \mathrm{kt} \times 5 \text { years } \times 1 \mathrm{MW} \text { beam } \times 10 \% \text { proton fraction } .
$$

We already motivated our choice to take $B=30$ because is reasonably the best experimental configuration with the today technology and it can be represented as

$$
B[30] \Rightarrow 300 \mathrm{kt} \times 10 \text { years } \times 5 \mathrm{MW} \text { beam } \times 10 \% \text { proton fraction } .
$$

In Fig.5.6 we plotted the $\delta$ coverage at $5 \sigma \mathrm{CP}$ violation discovery sensitivity with respect to $B$ for different values of $\eta$, for different $\sigma_{\eta}$ and for $s_{23}^{2}=0.587$. Even in this disfavoured case, our detector has a $35 \%$ coverage for a $\eta=0.3$ and $\sigma_{\eta}=5 \%$ (solid blue) that can become at most $41 \%$ if one can get a better $\sigma_{\eta}$ (dashed blue). For $B$ larger than 30 one can see that the coverage start to saturates with an increment of $26 \%$ over a $B$ increment of 70. From this observation one can deduce that to get a better coverage it is more useful to improve our knowledge of the $\eta$ uncertainty than get a bigger 


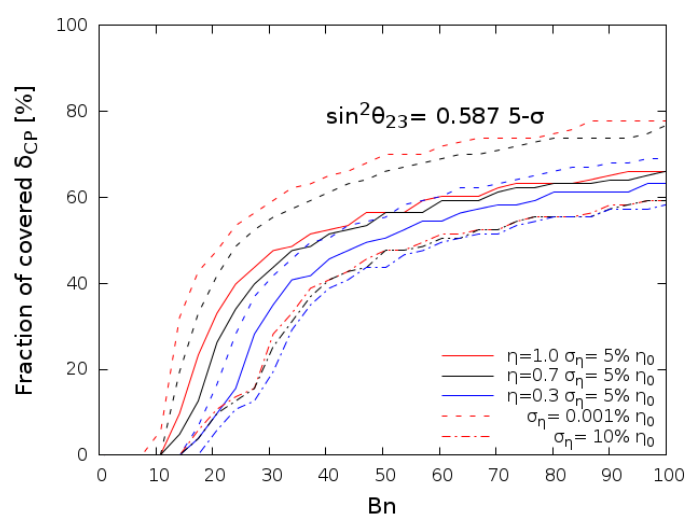

Figure 5.6: Coverage of the range of $\delta$ where the CP violation can be established with a sensitivity equal or bigger than $5 \sigma$ with respect to $B$ parameter. I choose different values of $\eta=0.3$ (blue), 0.7 (black), 1.0 (red) with different uncertainties $\sigma_{\eta}=10 \%$ (dotted dashed), $5 \%$ (solid) and $10^{-4 \%}$ (dashed).

detector. To support this point it is interesting to note that with just a $\eta=0.3$ with a $\sigma_{\eta}=10^{-4 \%}$ one can reach the same coverage (a bigger coverage for $B$ larger than 50) of $\eta=0.7,1.0$. So reducing the $\eta$ uncertainty highly improves our sensitivity and our coverage more than get higher $B$ or $\eta$. To see this effect we show in Fig.5.7 the $\delta$ coverage at $5 \sigma$ with respect to $\eta$ for different values of $\sigma_{\eta}$ and for $B=30$. As it is shown in the figure, for a value of $\eta=0.3$ and for $\sigma_{\eta}=10 \%$ we have a coverage of $\approx 15 \%$ that in the case of $\sigma_{\eta}=5 \%$ for the same $\eta$ increase up to $\approx 35 \%$. However, if we look at the coverage for $\eta=0.7$ for $\sigma_{\eta}=10 \%$ the coverage increases just up to $\approx 24 \%$. This means that we can do good physics just with $\eta=0.3$ and $\sigma_{\eta}=5 \%$. What this value of $\eta$ physically represent is shown in Fig. 5.8. In this plot we shows the value of $\eta$ against the loading portion of $\mathrm{Pb}$ into our liquid scintillator assuming a fixed value of neutrino cross section $\sigma_{\nu_{e}}=50 \times \sigma_{I B D}(E=30 \mathrm{MeV})$. As one can see, the value $\eta=0.3$ correspond to a loading fraction of 0.15 .

For these reasons we have produced all the previous sensitivity plots for the combination $B=30$ and $\eta_{0}=0.3$. This configuration will be the one that we will choose to show our sensitivity final results.

\section{3}

\section{Final Results}

Here we present our final sensitivity results for the combination $B=30$ and $\eta_{0}=0.3$ and $\sigma_{\eta}=5 \% \eta_{0}$. First of all, in Fig. 5.9 we present the final sensitivity plot for the three $s_{23}^{2}$ cases (high,mid and low). This results takes into account the backgrounds we defined in sec.(5.1), the pull terms and the marginalization with respect $\eta$ and $s_{23}^{2}$. As one can see, our setup can exclude at $5 \sigma$ confidence level $\sim 45 \%$ of the $\delta$ interval $[-\pi, \pi]$. 


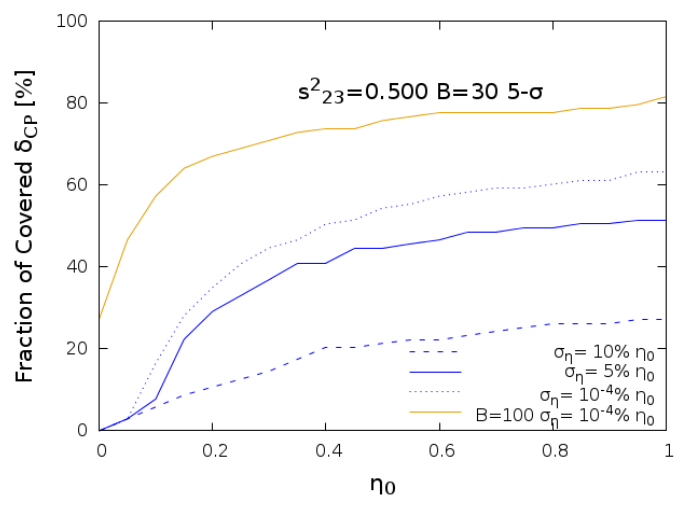

Figure 5.7: Coverage of the range of $\delta$ where the $\mathrm{CP}$ violation can be established with a sensitivity equal or bigger than $5-\sigma$ with respect to $\eta$ parameter for $B=30$ and $s_{23}^{2}=0.500$. We show the results for different $\sigma_{\eta}=10 \%, 5 \%, 10^{-4 \%}$. There is also represented an unreal configuration (solid orange) for $B=100$ and $\sigma_{\text {eta }}=10^{-4 \%}$ that we use as a reference of the best possible coverage.

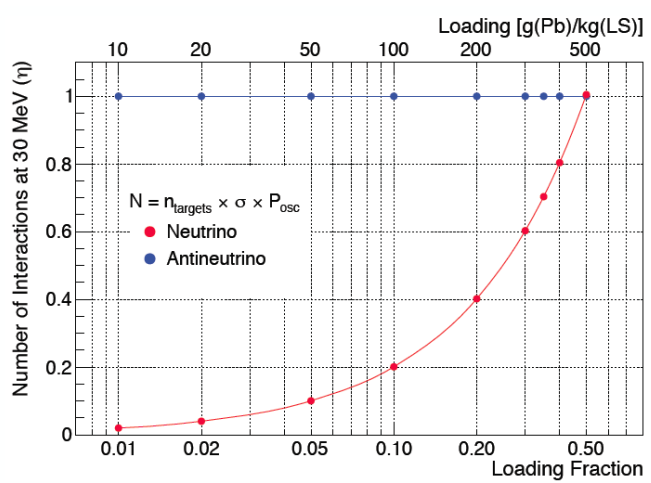

Figure 5.8: This plot shows how to convert the value of our parameter $\eta$ in terms of loading fraction. The number of interaction has been calculated considering a $\sigma_{\nu_{e}}=50 \times \sigma_{I B D}$.

We also show, in Fig. 5.10, the precision of $\delta$ measurement as a function of the true value of $\delta$ for the three $s_{23}^{2}$ cases (high, mid and low). As expected, we have the overall best precision for the $s_{23}^{2}$ low case and the overall worst precision for the $s_{23}^{2}$ high case.

In the end we show the $1 \sigma$ and $2 \sigma$ region in the $\sin ^{2} \theta_{23}-\delta$ plane. These regions are calculated for different true values of $\delta=\left(-90^{\circ}, 0,90^{\circ}\right)$ and for $s_{23}^{2}=(0.441,0.5,0.587)$. To evaluate these contours we did not take into account previous knowledge about $s_{23}^{2}$, i.e. we have considered no pull term in the $\chi^{2}$ function. 


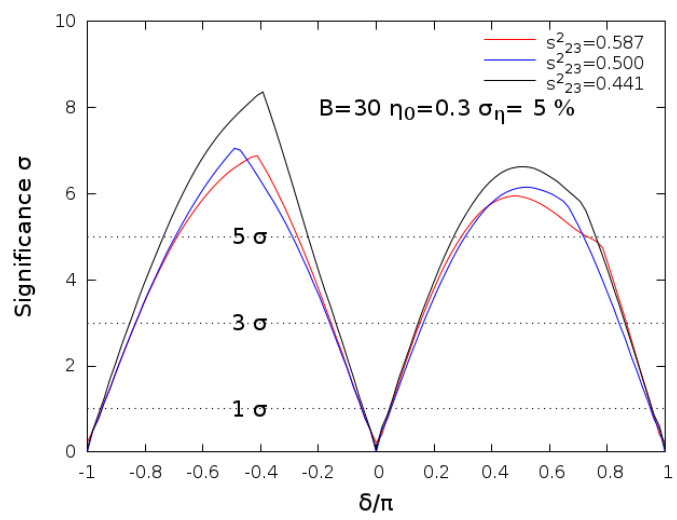

Figure 5.9: Expected significance in number of sigma to exclude $\delta=0, \pi$ for the combination $B=30$ and $\eta_{0}=0.3$ and $\sigma_{\eta}=5 \% \eta_{0}$ and for the three $s_{23}^{2}$ case (high,mid and low).

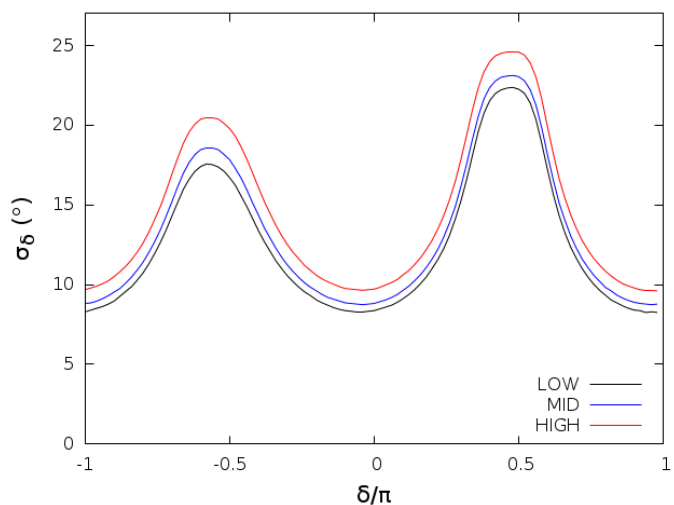

Figure 5.10: The $1 \sigma$ precision of the $\delta$ measurement as a function of the true $\delta$ value for the three $s_{23}^{2}$ cases (high, mid and low). Here we do not marginalize over $\eta$ and $s_{23}^{2}$.

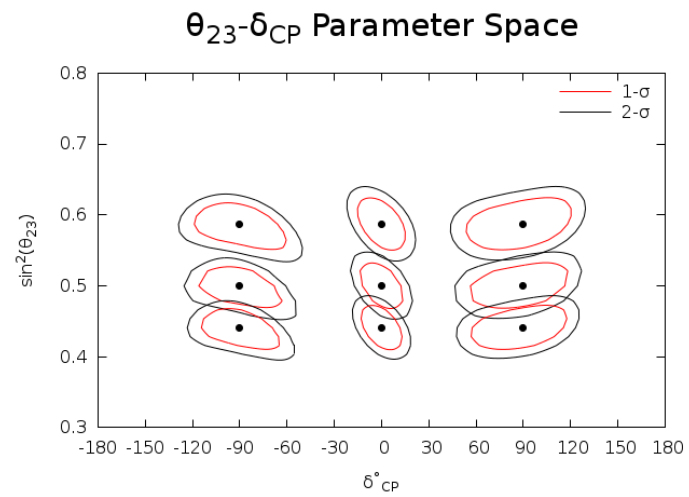

Figure 5.11: The expected $1 \sigma$ and $2 \sigma$ allowed regions in the $\sin ^{2} \theta_{23}-\delta$ plane. The results for the true values of $\delta=\left(-90^{\circ}, 0,90^{\circ}\right)$ and for $s_{23}^{2}=$ $(0.441,0.5,0.587)$ are shown. 


\section{6}

\section{Probing Neutrino Decay Scenarios with HK Detector}

As we have discussed previously, almost all the neutrino data are well explained by the $3 \nu$ model, i.e. the model that presents just three active neutrinos. This result itself is already the first proof of physics beyond the SM. It is then of interest to study more carefully if neutrino oscillations could give further information regarding to a more complete description of Nature. In this case, one would hope that measurements in neutrino experiments would eventually deviate from the expectations of the standard neutrino oscillation paradigm to some new physics. This could be possible with the next generation neutrino experiment such as Hyper-Kamiokande and its probable extension in Korea.

In this chapter we investigate how the $3 \nu$ model could be modified by neutrino decay scenario induced by non-diagonal couplings of Majorons $(\phi)$ to neutrinos. We will address the two possible types of decay known in literature as invisible and visible decay. We will define the decay parameter as $\alpha$ and the decay rate as $\Gamma$ where $\alpha_{i} \equiv \Gamma E_{\nu}$. We are interested to study the $\nu_{3}$ decay because on $\nu_{1}$ and $\nu_{2}$ decay there are strong limits coming from the solar data and Kamland experiments [37]. The most recent work has found $\alpha_{1}<3.4 \times 10^{-19} \mathrm{eV}^{2}$ and $\alpha_{2}=1.6 \times 10^{-13} \mathrm{eV}^{2}$.

For what it concerns the $\nu_{3}$ lifetime, there is still room for improvement. The actual limits we will refer to have been calculated for JUNO [38], DUNE [39] and ORCA [40], and they are $\alpha_{3}<8.8 \times 10^{-5} \mathrm{eV}^{2} 95 \%$ C.L., $\alpha_{3} \lesssim 6.0 \times 10^{-5} \mathrm{eV}^{2} 90 \%$ C.L. and $\alpha_{3}<2.6 \times 10^{-6} \mathrm{eV}^{2} 90 \%$ C.L. , respectively.

\section{1}

\section{Phenomenology of Neutrino Decay}

As a possible model of neutrino decay, we assume that neutrinos have following type of interaction(s) with a massless scalar, or so called Majoron $\phi$ [41],

$$
\mathscr{L}_{i n t}=\frac{1}{2}\left[\left(g_{s}\right)_{i j} \bar{\nu}_{i} \nu_{j} \phi+\mathrm{i}\left(g_{p}\right)_{i j} \bar{\nu}_{i} \gamma_{5} \nu_{j} \phi\right]
$$

where $g_{s}$ and $g_{p}$ implies, respectively, scalar and pseudo-scalar coupling and summation over the mass eigenstates are intended. In our work we will consider the case of the normal mass ordering (NO) $m_{1}<m_{2}<m_{3}$ and we will allow 
only $\nu_{3}$ state (and its antiparticle) to decay either into some sterile neutrino state (invisible decay) or into $\nu_{1}$ since the constraints on $\nu_{1}$ and $\nu_{2}$ coming from solar neutrino data are much stronger [42]. With these assumptions in mind we can write our evolution equation as follows

$\mathrm{i} \frac{\mathrm{d}}{\mathrm{d} x}\left(\begin{array}{l}\nu_{e} \\ \nu_{\mu} \\ \nu_{\tau}\end{array}\right)=\frac{1}{2 E_{\nu}}\left[U\left(\begin{array}{ccc}m_{1}^{2} & 0 & 0 \\ 0 & m_{2}^{2} & 0 \\ 0 & 0 & m_{3}^{2}-\mathrm{i} \alpha_{3}\end{array}\right) U^{\dagger}+\left(\begin{array}{ccc}2 \sqrt{2} E_{\nu} G_{F} N_{e}(x) & 0 & 0 \\ 0 & 0 & 0 \\ 0 & 0 & 0\end{array}\right)\right]\left(\begin{array}{c}\nu_{e} \\ \nu_{\mu} \\ \nu_{\tau}\end{array}\right)$

that coincides exactly with (2-65) except for the parameter $\alpha_{3}$ that has the dimension of $[E]^{2}$ describes the decay effect. It can be rewritten in terms of the decay rate of the $\nu_{3}$ neutrino mass eigenstate by the relation $\alpha_{3}=\Gamma_{3} E_{\nu}$ and also with respect to the $\nu_{3}$ lifetime as $\alpha_{3}=m_{3} / \tau_{3}$, where $\tau_{3}$ is the life time in the $\nu_{3}$ rest frame.

The constraints on the invisible decay can be obtained in a way that is model independent, this is, only in terms of a single parameter $\alpha_{3}$. On the other hand, constraints on the visible decay depends on other parameters such as the mass of $\nu_{1}$ and also we need to assume some type of coupling of neutrino with Majoron. Below we first discuss the case of invisible decay and then move to the case of visible decay. Unless otherwise stated, in this work, we consider the case of the normal mass ordering and the following values of the standard mixing parameters: $\Delta m_{31}^{2}=2.43 \times 10^{-3} \mathrm{eV}^{2}, \Delta m_{21}^{2}=7.5 \times 10^{-5} \mathrm{eV}^{2}$ , $s_{12}^{2}=0.31, s_{23}^{2}=0.5, s_{13}^{2}=0.023$.

\subsection{1}

\section{Invisible Decay}

As we have defined before, we will call invisible decay the process where $\nu_{3} \rightarrow \nu_{s}+\phi$, here $\nu_{s}$ is a sterile neutrino state and $\phi$ is the Majoron. In this case, to calculate the probability of oscillation plus decay effect for the arbitrary matter density profile, one can try to solve numerically the evolution equation (6-2) in order to compute the $\nu_{\alpha} \rightarrow \nu_{\beta}$ probability. In our case we will consider a constant matter profile so that we can find an analytic solution by simply replacing properly the relevant quantities in the vacuum case to that in matter plus decay case as follows

$$
P_{i n v}\left(\nu_{\alpha} \rightarrow \nu_{\beta}, E_{\nu}\right)=\left|\sum_{j=1}^{3} \tilde{U}_{\beta j} \tilde{U}_{j \alpha}^{-1} e^{-\mathrm{i} \frac{\tilde{m}_{j}^{2} L}{2 E_{\nu}}} e^{-\frac{\tilde{\alpha}_{3} L}{2 E_{\nu}}}\right|^{2},
$$

where $L$ is the distance traveled by neutrino, $\tilde{U}$ is the effective mixing matrix in matter in the presence of decay effect and all the eigenvalues of Hamiltonian 
with the tilde symbol are defined by the relations

$$
\tilde{U}^{-1} H\left(E_{\nu}\right) \tilde{U}=H_{\text {diag }},
$$

where

$$
H\left(E_{\nu}\right)=\left[U\left(\begin{array}{ccc}
m_{1}^{2} & 0 & 0 \\
0 & m_{2}^{2} & 0 \\
0 & 0 & m_{3}^{2}-\mathrm{i} \alpha_{3}
\end{array}\right) U^{\dagger}+\left(\begin{array}{ccc}
2 \sqrt{2} E_{\nu} G_{F} N_{e}(x) & 0 & 0 \\
0 & 0 & 0 \\
0 & 0 & 0
\end{array}\right)\right]
$$

and

$$
\left(H_{\text {diag }}\right)_{i i}=\tilde{m}_{i}^{2}-\mathrm{i} \tilde{\alpha}_{i} .
$$

It is important to note that the $\tilde{U}$ matrix is no longer a unitary matrix due to the presence of the decay term, and even if $\alpha_{1}=\alpha_{2}=0$ it is possible to have $\tilde{\alpha}_{i} \neq 0$ for $i=1,2$. For anti-neutrino, the same formula can be used by changing the signs of the matter potential and the $\mathrm{CP}$ phase.

Considering the case where the matter effect is small, from (6-3) we can roughly estimate the condition for a large decay effect, which is $O\left(\alpha_{3} L / E_{\nu}\right) \simeq 1$. This condition implies that in terms of $\alpha_{3}$,

$$
\alpha_{3} \sim \frac{E_{\nu}}{L} \sim 2 \times 10^{-4}\left[\frac{E_{\nu}}{\mathrm{GeV}}\right]\left[\frac{1000 \mathrm{~km}}{L}\right] \mathrm{eV}^{2},
$$

or equivalently, in terms of $\tau_{3} / m_{3}$

$$
\frac{\tau_{3}}{m_{3}}=\alpha_{3} \sim 3 \times 10^{-12}\left[\frac{E_{\nu}}{\mathrm{GeV}}\right]^{-1}\left[\frac{1000 \mathrm{~km}}{L}\right]^{-1} \frac{s}{\mathrm{eV}} .
$$

Below we discuss the impact of the decay effect for the disappearance and appearance channels separately.

\subsubsection{1}

\section{Disappearance modes $\nu_{\mu} \rightarrow \nu_{\mu}$ and $\bar{\nu}_{\mu} \rightarrow \bar{\nu}_{\mu}$}

Let us first consider the disappearance modes $\nu_{\mu} \rightarrow \nu_{\mu}$ and $\bar{\nu}_{\mu} \rightarrow \bar{\nu}_{\mu}$. In Fig. 6.1 we show $\nu_{\mu} \rightarrow \nu_{\mu}$ oscillation probabilities with and without decay effect in the left (right) panel for the baseline $L=295 \mathrm{~km}(1100 \mathrm{~km})$ as a function of the neutrino energy for $\alpha_{3}=5 \times 10^{-5} \mathrm{eV}^{2}$ (or $\tau_{3} / m_{3}=1.3 \times 10^{-11} \mathrm{~s} / \mathrm{eV}$ ). For $L=295 \mathrm{~km}(1100 \mathrm{~km})$ the matter density of $2.6 \mathrm{~g} / \mathrm{cm}^{3}\left(3.0 \mathrm{~g} / \mathrm{cm}^{3}\right)$ was used. We only show the case of the CP phase $\delta=-\pi / 2$ since the impact of decay on the probabilities with other values of $\delta$ give very similar results. We further note that the impact of decay on the probabilities for anti-neutrino channel $\bar{\nu}_{\mu} \rightarrow \bar{\nu}_{\mu}$ also give results very similar to that for the neutrino channel for the same decay parameter, hence we do not show them neither. We can see that for both baselines, the decay effect tends to reduce the probabilities or oscillatory behavior tends to be damped, see dashed (standard oscillation) and solid (with 

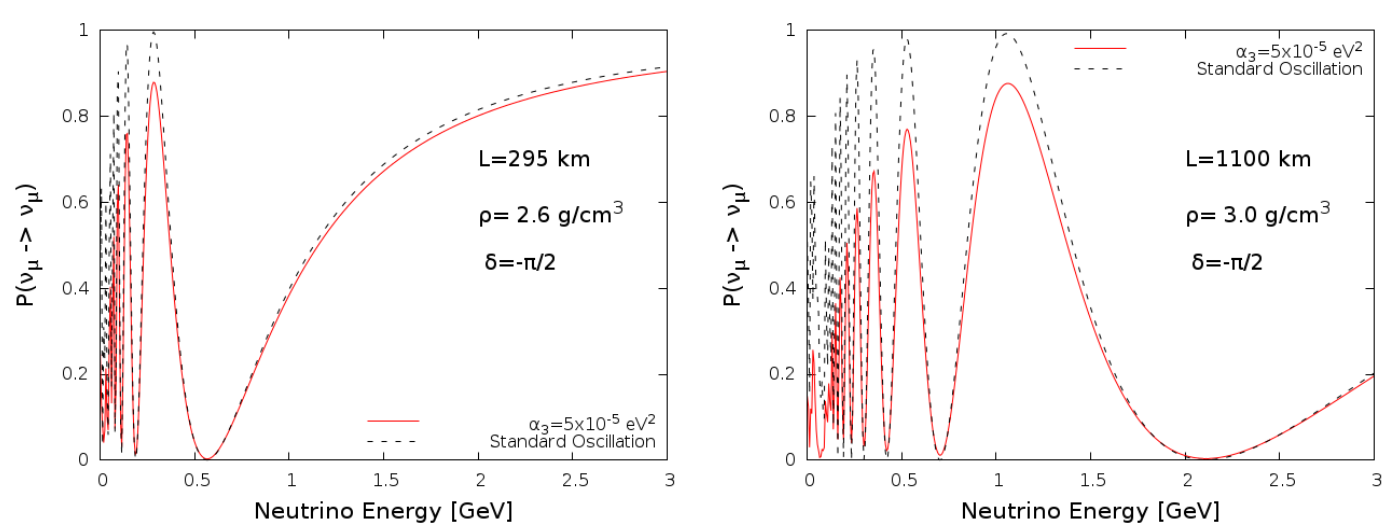

Figure 6.1: Probabilities for $\nu_{\mu} \rightarrow \nu_{\mu}$ disappearance channel with and without the effect of invisible decay as a function of the neutrino energy are shown for $\alpha_{3}=5 \times 10^{-5} \mathrm{eV}^{2}$ for $L=295 \mathrm{~km}$ (left panel) and $1100 \mathrm{~km}$ (right panel). The CP phase was fixed to $\delta=-\pi / 2$. The matter density of $2.6 \mathrm{~g} / \mathrm{cm}^{3}\left(3.0 \mathrm{~g} / \mathrm{cm}^{3}\right)$ were considered for $L=295 \mathrm{~km}(1100 \mathrm{~km})$.

decay effect) curves. We can also see the case of $L=1100 \mathrm{~km}$ has stronger impact of decay than the case of $L=295 \mathrm{~km}$, which is in agreement with the naive expectation since the former gives a larger value of $\alpha_{3} L / E_{\nu}$ for a given neutrino energy. We can also confirm that for a given baseline, the impact of decay is stronger for lower energy neutrinos, which is also expected from the energy dependence of the factor $\alpha_{3} L / E_{\nu}$ which controls the decay effect. Because of the large statistics for the disappearance modes, it is expected that the decay parameter considered in Fig. 6.1 would be easily probed (it can be excluded or confirmed) by T2HK/T2HKK experiments, which sensitivity will be extracted by our $\chi^{2}$ analysis.

\subsubsection{2}

Appearance modes $\nu_{\mu} \rightarrow \nu_{e}$ and $\bar{\nu}_{\mu} \rightarrow \bar{\nu}_{e}$

Let us now turn to the appearance modes $\nu_{\mu} \rightarrow \nu_{e}$ and $\bar{\nu}_{\mu} \rightarrow \bar{\nu}_{e}$. In Fig. 6.2 we show oscillation probabilities for $\nu_{\mu} \rightarrow \nu_{e}$ (left panels) and $\bar{\nu}_{\mu} \rightarrow \bar{\nu}_{e}$ (right panels) channel in the absence (dashed curves) and presence (solid curves) of the decay effect, in the upper (lower) panel for the baseline $L=295 \mathrm{~km}(1100 \mathrm{~km})$ as a function of the neutrino energy for $\alpha_{3}=5 \times 10^{-5} \mathrm{eV}^{2}$ (or $\tau_{3} / m_{3}=1.3 \times 10^{-11} \mathrm{~s} / \mathrm{eV}$ ). For $L=295(1100) \mathrm{km}$ and the matter density of $2.6 \mathrm{~g} / \mathrm{cm}^{3}\left(3.0 \mathrm{~g} / \mathrm{cm}^{3}\right)$ were used. We show the cases where $\delta= \pm \pi / 2,0$ and $\pi$.

By comparing the dashed curves (cases without decay effect) the solid curves (cases with decay effect) in Fig.6.2, we can see that the net effect of the decay is to reduce somewhat the oscillation probabilities or the damping of 

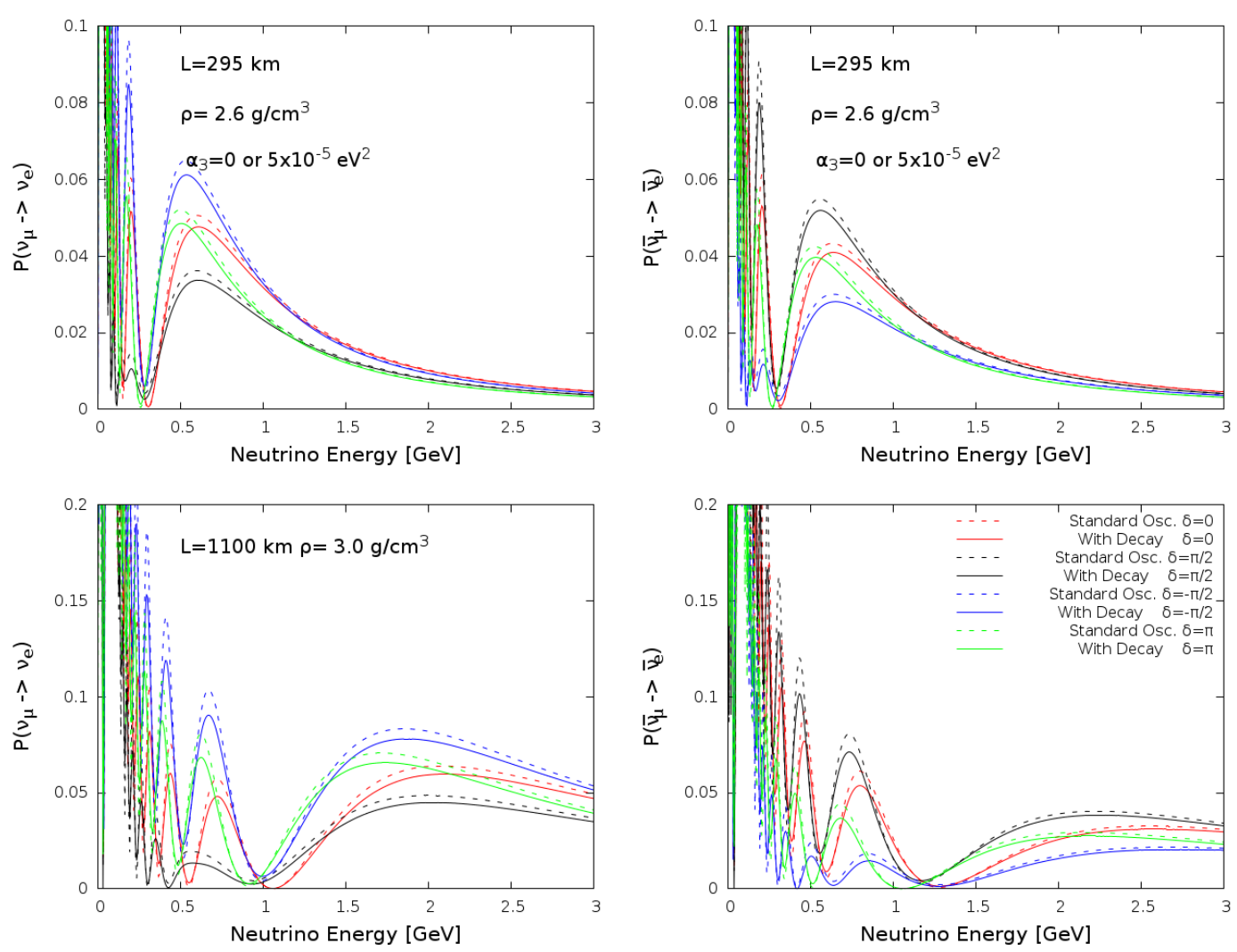

Figure 6.2: Probabilities for $\nu_{\mu} \rightarrow \nu_{e}$ (left panels) and $\bar{\nu}_{\mu} \rightarrow \bar{\nu}_{e}$ (right panels) channel in the absence and presence of the decay effect in the upper (lower) panel for the baseline $L=295 \mathrm{~km}(1100 \mathrm{~km})$ as a function of the neutrino energy for $\alpha_{3}=5 \times 10^{-5} \mathrm{eV}^{2}$ are shown. For $L=295$ (1100) $\mathrm{km}$ the matter density of $2.6 \mathrm{~g} / \mathrm{cm}^{3}\left(3.0 \mathrm{~g} / \mathrm{cm}^{3}\right)$ were used. We show the cases where $\delta= \pm \pi / 2,0$ and $\pi$.

the oscillation amplitudes as expected. We also note that if we compare the results for different baselines at the same energy, the impact of decay is stronger for $L=1100 \mathrm{~km}$ in agreement with the expectation. In contrast to the case of the disappearance modes, considering that the appearance channels have much less statistics, it seems that it would be difficult to exclude the cases shown in Fig.6.2. We will confirm this by our $\chi^{2}$ analysis.

\section{1 .2}

\section{Visible Decay}

Let us now turn to the case of visible decay. In this work, following recent works $[39,43]$ we consider the case where $\nu_{3}$ decays into $\nu_{1}$ plus Majoron $(\phi)$. In contrast to the case of invisible decay, to discuss the phenomenology of the visible decay, we need to take into account the energy spectrum of the initial neutrino flux, which is, in our case $\nu_{\mu}\left(\bar{\nu}_{\mu}\right)$ flux coming from the J-PARC Off Axis beams. Let us consider the oscillation channel of $\nu_{\alpha}^{A} \rightarrow \nu_{\beta}^{B}$ where $\alpha$ and 
$\beta$ indicate flavor (in our case, $\alpha=\mu, \beta=e$ ) while $\mathrm{A}$ and $\mathrm{B}$ indicate neutrino helicity, - for neutrino or + for anti-neutrino. Since the model we consider allows neutrinos to decay into different helicities, in this work we will take this effect into account following [43]. Then the energy spectrum of the $\nu_{\beta}^{B}$ arriving at the detector located at the baseline of $L$ can be expressed as

$$
\frac{\mathrm{d} \phi_{\nu_{\beta}^{B}}^{\text {osc }+ \text { decay }}\left(E_{\nu_{\beta}^{B}}\right)}{\mathrm{d} E_{\nu_{\beta}^{B}}}=\int \mathrm{d} E_{\nu_{\alpha}^{A}} P_{\alpha \beta}^{\text {osc }+ \text { decay }}\left(E_{\nu_{\alpha}^{A}}, E_{\nu_{\beta}^{B}}\right) \frac{\mathrm{d} \phi_{\nu_{\alpha}^{A}}^{0}\left(E_{\nu_{\alpha}^{A}}\right)}{\mathrm{d} E_{\nu_{\alpha}^{A}}}
$$

where $\mathrm{d} \phi_{\nu_{\alpha}^{A}}^{0}\left(E_{\nu_{\alpha}^{A}}\right) / \mathrm{d} E_{\nu_{\alpha}^{A}}$ is the neutrino flux spectrum expected at the detector site in the absence of any oscillation and decay effect and

$$
\begin{aligned}
P_{\alpha \beta}^{\text {osc }+ \text { decay }}\left(E_{\nu_{\alpha}^{A}}, E_{\nu_{\beta}^{B}}\right) & =P_{i n v}\left(\nu_{\alpha}^{A} \rightarrow \nu_{\beta}^{B} ; E_{\nu_{\alpha}^{A}}\right) \delta_{A B} \delta\left(E_{\nu_{\alpha}^{A}}-E_{\nu_{\beta}^{B}}\right) \\
& +\frac{\mathrm{d} P^{\nu_{\alpha}^{A} \rightarrow \nu_{\beta}^{B}}\left(E_{\nu_{\alpha}^{A}}, E_{\nu_{\beta}^{B}}\right)}{\mathrm{d} E_{\nu_{\beta}^{B}}}
\end{aligned}
$$

where the first term in (Fig. 6-10) coincides with the one given in Eq. (6-3) for the invisible decay, which is nonzero only for the same energy and helicity of neutrino. As described in the Appendix B, the second term in (Fig. 6-10) can be expressed as

$$
\frac{\mathrm{d} P^{\nu_{\alpha}^{A} \rightarrow \nu_{\beta}^{B}}\left(E_{\nu_{\alpha}}, E_{\nu_{\beta}}\right)}{\mathrm{d} E_{\nu_{\beta}}}=Q_{\alpha \beta}^{A B}\left(E_{\nu_{\alpha}}, E_{\nu_{\beta}}\right) \frac{\mathrm{d}}{\mathrm{d} E_{\nu_{\beta}}} \Gamma_{\nu_{3}^{A} \rightarrow \nu_{1}^{B}}\left(E_{\nu_{\alpha}}, E_{\nu_{\beta}}\right),
$$

where the expressions of the functions $Q_{\alpha \beta}^{A B}\left(E_{\nu_{\alpha}}, E_{\nu_{\beta}}\right)$ and $\mathrm{d} \Gamma_{\nu_{3}^{A} \rightarrow \nu_{1}^{B}}\left(E_{\nu_{\alpha}^{A}}, E_{\nu_{\beta}^{B}}\right) / \mathrm{d} E_{\nu_{\beta}^{B}}$ are given, respectively, in Eq.(B-4) and Eq.(B-7) in Appendix B. See also Figs. B.2 and B.1 in Appendix which show, respectively, the energy dependence of these functions. We note that compared to the case of invisible decay, the probability for the visible decay is always equal or larger as the quantity given in Eq. (6-11) is non-negative. Compared to the case of invisible decay, impact of the contribution from the visible decay, described by (6-11), is somewhat more complicated as the initial neutrino $\nu_{\alpha}\left(\right.$ or $\left.\bar{\nu}_{\alpha}\right)$ with a given energy $E_{\nu_{\alpha}}$ (or $E_{\bar{\nu}_{\alpha}}$ ), in general, arrive at the detector as $\nu_{\beta}$ and $\bar{\nu}_{\beta}$ with different energies, $E_{\nu_{\beta}}$ and $E_{\bar{\nu}_{\beta}}$, respectively. While we should look at the expected distributions in the number of events taking into account properly neutrino helicity (or the dependence of cross sections on neutrino helicity), in order to have some feeling regarding the magnitude of the impact of visible decay on oscillation probabilities, let us define the following "effective" probability,

$$
P_{\text {vis }}^{\text {eff }}\left(\nu_{\alpha} \rightarrow \nu_{\beta} ; E_{\nu_{\beta}}\right) \equiv \frac{\frac{\mathrm{d} \phi_{\nu_{\beta}}^{\text {osc+decay }}}{\mathrm{d} E_{\nu_{\beta}}}+\frac{\mathrm{d} \phi_{\bar{\nu}_{\beta}}^{\text {osc+decay }}}{\mathrm{d} E_{\bar{\nu}_{\beta}}}}{\frac{\mathrm{d} \phi_{\nu_{\alpha}^{A}}^{0}\left(E_{\nu_{\beta}}\right)}{\mathrm{d} E_{\nu_{\beta}}}} .
$$


This is just the sum of the probabilities given in (6-10) over different neutrino helicities, averaged over the initial neutrino flux spectrum, so we can also write

$$
P_{v i s}^{e f f}\left(\nu_{\alpha} \rightarrow \nu_{\beta} ; E_{\nu_{\beta}}\right)=\left\langle P_{\alpha \beta}\left(E_{\nu_{\alpha}}, E_{\nu_{\beta}}\right)\right\rangle_{\mathrm{d} \phi^{0} / \mathrm{d} E_{\nu_{\beta}}} .
$$

Note that the probability defined in (6-12) can, in principle, exceed 1 for a given neutrino energy as parent neutrinos with different energy can contribute to the daughter neutrino with some given energy accumulatively. Let us stress that this function depends on the energy dependence of the initial neutrino flux at the source. For the computation defined in (6-12) what is relevant is only the energy dependence of the initial neutrino flux (all the other factors in the flux cancel out) and in the case there is no visible decay contribution, probability given in (6-12) coincides with the invisible decay probability given in (6-3). For the baseline $\mathrm{L}=295 \mathrm{~km}(1100 \mathrm{~km})$ for T2HK (T2HKK) experiment we use the $2.5^{\circ}\left(2.5^{\circ}\right)$ off axis neutrino beam from J-PARC taken from [16] ([24]).

\subsubsection{1}

\section{Disappearance modes $\nu_{\mu} \rightarrow \nu_{\mu}$ and $\bar{\nu}_{\mu} \rightarrow \bar{\nu}_{\mu}$}

As in the case of invisible decay, let us first discuss the impact of visible decay for the disappearance modes $\nu_{\mu} \rightarrow \nu_{\mu}$ and $\bar{\nu}_{\mu} \rightarrow \bar{\nu}_{\mu}$. In Fig.6.3 we show the similar plots shown in Fig. 6.1 by adding one more curve, this was computed by using the effective probability with the visible decay effect, as defined in (6-12), and using the same decay parameter $\alpha_{3}=5 \times 10^{-5} \mathrm{eV}^{2}$ used for invisible decay shown in Fig.6.1. As in the case of invisible decay, the impact of varying the $\mathrm{CP}$ phase as well as the difference between neutrino and antineutrino channels are very small, hence we show only the case with $\delta=-\pi / 2$ for neutrino channel. By comparing the solid red curve and dashed blue curve in Fig.6.3, we see that the difference between the cases of invisible and visible decays is not significant. We observe that the difference is large only in the lower energy part $(<0.5 \mathrm{GeV})$ of the probability which suffers rapid oscillation. We expect that the disappearance channel give similar sensitivities to the decay parameter $\alpha_{3}$ to that to be obtained by the invisible decay case.

\subsubsection{2}

Appearance modes $\nu_{\mu} \rightarrow \nu_{e}$ and $\bar{\nu}_{\mu} \rightarrow \bar{\nu}_{e}$

Let us now discuss the case of appearance modes, $\nu_{\mu} \rightarrow \nu_{e}$ and $\bar{\nu}_{\mu} \rightarrow \bar{\nu}_{e}$. In Fig. 6.4 we show the effective probabilities computed by using the definition (6-12) as a function of the neutrino energy in the upper and lower 2 panels, respectively, for $L=295 \mathrm{~km}$ and $L=1100 \mathrm{~km}$, for $\alpha_{3}=2 \times 10^{-5} \mathrm{eV}^{2}$, and the mass ratios of $m_{3} / m_{1}=1.2,2.0$ and 100 for the case where only the scalar 

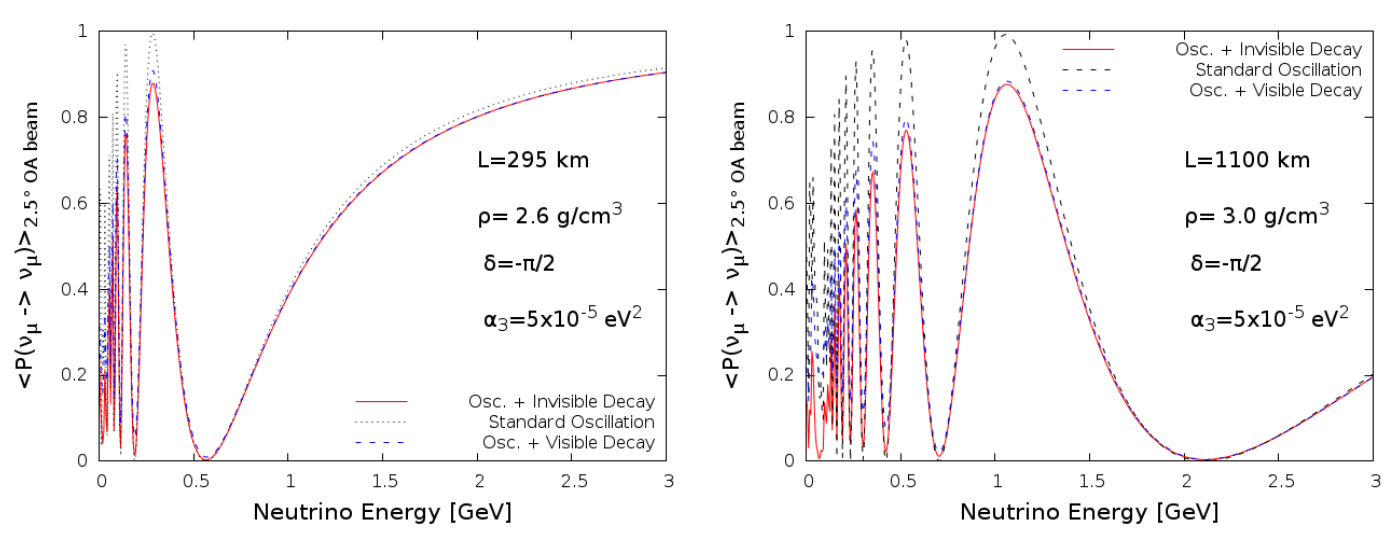

Figure 6.3: Probabilities for $\nu_{\mu} \rightarrow \nu_{\mu}$ disappearance channel with and without invisible decay as a function of the neutrino energy are shown for $\alpha_{3}=$ $5 \times 10^{-5} \mathrm{eV}^{2}$ for $L=295 \mathrm{~km}$ (left panel) and $1100 \mathrm{~km}$ (right panel). The CP phase was fixed to $\delta=-\pi / 2$. The matter density of $2.6 \mathrm{~g} / \mathrm{cm}^{3}\left(3.0 \mathrm{~g} / \mathrm{cm}^{3}\right)$ were considered for $L=295 \mathrm{~km}(1100 \mathrm{~km})$. The case for the visible decay indicated by the dashed blue curves were computed by using the formula defined in (6-12).

coupling is nonzero. The dependence of the mass ratio on probabilities comes from the part $\mathrm{d} \Gamma_{\nu_{3} \rightarrow \nu_{1}} / \mathrm{d} E(\mathrm{~B}-7)$. For $L=295 \mathrm{~km}(L=1100 \mathrm{~km})$ we used the energy spectrum of $2.5^{\circ}\left(2.5^{\circ}\right)$ Off Axis neutrino beams from J-PARC taken from [16] ([24]), to compute the average (effective) probabilities.

For the appearance modes, the impact of visible decay is much larger, therefore we considered smaller value of $\alpha_{3}$. Since we are considering the sum of $\nu+\bar{\nu}$ in (6-12) the corresponding plot (for the case where only the pseudoscalar coupling is nonzero) is very similar to that shown in Fig. 6.4 hence we do not show the plot for other coupling here. See, however, Fig. 6.5 and text below to see the different impact on individual probabilities for $\nu$ and $\bar{\nu}$ due to the difference of the type of couplings.

As we can see, the impact of visible decay on the appearance channels is much larger compared to the case of invisible decay, in particular for antineutrino channels, in agreement with the discussions given in [43] for their T2K analysis. We note that for $\bar{\nu}_{\mu} \rightarrow \bar{\nu}_{e}$ mode, some fraction of neutrinos arriving at the detector contribute as neutrino due to the helicity non-conserving decay, which in general increase the excess of the number of events because the cross section for neutrino is larger than that for anti-neutrino. However, being the Water Cherenkov detector, HK can not distinguish neutrino from anti-neutrino event by event basis. Therefore, we will treat events coming from $\nu_{e}$ and $\bar{\nu}_{e}$ $\left(\nu_{\mu}\right.$ and $\left.\bar{\nu}_{\mu}\right)$ as if they were all coming from $\nu_{e}\left(\nu_{\mu}\right)$ and $\bar{\nu}_{e}\left(\bar{\nu}_{\mu}\right)$, respectively, for the $\nu$ and $\bar{\nu}$ running mode. In order to see more clearly the contributions of each helicity for the visible decay, for the cases of scalar and pseudo-scalar 

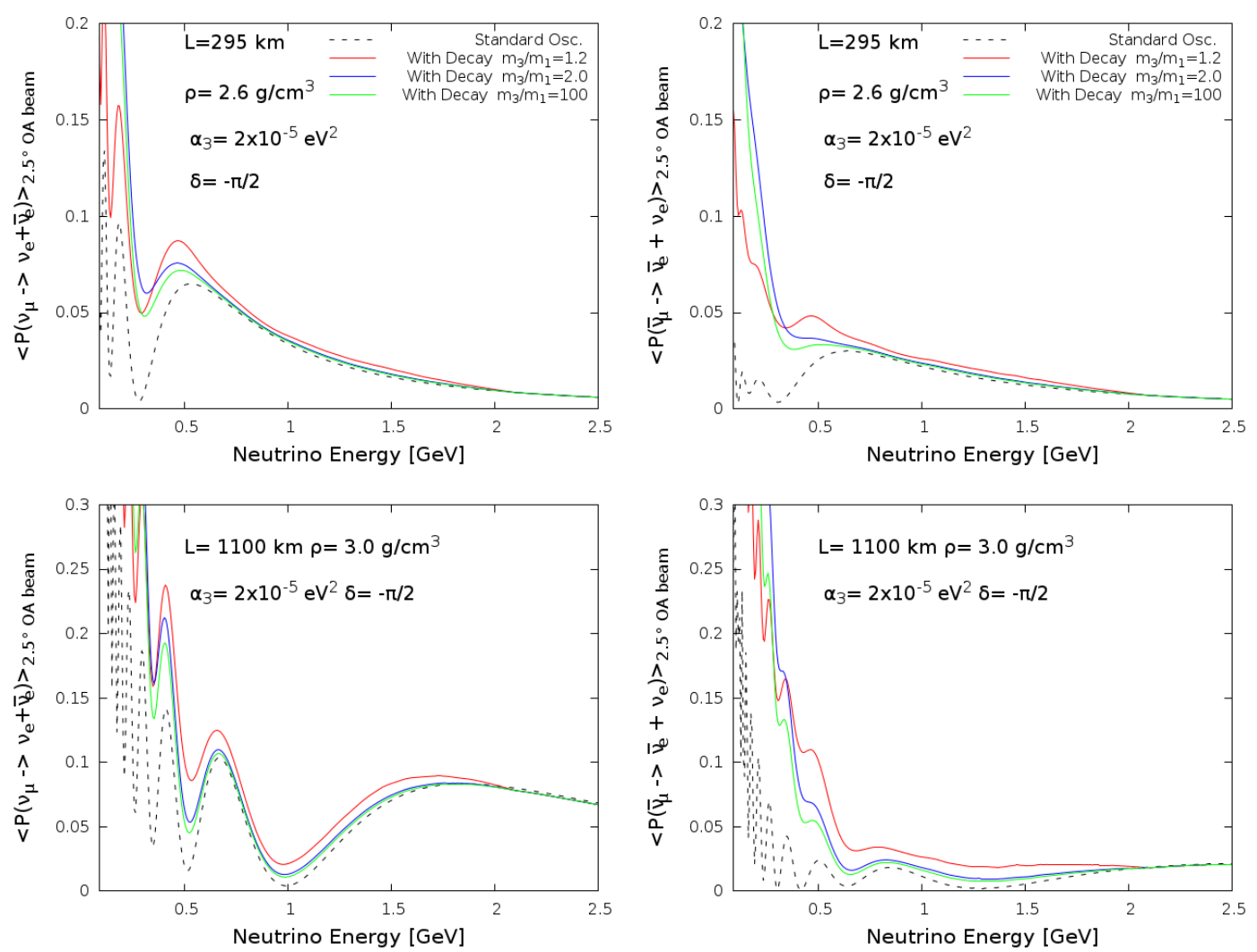

Figure 6.4: Effective probabilities for $\nu_{\mu} \rightarrow \nu_{e}$ (left panels) and $\bar{\nu}_{\mu} \rightarrow \bar{\nu}_{e}$ (right panels) defined in Eq.(6-12) as a function of the neutrino energy are shown, in the upper and lower 2 panels, respectively, for $L=295 \mathrm{~km}$ and $L=1100 \mathrm{~km}$, for $\alpha_{3}=2 \times 10^{-5} \mathrm{eV}^{2}$, and the mass ratios of $m_{3} / m_{1}=1.2,2.0$ and 100 . The matter density of $2.6 \mathrm{~g} / \mathrm{cm}^{3}\left(3.0 \mathrm{~g} / \mathrm{cm}^{3}\right)$ were considered for $L=295 \mathrm{~km}(1100$ $\mathrm{km})$.

coupling, we show in Fig. 6.5 only the contributions from the visible decay in the probabilities defined by

$$
\Delta P\left(\nu_{\mu} \rightarrow \nu_{e} ; E_{\nu_{e}}\right)=P_{e f f}^{o s c+\text { decay }}\left(\nu_{\mu} \rightarrow \nu_{e} ; E_{\nu_{e}}\right)-P_{i n v}\left(\nu_{\mu} \rightarrow \nu_{e} ; E_{\nu_{e}}\right),
$$

for each helicity mode for the case for $\alpha_{3}=2 \times 10^{-5} \mathrm{eV}^{2}$ and the mass ratio $m_{3} / m_{1}=2.0$ for the purpose of illustration.

As we can see in Fig. 6.5, the impact of visible decay is significant only for the neutrino energies smaller than $\sim 0.6(1.2) \mathrm{GeV}$ for the baseline $\mathrm{L}=295$ $\mathrm{km}(1100 \mathrm{~km})$. For the both neutrino and anti-neutrino modes, shown in Fig. 6.5 , we can see that the helicity conserving (non-conserving) decay is dominant for the case of scalar (pseudo-scalar) coupling.

\section{2}

\section{Experimental setup and assumptions for our analysis}



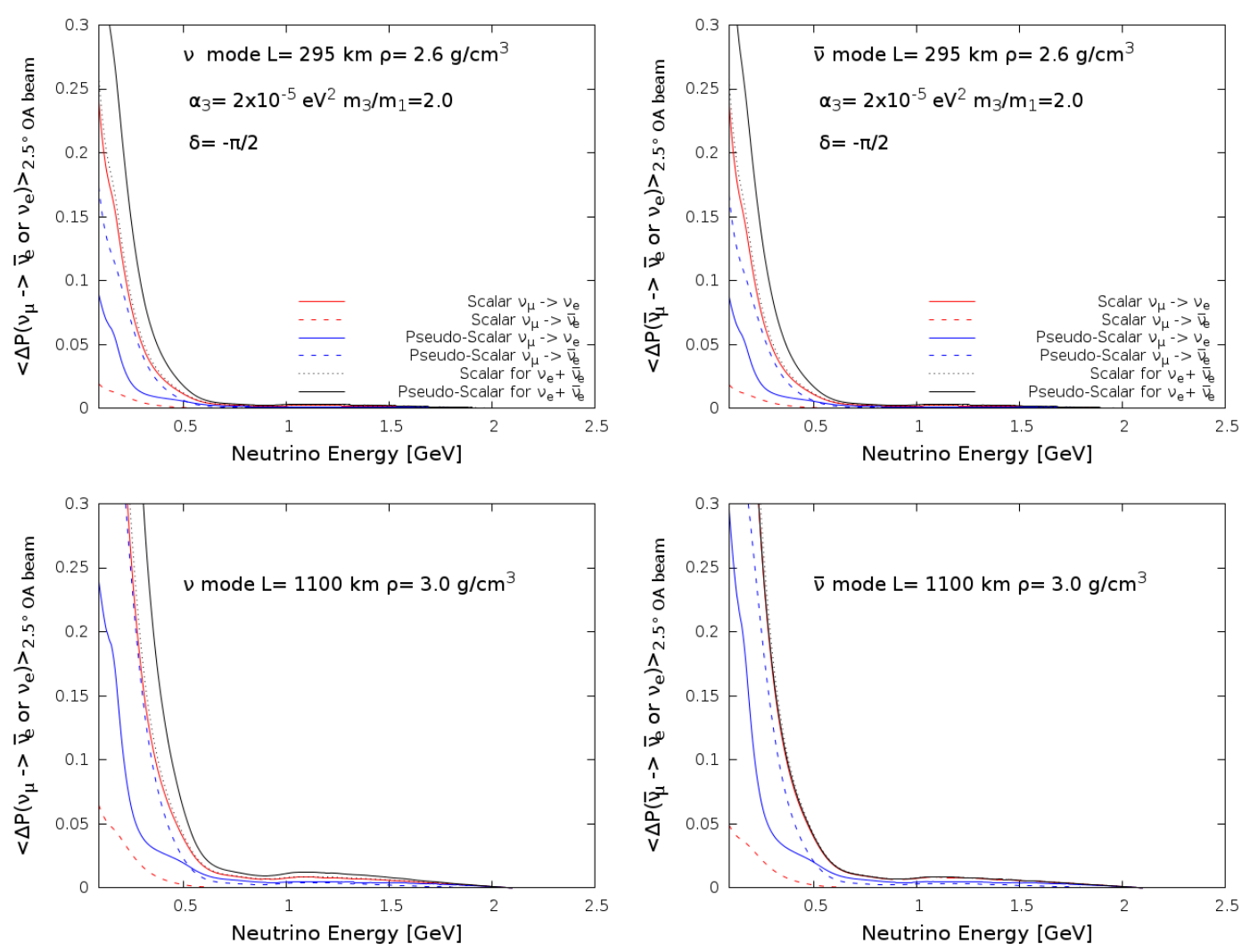

Figure 6.5: Contribution of each helicity in the visible decay, in $\Delta P_{\mu e}=$ $P_{e f f}^{o s c+d e c a y}\left(\nu_{\mu} \rightarrow \nu_{e} ; E_{\nu_{e}}\right)-P_{i n v}\left(\nu_{\mu} \rightarrow \nu_{e} ; E_{\nu_{e}}\right)$ as a function of the neutrino energy are shown, in th upper and lower 2 panels, respectively, for $\mathrm{L}=295 \mathrm{~km}$ and $\mathrm{L}=1100 \mathrm{~km}$, for $\alpha_{3}=2 \times 10^{-5} \mathrm{eV}^{2}$, and the mass ratios of $m_{3} / m_{1}=2.0$. The matter density of $2.6 \mathrm{~g} / \mathrm{cm}^{3}\left(3.0 \mathrm{~g} / \mathrm{cm}^{3}\right)$ were considered for $L=295 \mathrm{~km}$ $(1100 \mathrm{~km})$.

\subsection{1}

\section{Hyper-Kamiokande}

Hyper-Kamiokande (HK) [16] is the successor of the Super-Kamiokande [44] in Japan. It is the next generation Water Cherenkov detector to be located in Tochibora mine at $295 \mathrm{~km}$ away from the J-PARC proton accelerator research complex in Tokai, Japan. One of the important physics programs of $\mathrm{HK}$ is the long-baseline neutrino oscillation experiment aim to observe $\mathrm{CP}$ violation in the lepton sector. Hyper-K will adopt the staging approach: the two cylindrical detectors with the same size (60 $\mathrm{m}$ height and $74 \mathrm{~m}$ in diameter) and $40 \%$ photocoverrage will be constructed with some time interval. The first detector (tank) will be build in Tochibora mine in Japan. The construction of the first detector is expected to start in 2020. We consider its default setup for HK experiment described in the HK design report [16]. The fiducial volume of the 1 tank is assumed to be $187 \mathrm{kt}$ (with the total mass is $258 \mathrm{kt}$ ). For the 

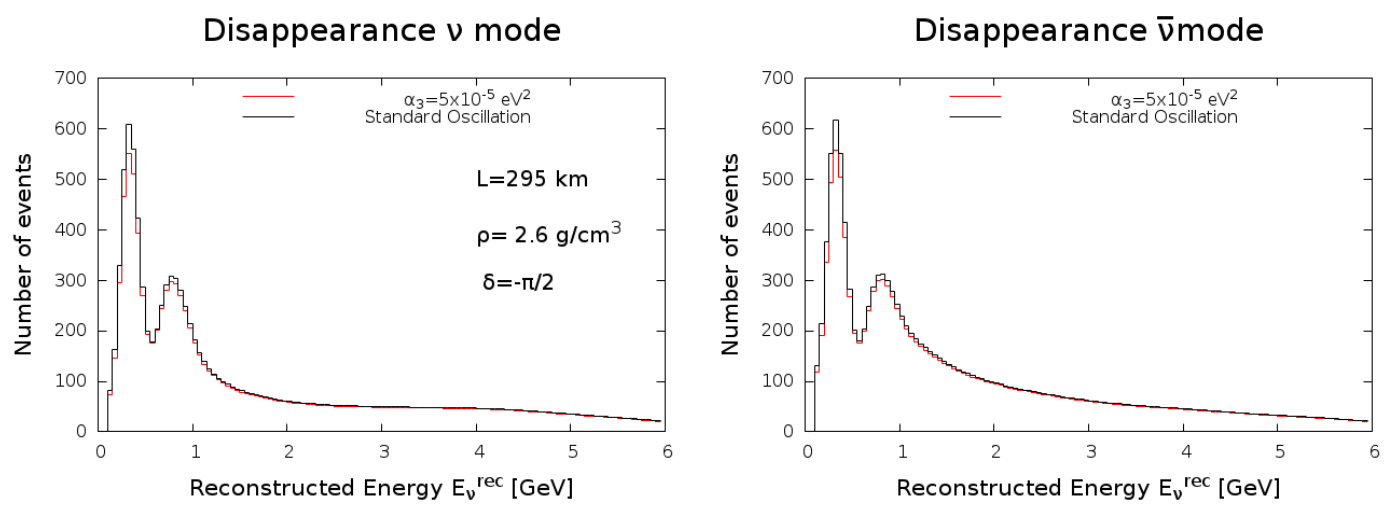

Figure 6.6: Expected events number distribution for the disappearance channel for T2HK for $\nu_{\mu} \rightarrow \nu_{\mu}$ (left panel) and $\bar{\nu}_{\mu} \rightarrow \bar{\nu}_{\mu}$ (right panel).

neutrino beam, we assume the 2.5 degree off axis neutrino beam from J-PARC taken from [16]. For brevity, we call this experimental setup "T2HK" (Tokai to Hyper- Kamokande). For T2HK, we assume the total running time of 10 years with $1.3 \mathrm{MW} \times 10^{7}$ second with the beam running mode time ratio for neutrino and anti-neutrino of 1:3 (a longer run for $\bar{\nu}$ mode is needed because the expected flux and cross section are smaller for $\bar{\nu}$ ). We compute the expected number of events for disappearance modes, $\nu_{\mu} \rightarrow \nu_{\mu}$ and $\bar{\nu}_{\mu} \rightarrow \bar{\nu}_{\mu}$ as well as for the appearance modes $\nu_{\mu} \rightarrow \nu_{e}$ and $\bar{\nu}_{\mu} \rightarrow \bar{\nu}_{e}$ in the presence of the decay effect. We describe the details of our calculations in Appendix C. In Fig. 6.6 we show the example of event number distribution of the disappearance modes, $\nu_{\mu} \rightarrow \nu_{\mu}$ (left panel) and $\bar{\nu}_{\mu} \rightarrow \bar{\nu}_{\mu}$ (right panel) for the T2HK experiment with and without invisible decay effect. As we expected from the discussion in the previous section (6.1.1) we confirm that the net effect of the decay is to reduce somewhat the signal compared to the case of the standard oscillation without any decay effect.

In Fig. 6.7 we show the similar plots as in Fig. 6.6 but for the appearance modes, $\nu_{\mu} \rightarrow \nu_{e}$ (left panel) and $\bar{\nu}_{\mu} \rightarrow \bar{\nu}_{e}$ (right panel) for the T2HK experiment. In the case of invisible decay, the qualitative behavior is similar to the case of disappearance modes, namely, the reduction of the signal compared to the case of the standard oscillation.

On the other hand, let us see what is the effect of the visible decay on both channel for T2HK. In Fig. 6.11 we show the effect of the scalar coupling helicity conserving visible decay process on disappearance channel $\nu_{\mu} \rightarrow \nu_{e}$ (left panel) and $\bar{\nu}_{\mu} \rightarrow \bar{\nu}_{e}$ (right panel) compared to the invisible decay case and the standard oscillation case. As we have discussed before, we can see how the visible case adds events in the bin $\lesssim 0.7 \mathrm{GeV}$ and it always is bigger than the invisible case. Even if for both decays we use the same decay parameter 

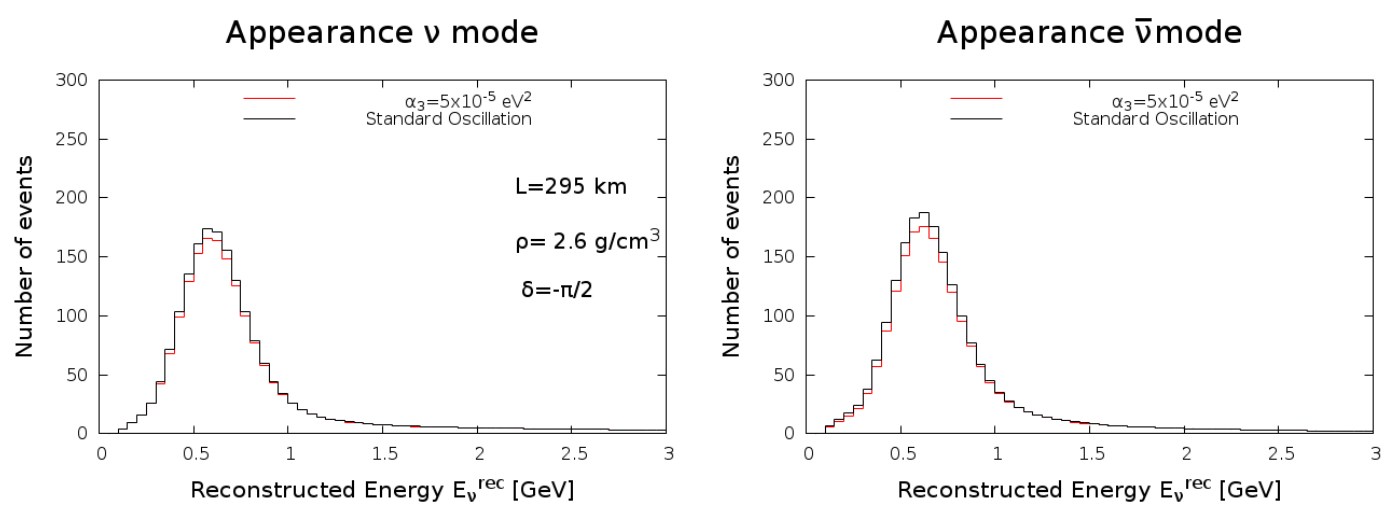

Figure 6.7: Expected events number distribution for the disappearance channel for T2HK for $\nu_{\mu} \rightarrow \nu_{e}$ (left panel) and $\bar{\nu}_{\mu} \rightarrow \bar{\nu}_{e}$ (right panel) for the case of standard oscillation against invisible decay effect with $\alpha_{3}=5 \times 10^{-5} \mathrm{eV}^{2}$.

$\alpha_{3}$ we can see that the visible case has a bigger effect than the invisible one. Indeed, we expect a higher sensitivity in this channel for the visible effect.

\section{2 .2}

\section{Second Hyper-Kamiokande Detector in Korea}

There is a possibility that the second detector which is supposed to have the same size as the first detector will be placed somewhere in Korea [24] such that the baseline is $\sim L=1100 \mathrm{~km}$. While this possibility is still under discussion and we do not know the details of the precise location of the detector, for the sake of discussion, in this work we assume that the detector will be located at the baseline $L=1100 \mathrm{~km}$ from J-PARC and receive the $2.5^{\circ}$ off axis neutrino beam. Despite that there will be some time difference between the data taking of the first HK detector in Japan and the second one in Korea, for simplicity, we assume the same beam power, running time and the time ratio for neutrino and anti-neutrino mode also for the second detector in Korea. For brevity we call this setup as "T2HKK" (Tokai to Hyper-K and Korea). In Fig. 6.9 we show an example of expected distributions for the number of events in the disappearance channels for T2HKK for $\nu_{\mu} \rightarrow \nu_{\mu}$ (left panel) and $\bar{\nu}_{\mu} \rightarrow \bar{\nu}_{\mu}$ (right panel) with and without invisible decay effect.

In Fig. 6.10 instead we show a similar plot for the case of the disappearance channel. For the visible decay we show the effect in Fig. 6.11.

\section{3}

\section{Definition of $\chi^{2}$ Function}

Here we are going to present how we define our $\chi^{2}$ function that we have used in our statistical analysis. We choose to use a Gaussian $\chi^{2}$ function: 

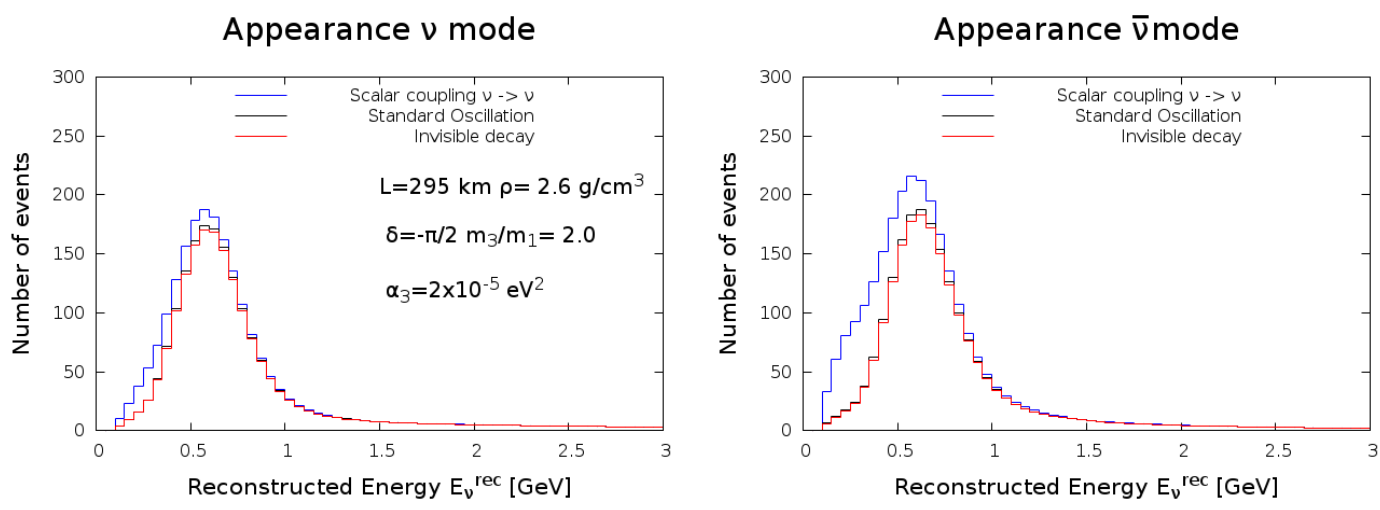

Figure 6.8: Expected events number distribution for the appearance channel for T2HK for $\nu_{\mu} \rightarrow \nu_{e}$ (left panel) and $\bar{\nu}_{\mu} \rightarrow \bar{\nu}_{e}$ (right panel) for the case of standard oscillation against invisible decay and scalar coupling helicity conserving visible decay effect. For both cases we used $\alpha_{3}=5 \times 10^{-5} \mathrm{eV}^{2}$ and for the visible case the mass ratio $m_{3} / m_{1}=2.0$.

$$
\chi^{2}=\chi_{\text {stat }}^{2}+\chi_{\text {pull }}^{2}
$$

where $\chi_{\text {stat }}^{2}$ is defined as:

$$
\chi^{2}\left(m_{\text {light }}, \Delta m_{31}^{2}, \sin _{23}^{2}, \delta_{C P}, \alpha_{3}, \beta\right) \equiv \sum_{e=f, n}\left[\sum_{i=1}^{\text {bins }}\left\{\frac{\left(N_{e, i}^{\mathrm{obs}}-\beta N_{e, i}^{\mathrm{fit}}\right)^{2}}{N_{e, i}^{\mathrm{fit}}+\sigma_{\mathrm{sys}}\left(N_{e, i}^{\mathrm{fit}}\right)^{2}}\right\}\right],
$$

where $N_{e, i}^{\mathrm{obs}}$ and $N_{e, i}^{\mathrm{fit}}$ are, respectively, the observed (simulated) and the fitted (theoretical expected) number of events in the $i-$ th energy bin. We also introduce $\sigma_{\text {sys }}^{2}$ and we define it as

$$
\sigma_{\text {sys }}^{2}=f \cdot\left(N_{e, i}^{\mathrm{obs}}\right)^{2}
$$

This parameter $f$ takes into account the specific experiment systematic errors. In our case we choose a value for this parameter of $f=0.03$. Here the subindex $e=f, n$ denote the two experiments we are consider, first T2HKK in Korea as $f$ (far) detector with $1100 \mathrm{~km}$ baseline and the second experiment as $n$ (near) which is T2HK located at kamioka and $295 \mathrm{~km}$ baseline. In the case of the combined analysis we will add together the two contributions to compute $N_{e, i}^{\text {obs }}$ we assume the input values of oscillation parameters as follows as we showed 

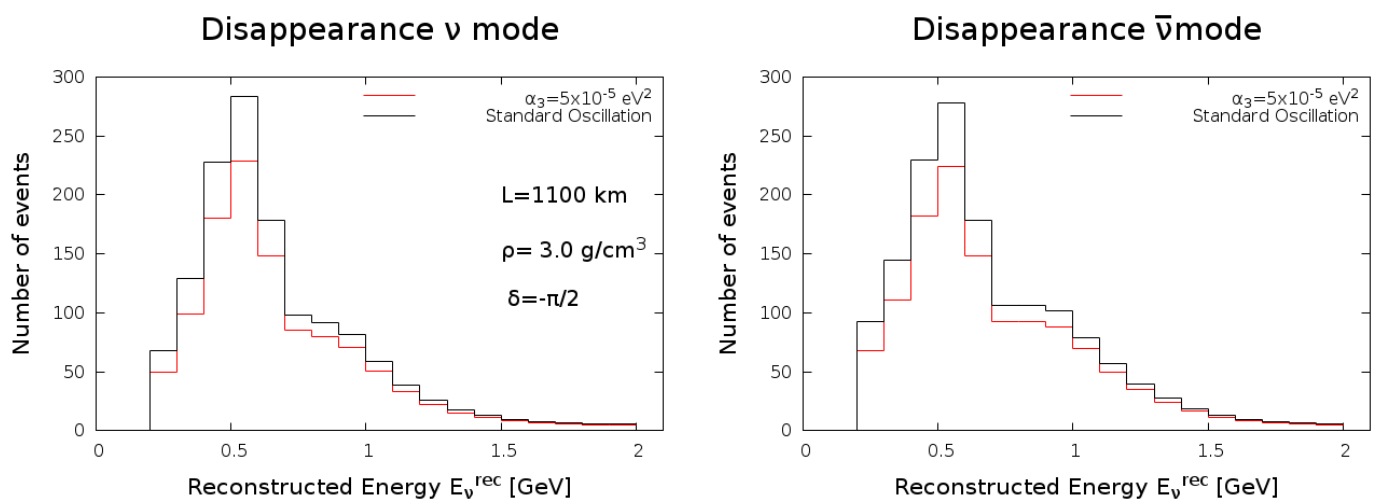

Figure 6.9: Expected events number distribution for the disappearance channel for T2HKK for $\nu_{\mu} \rightarrow \nu_{\mu}$ (left panel) and $\bar{\nu}_{\mu} \rightarrow \bar{\nu}_{\mu}$ (right panel) for the case of standard oscillation against invisible decay effect with $\alpha_{3}=5 \times 10^{-5} \mathrm{eV}^{2}$.
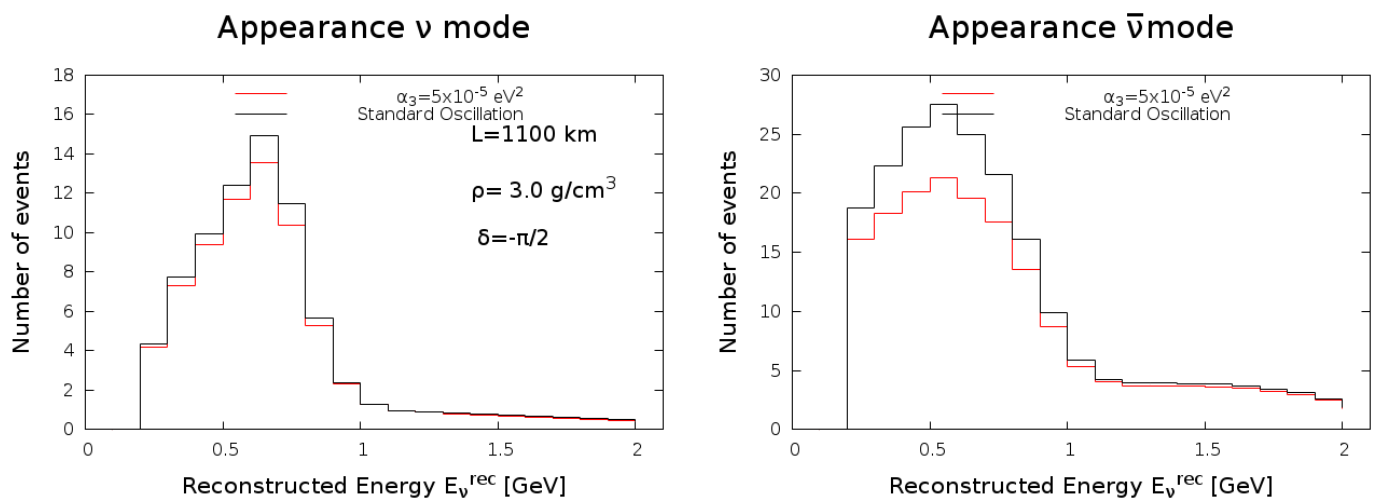

Figure 6.10: Expected events number distribution for the appearance channel for T2HKK for $\nu_{\mu} \rightarrow \nu_{e}$ (left panel) and $\bar{\nu}_{\mu} \rightarrow \bar{\nu}_{e}$ (right panel) for the case of standard oscillation against invisible decay effect with $\alpha_{3}=5 \times 10^{-5} \mathrm{eV}^{2}$.

in the previous section

$$
\begin{aligned}
m_{\text {light }} & =1.0 \times 10^{-3} \mathrm{eV} \\
\Delta m_{31}^{2} & =2.43 \times 10^{-3} \mathrm{eV}^{2} \\
\sin _{23}^{2} & =0.5 \\
\delta_{C P} & =-\pi / 2 \\
\alpha_{3} & =1.0 \times 10^{-6} \mathrm{eV}^{2} .
\end{aligned}
$$

The $\beta$ parameter in (6-16) takes into account the flux normalization uncertainties and in order to deal with it we will introduce a pull term $\chi_{\text {pull }}^{2}$ which we define as

$$
\chi_{\text {pull }}^{2}=\frac{(\beta-1)^{2}}{\sigma_{\beta}^{2}}
$$

where we consider a flux uncertainty of the order of $\sigma_{\beta}=2 \%$. The process to 


\section{Appearance $v$ mode}

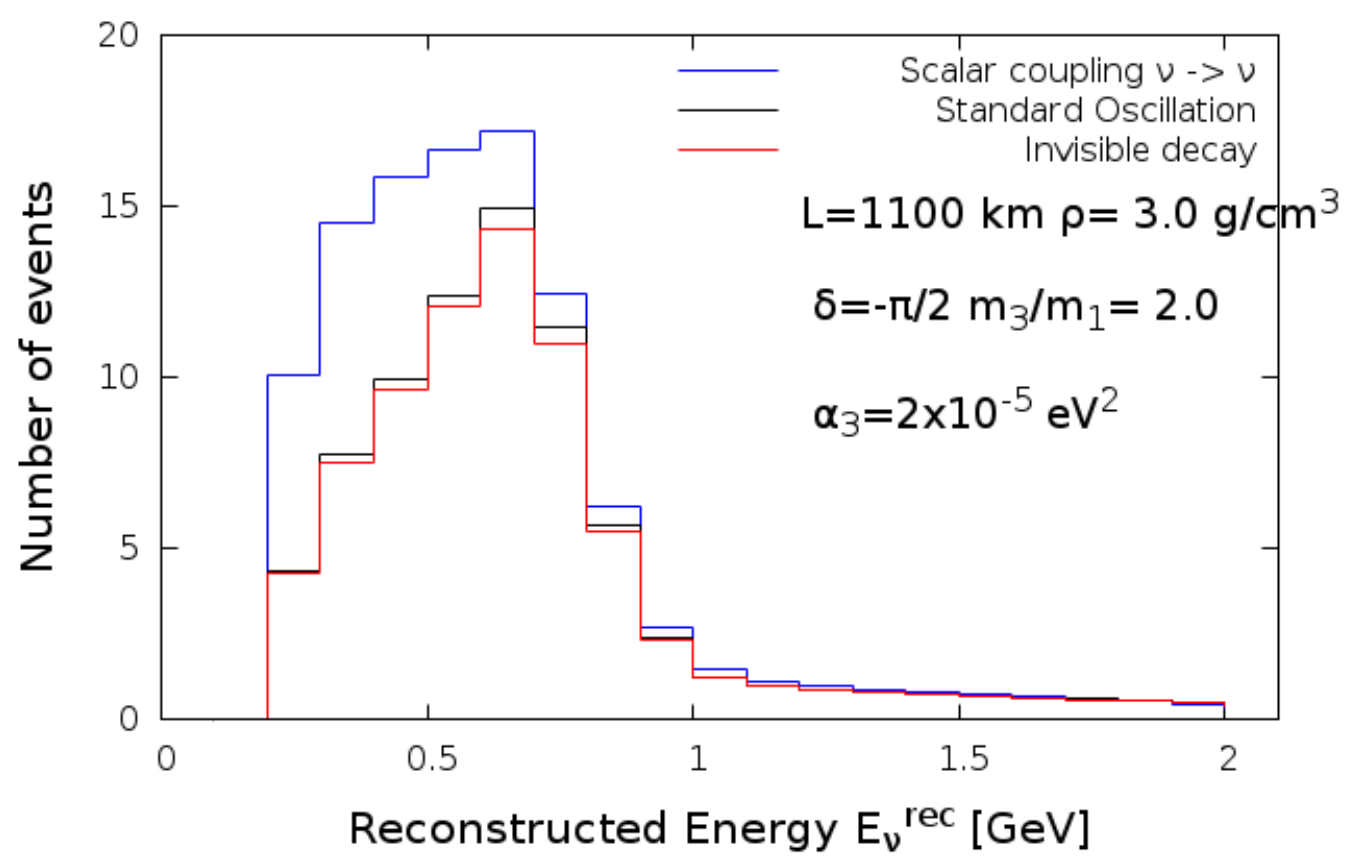

Figure 6.11: Expected events number distribution for the appearance channel for T2HK for $\nu_{\mu} \rightarrow \nu_{e}$ for the case of standard oscillation against invisible decay and scalar coupling helicity conserving visible decay effect. For both cases we used $\alpha_{3}=5 \times 10^{-5} \mathrm{eV}^{2}$ and for the visible case the mass ratio $m_{3} / m_{1}=2.0$.

realize the minimization of the $\chi^{2}$ function is done as following: our first step is to marginalize the $\chi^{2}$ with respect to $\beta$

$$
\chi_{\text {eff }}^{2}\left(m_{\text {light }}, \Delta m_{31}^{2}, \sin _{23}^{2}, \delta_{C P}, \alpha_{3}, \beta\right)=\min _{(\beta)} \chi^{2}\left(m_{\text {light }}, \Delta m_{31}^{2}, \sin _{23}^{2}, \delta_{C P}, \alpha_{3}\right) .
$$

Once we get the $\chi_{\text {eff }}^{2}$ we pass to minimize with respect to all other parameters except for $\alpha_{3}$.

\section{4}

\section{Analysis Results}

\subsection{1}

\section{Visible Case}

Here we show our results for the visible decay case for all the couplings (scalar and pseudo-scalar) and for conserving and non conserving helicity for the T2HKK setup. In Fig. 6.12 and Fig. 6.13 are shown the results for the disappearance channel $\nu_{\mu} \rightarrow \nu_{\mu}$ and $\bar{\nu}_{\mu} \rightarrow \bar{\nu}_{\mu}$. As we expected this channel is not so sensible to the $\alpha_{3}$ variation so that our best results $\alpha_{3}<8.2 \times 10^{-6} \mathrm{eV}^{2} 90 \%$ C.L. is worse then the limits we are comparing to. 


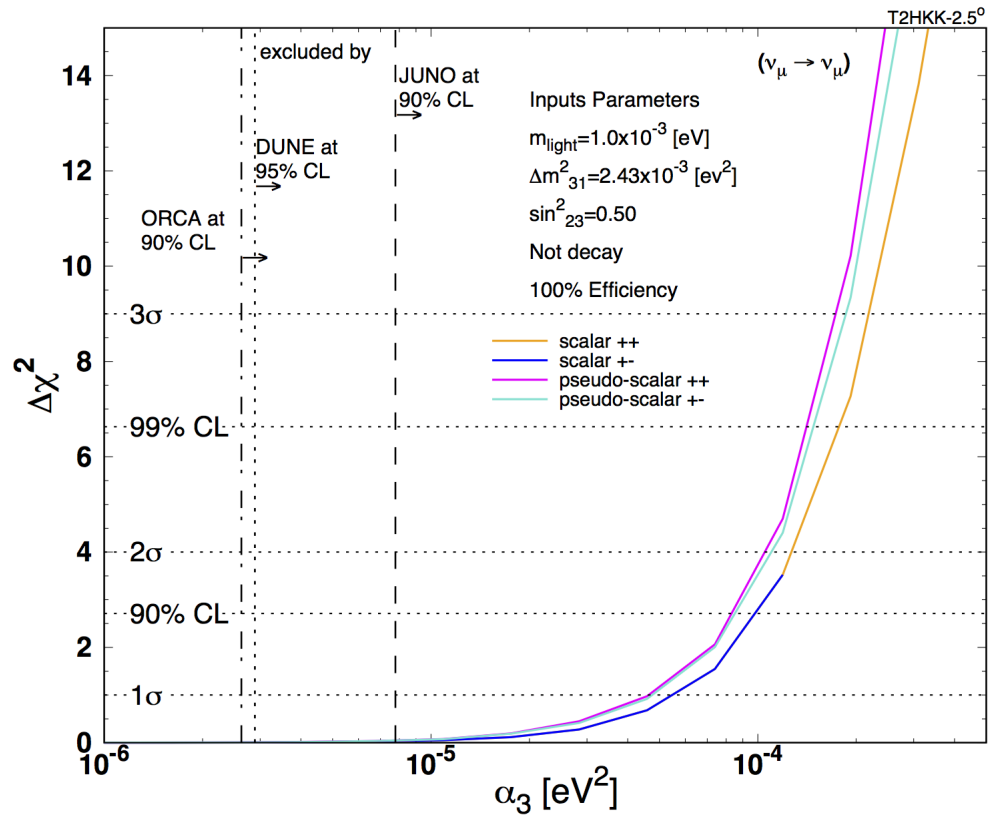

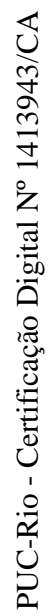

Figure 6.12: Expected sensitivity obtained for the $\nu_{\mu} \rightarrow \nu_{\mu}$ channel. We can exclude with $90 \%$ C.L. at best $\alpha_{3}<8.4 \times 10^{-5} \mathrm{eV}^{2}$ for the case of a pseudoscalar coupling in the helicity conserving case.

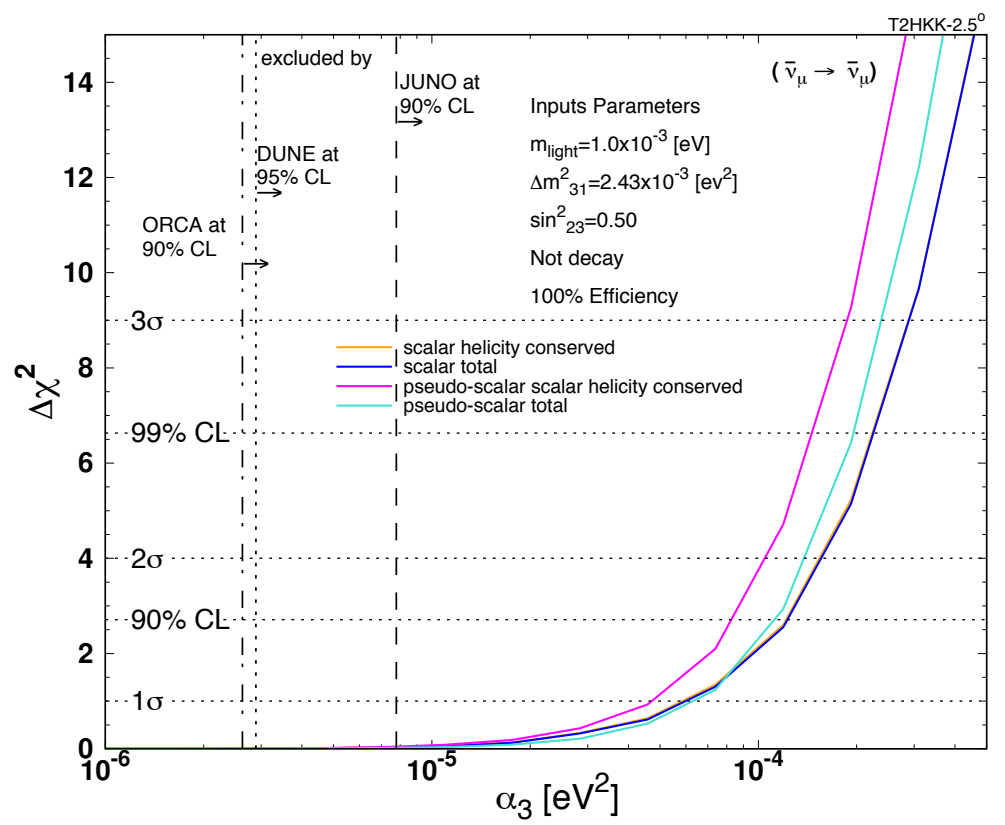

Figure 6.13: Expected sensitivity obtained for the $\bar{\nu}_{\mu} \rightarrow \bar{\nu}_{\mu}$ channel. We can exclude with $90 \%$ C.L. at best $\alpha_{3}<8.2 \times 10^{-5} \mathrm{eV}^{2}$ for the case of a pseudoscalar coupling in the helicity conserving case. 


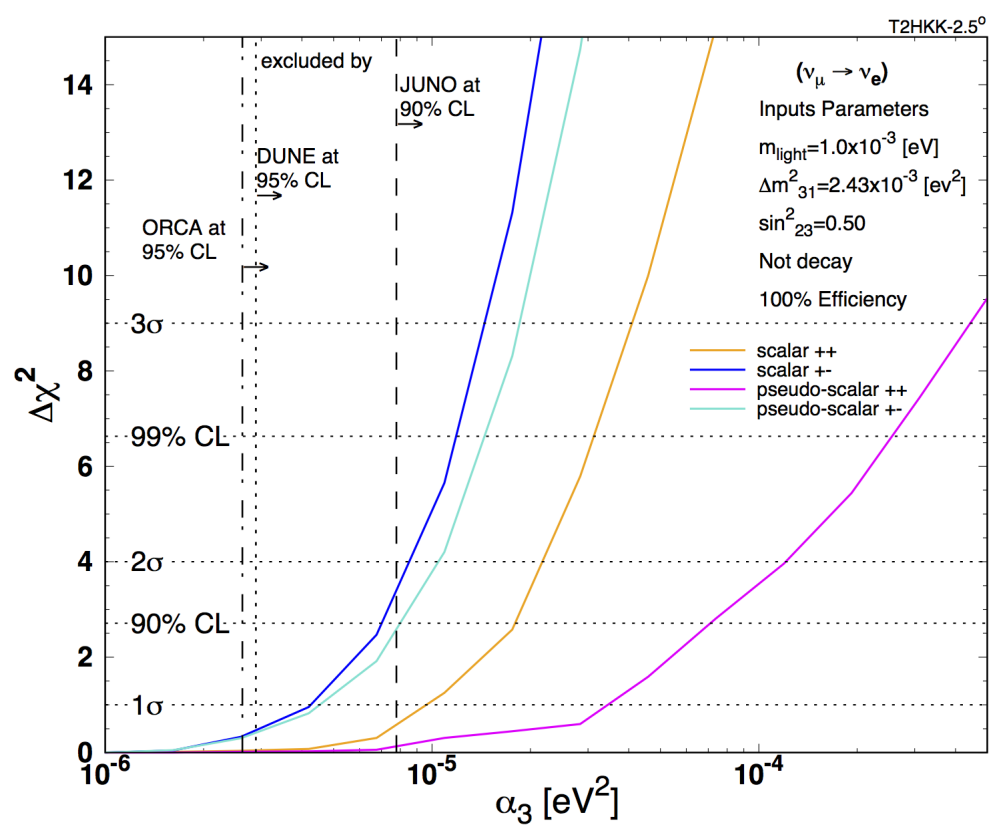

Figure 6.14: Expected sensitivity obtained for the $\nu_{\mu} \rightarrow \nu_{e}$ channel. We can exclude with $90 \%$ C.L. at best $\alpha_{3}<7.0 \times 10^{-6} \mathrm{eV}^{2}$ for the case of a scalar coupling in the helicity non conserving case.

On the other hand for the appearance channel $\nu_{\mu} \rightarrow \nu_{e}$ and $\bar{\nu}_{\mu} \rightarrow \bar{\nu}_{e}$ we have a better results. Our best result $\alpha_{3}<7.0 \times 10^{-6} \mathrm{eV}^{2} 90 \%$ C.L. for $\nu_{\mu} \rightarrow \nu_{e}$ channel is better than the one from [38]. 


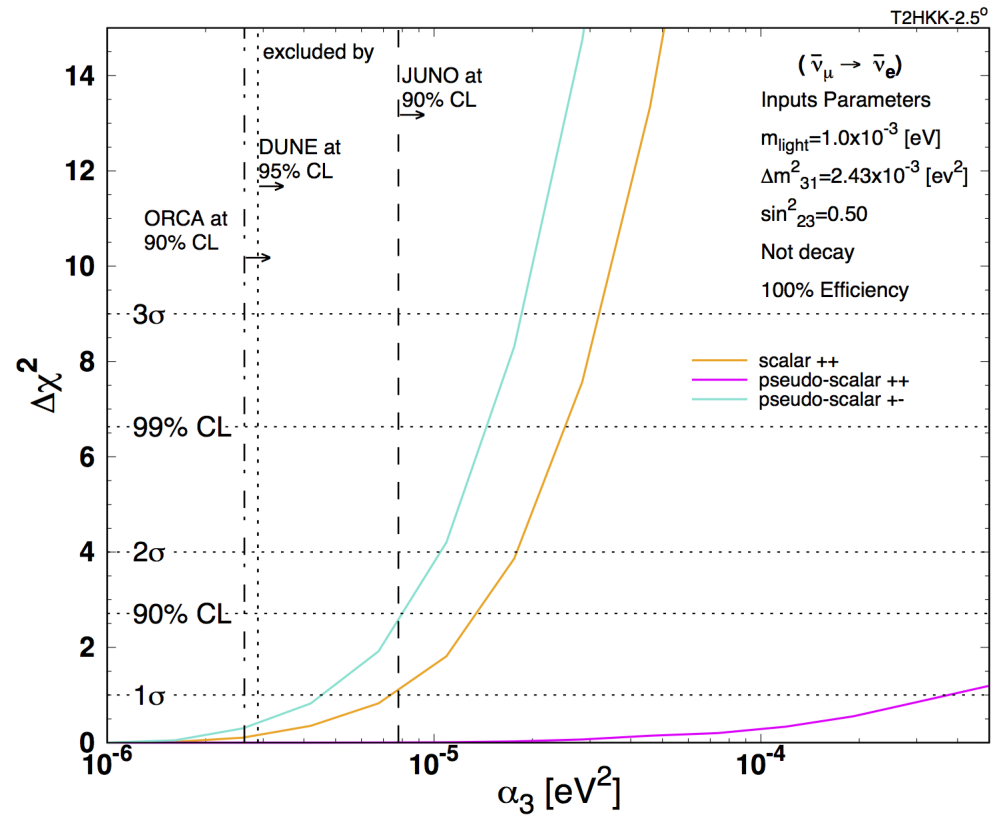

Figure 6.15: Expected sensitivity obtained for the $\bar{\nu}_{\mu} \rightarrow \bar{\nu}_{e}$ channel. We can exclude with $90 \%$ C.L. at best $\alpha_{3}<8.0 \times 10^{-6} \mathrm{eV}^{2}$ for the case of a pseudoscalar coupling in the helicity non conserving case. 


\section{Conclusions}

In this thesis we discussed how new generation neutrino experiments can measure the leptonic $\mathrm{CP}$ violation phase and test a possible neutrino decay scenario.

In the first part of the thesis we have found out that the new experimental setup, called LiquidO, can determine the $\mathrm{CP} \delta$ phase with a sensitivity comparable with experiments like DUNE and HK. We determined that for a $\mathrm{B}$ factor of 30 , that corresponds to

$$
B[30] \Rightarrow 300 \mathrm{kt} \times 10 \text { years } \times 5 \mathrm{MW} \text { beam } \times 10 \% \text { proton fraction, }
$$

and for $\eta=0.3$, i.e. $15 \%$ of loading fraction, we can cover $37 \%$ of all the possible $\delta$ true values with a confidence level $\geq 5 \sigma$. We also studied the correlation between $\delta$ and $\sin ^{2} \theta_{23}$ in LiquidO setup.

In the second part of the thesis we performed a preliminary analysis for the T2HKK experiment in the case of $\nu_{3} \rightarrow \nu_{1}+\phi$ visible decay scenario. Our calculation pointed out that in neutrino mode, for the appearance channel we can exclude at $90 \%$ C.L. $\alpha_{3}<7.0 \times 10^{-6} \mathrm{eV}^{2}$, or in terms of neutrino lifetime $\tau_{3} / m_{3}=1.05 \times 10^{-13} \mathrm{~s} / \mathrm{eV}$. This result is quite positive considering that we are considering unfavorable $m_{3} / m_{1}$ ratio and we can still add T2HK analysis. In conclusion we expect to improve even further our limit. 
A

\section{Higgs Mechanism and Flavour Mixing}

In the SM the mechanism that allows fermions, as well as the $W^{ \pm}$ and $Z$ bosons, to acquire masses is the Higgs mechanism. This mechanism was inspired by Nambu and Goldstone in 1960s, that first investigated the consequences of a Spontaneous Symmetry Breaking (SSB) in condensed matter physics. To better describe the concept of the SSB in quantum field theory it is useful to introduce a classical physics analogy: consider a thin cylindrical bar placed vertically on a table with an external force $F$ directed along its axis as shown in Fig.A.1.

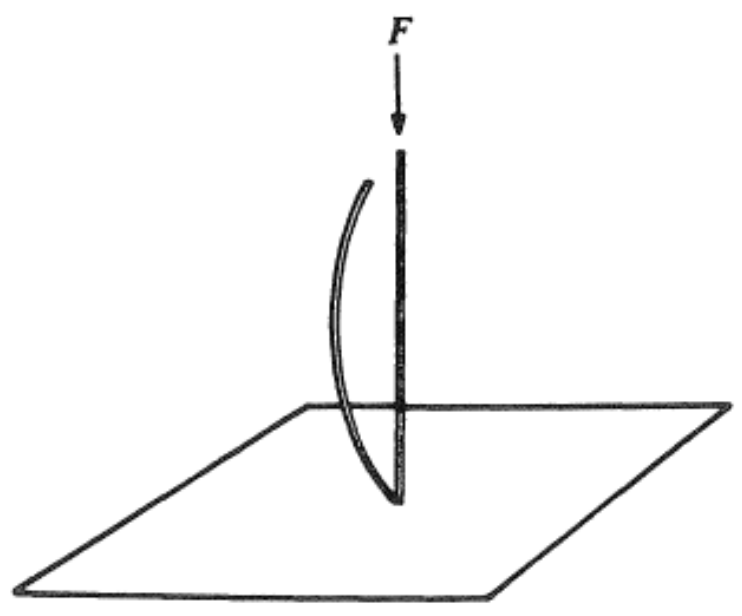

Figure A.1: A thin bar bent under an external force that breaks the initial $U(1)$ symmetry of the system.

If the module of $F$ is small nothing happens, but if $F>F_{\text {crit }}$ the bar will bend in a direction choose at random. The initial $U(1)$ symmetry of the bar has been broken and the new "ground state" is one of the infinite possible ones all related to one another by a rotation (the action of an element of $U(1)$ ). As one can see this simple example contains all the crucial point of the SSB

1) The initial state, i.e. ground state, is symmetric and the configuration becomes unstable because of the variation of a parameter.

2) After the external parameter reach a critical value the ground state looses its initial symmetry. 
3) The new ground state is degenerate, i.e. can be related by other ground state by a transformation.

Now in the Higgs mechanism the ground state is the Vacuum Expectation Value (VEV) of a scalar neutral field $\left\langle 0\left|\phi^{0}\right| 0\right\rangle=v$, the external parameter is introduced by a coupling in the potential $V(\phi)$ and the initial symmetry of the Higgs field (in the electroweak case the $S U(2)_{L} \times U(1)_{Y}$ ) is broken to a $U(1)_{Q}$ one. To have a more rigorous definition of all these quantities let us introduce the Higgs doublet

$$
\boldsymbol{\Phi}=\left(\begin{array}{l}
\phi^{+} \\
\phi^{0}
\end{array}\right)
$$

where $\phi^{+}$is a positive charged complex scalar field and $\phi^{0}$ is neutral complex scalar field. The gauge quantum numbers of the Higgs fields are listed in Fig.A.2.

\begin{tabular}{llcccc}
\hline & $I$ & $I_{3}$ & $Y$ & $Q$ \\
\cline { 3 - 5 } Higgs doublet & $\Phi(x) \equiv\left(\begin{array}{c}\phi^{+}(x) \\
\phi^{0}(x)\end{array}\right)$ & $1 / 2$ & $1 / 2$ & & 1 \\
& & $-1 / 2$ & & 0 \\
\hline
\end{tabular}

Figure A.2: The Higgs double eigenvalues respect to all the generators that fix the action of an element of $S U(2)_{L} \times U(1)_{Y}$.

The corresponding Lagrangian associated to the Higgs fields is

$$
\mathscr{L}_{\text {Higgs }}=\left(D_{\mu} \boldsymbol{\Phi}\right)^{\dagger}\left(D^{\mu} \boldsymbol{\Phi}\right)-V(\boldsymbol{\Phi})
$$

where $D_{\mu}$ is defined in (2-20) and

$$
V(\boldsymbol{\Phi})=\mu^{2} \boldsymbol{\Phi}^{\dagger} \boldsymbol{\Phi}+\lambda\left(\boldsymbol{\Phi}^{\dagger} \boldsymbol{\Phi}\right)^{2} .
$$

If we allow the coupling $\mu^{2}$ to assume negative values (the external parameter) the potential will be modified like in Fig.A.3 making the previous minimum unstable and provide the SSB

$$
S U(2)_{L} \times U(1)_{Y} \rightarrow U(1)_{Q}
$$

Defining

the potential is minimum for

$$
v \equiv \sqrt{-\frac{\mu^{2}}{\lambda}}
$$

$$
\boldsymbol{\Phi}^{\dagger} \boldsymbol{\Phi}=\frac{v^{2}}{2}
$$




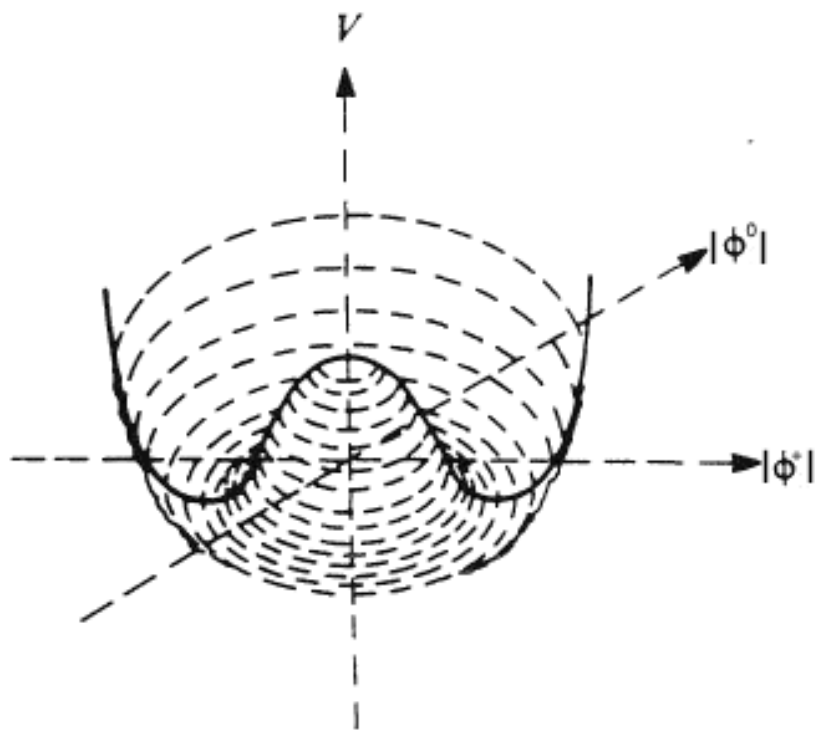

Figure A.3: The potential $V(\boldsymbol{\Phi})$ has a minimum in $\boldsymbol{\Phi}^{\dagger} \boldsymbol{\Phi}=v^{2} / 2$ and a local minimum in $\boldsymbol{\Phi}^{\dagger} \boldsymbol{\Phi}=0$.

This expression in quantum field theory defines the VEV. Fermion and vector boson fields, which carry nonzero spin, must have a zero value in the vacuum, in order to preserve the manifest invariance of Nature under spatial rotation. Also, charged scalar fields must have zero value in the vacuum, which is electrically neutral. On the other hand, neutral scalar fields, which do not have electric charge, can have a nonzero value in vacuum. In order to respect all these physical principles the VEV of the Higgs field can be written as

$$
\langle\boldsymbol{\Phi}\rangle=\frac{1}{\sqrt{2}}\left(\begin{array}{l}
0 \\
v
\end{array}\right) .
$$

This VEV now naturally breaks the $S U(2)_{L} \times U(1)_{Y}$ symmetry and is just invariant under a $U(1)$ action that implies the existence of a massless boson, i.e. the electromagnetic photon. To explore the physics consequences of the SSB we can rewrite the Higgs field as

$$
\boldsymbol{\Phi}=\frac{1}{\sqrt{2}}\left(\begin{array}{c}
0 \\
v+H(x)
\end{array}\right),
$$

where the real 4-field $H(x)$ describes the physical Higgs boson, obtained by excitations of the neutral Higgs field above the vacuum.

Let us consider now the Lagrangian mass terms of quarks, for example the term relatives to the $d, s, b$ quarks like we defined in (2-9)

$$
\overline{Q_{\alpha L}} q_{\beta R}^{\prime D},
$$


where $\alpha=1,2,3$ runs over the generation, $\beta=d, s, b$ runs over the flavours and the $D(U)$ indicates the quarks that have $I_{3}=-(+) 1 / 2$. This term has hypercharge $Y=-1$ and can be coupled to the Higgs doublet A-8

$$
-\sum_{\alpha=1,2,3} \sum_{\beta=d, s, b} Y_{\alpha \beta}^{\prime D} \overline{Q_{\alpha L}} \boldsymbol{\Phi} q_{\beta R}^{\prime D}
$$

where $Y_{\alpha \beta}^{\prime}$ is a complex $3 \times 3$ complex matrix of Yukawa couplings. This type of mass term is analogous to the leptonic ones and has the same physics characteristics. Putting the physics Higgs we defined before into A-10

$$
-\left(\frac{x+H}{\sqrt{2}}\right) \sum_{\alpha, \beta=d, s, b} Y_{\alpha \beta}^{\prime D} \overline{q_{\alpha L}} q_{\beta R}^{\prime D},
$$

so that the part of this summation proportional to $v$ is the one responsible for the mass. In general the Yukawa coupling matrix is not diagonal and in order to define properly the mass term we need to rearrange the primed fields in order to diagonalize this complex matrix

$$
\begin{gathered}
q_{\beta R}^{D}=V_{R}^{D^{\dagger}} q_{\beta R}^{\prime D} \quad q_{\beta L}^{\prime D}=V_{L}^{D^{\dagger}} q_{\beta L}^{\prime}, \\
V_{L}^{D^{\dagger}} Y_{\alpha \beta}^{\prime D} V_{R}^{D}=y_{\alpha}^{D} \delta_{\alpha \beta} .
\end{gathered}
$$

Now the unprimed fields have definite mass and so are the physical fields

$$
-\sum_{\alpha=d, s, b} \frac{v y_{\alpha}^{D}}{\sqrt{2}} \overline{q_{\alpha L}^{D}} q_{\alpha R}^{D},
$$

where one can recognize

$$
\frac{v y_{\alpha}^{D}}{\sqrt{2}}
$$

Obviously this redefinition of the fields must be applied to all Lagrangian's terms and when it comes to the interaction terms it manifests in the form of flavour mixing. In fact, in the charged interaction terms there is no Yukawa coupling matrix and so what happen is

$$
j_{W}^{\mu}=2 \overline{q_{L}^{\prime}} \gamma^{\mu} q_{\beta L}^{\prime D}=2 \gamma^{\mu} \overline{q_{L}^{\bar{U}}} V_{L}^{U \dagger} V_{L}^{D} \gamma^{\mu} q_{\beta L}^{D},
$$

wehre $V_{C K M}=V_{L}^{U^{\dagger}} V_{L}^{D}$ is the Cabibbo-Kobayashi-Maskawa (CKM) matrix, which embodies the physical effects of quark mixing. This matrix is unitary because product of two unitary matricies and any unitary matrix can be considered a representation of the group $S U(n)$ with $n=2$ in the case of three generation. This matrix can be parametrized in many ways but the standard one [1] (that is used as well in the leptonic analogous) is defined by 3 angles and 1 complex phase 


$$
V_{C K M}=\left(\begin{array}{ccc}
c_{12} c_{13} & s_{12} c_{13} & s_{13} e^{\mathrm{i} \delta} \\
-s_{12} c_{23}-c_{12} s_{23} s_{13} e^{\mathrm{i} \delta} & c_{12} c_{23}-s_{12} s_{23} s_{13} e^{\mathrm{i} \delta} & s_{23} c_{13} \\
s_{12} s_{23}-c_{12} c_{23} s_{13} e^{\mathrm{i} \delta} & -c_{12} s_{23}-s_{12} c_{23} s_{13} e^{\mathrm{i} \delta} & c_{23} c_{13}
\end{array}\right)
$$

with $c_{i j}=\cos \theta_{i j}, s_{i j}=\sin \theta_{i j}$ and $\delta$ beeing the Dirac phase that as I showed in Chapter 2 plays a fundamental rule in the CP-violation. 


\section{B}

\section{Visible Decay Calculation}

Here we summarize the relevant formulas used in Chapter 6 . The visible contribution for the $\nu_{\alpha} \rightarrow \nu_{\beta}$ channel in the presence of the matter effect is given by [43]

$$
\begin{aligned}
& \frac{\mathrm{d} P_{v i s}^{\nu_{\alpha}^{A} \rightarrow \nu_{\beta}^{B}}\left(E_{\nu_{\alpha}}, E_{\nu_{\beta}}\right)}{\mathrm{d} E_{\nu_{\beta}}}= \int_{0}^{L} \mathrm{~d} l \mid \sum_{I=1}^{3} \sum_{J=1}^{3} \sum_{i=1}^{3} \sum_{j=1}^{i-1} \tilde{C}_{I i}^{A}\left(\tilde{U}^{(A)}\right)_{I \alpha}^{-1}\left(\hat{C}^{(B)}\right)_{J j}^{-1} \hat{U}_{\beta J}^{(B)} \\
& \times \exp \left[-\mathrm{i} \frac{\tilde{m}_{I}^{2} l}{2 E_{\nu_{\alpha}}}\right] \exp \left[-\frac{\tilde{\alpha}_{I} l}{2 E_{\nu_{\alpha}}}\right] \exp \left[-\mathrm{i} \frac{\hat{m}_{J}^{2}(L-l)}{2 E_{\nu_{\beta}}}\right] \exp \left[-\frac{\hat{\alpha}_{J}(L-l)}{2 E_{\nu_{\beta}}}\right] \\
& \times\left.\sqrt{\frac{\mathrm{d}}{\mathrm{d} E_{\nu_{\beta}}} \Gamma_{\nu_{i}^{A} \rightarrow \nu_{j}^{B}}\left(E_{\nu_{\alpha}}\right)}\right|^{2}
\end{aligned}
$$

where

$$
\tilde{C}_{I j}^{(A)}=\sum_{\rho=e, \mu, \tau} \tilde{U}_{\rho I}^{(A)} U_{\rho j}^{(A) *} \quad \hat{C}_{I j}^{(A)}=\sum_{\rho=e, \mu, \tau} \hat{U}_{\rho I}^{(A)} U_{\rho j}^{(A) *}
$$

Following the notations of [43], matrices $\tilde{U}$ and $\tilde{C}(\hat{U}$ and $\hat{C})$ correspond to the ones related to parent (daughter) neutrino $\nu^{A}\left(\nu^{B}\right)$ where $A$ and $B$ $( \pm)$ indicate the neutrino helicity. Since we are considering the case where $\nu_{3} \rightarrow \nu_{1}+\phi$, Eq. (B-1) can be simplified as follows,

$$
\frac{\mathrm{d} P_{v i s}^{\nu_{\alpha}^{A} \rightarrow \nu_{\beta}^{B}}\left(E_{\nu_{\alpha}}, E_{\nu_{\beta}}\right)}{\mathrm{d} E_{\nu_{\beta}}}=Q_{\alpha \beta}^{A B}\left(E_{\nu_{\alpha}}, E_{\nu_{\beta}}\right) \frac{\mathrm{d}}{\mathrm{d} E_{\nu_{\beta}^{B}}} \Gamma_{\nu_{3}^{A} \rightarrow \nu_{1}^{B}}\left(E_{\nu_{\alpha}}, E_{\nu_{\beta}}\right),
$$

where

$$
\begin{aligned}
& Q_{\alpha \beta}^{A B}\left(E_{\nu_{\alpha}}, E_{\nu_{\beta}}\right) \equiv \sum_{I=1}^{3} \sum_{J=1}^{3} \sum_{M=1}^{3} \sum_{N=1}^{3}\left(\tilde{U}^{(A)}\right)_{I \alpha}^{-1}\left(\tilde{U}^{(A)}\right)_{M \alpha}^{-1 *} \hat{U}_{\beta J}^{(B)} \hat{U}_{\beta N}^{(B) *} \tilde{C}_{I 3}^{(A)} \tilde{C}_{M 3}^{(A) *}\left(\hat{C}^{(B)}\right)_{1 J}^{-1}\left(\hat{C}^{(B)}\right)_{1 N}^{-1 *} \\
& 2 E_{\nu_{\beta}} \times \frac{\left[\left(E_{\nu_{\beta}} / E_{\nu_{\alpha}}\right) \tilde{\alpha}_{<I M>}-\hat{\alpha}_{<J N>}\right]-\mathrm{i}\left[\left(E_{\nu_{\beta}} / E_{\nu_{\alpha}}\right) \Delta \tilde{m}_{I M}^{2}-\Delta \hat{m}_{J N}^{2}\right]}{\left[\left(E_{\nu_{\beta}} / E_{\nu_{\alpha}}\right) \tilde{\alpha}_{<I M>}-\hat{\alpha}_{<J N>}\right]^{2}+\left[\left(E_{\nu_{\beta}} / E_{\nu_{\alpha}}\right) \Delta \tilde{m}_{I M}^{2}-\Delta \hat{m}_{J N}^{2}\right]^{2}} \\
& \times\left\{\exp \left[-\mathrm{i} \frac{\Delta \hat{m}_{J N}^{2} L}{2 E_{\nu_{\beta}}}\right] \exp \left[-\frac{\hat{\alpha}_{<J N>} L}{2 E_{\nu_{\beta}}}\right]-\exp \left[-\mathrm{i} \frac{\Delta \tilde{m}_{I M}^{2} L}{2 E_{\nu_{\alpha}}}\right] \exp \left[-\frac{\tilde{\alpha}_{<I M>} L}{2 E_{\nu_{\alpha}}}\right]\right\},
\end{aligned}
$$


and where $\tilde{\alpha}_{<I M>} \equiv \tilde{\alpha}_{I}+\tilde{\alpha}_{M}, \Delta \hat{m}_{J N}^{2}=\tilde{m}_{J}^{2}-\tilde{m}_{N}^{2}$ as well as for the $\hat{\cdot}$ quantities. In vacuum $Q_{\alpha \beta}^{A B}\left(E_{\nu_{\alpha}}, E_{\nu_{\beta}}\right)$ are given as

$$
Q_{\alpha \beta}^{A B}\left(E_{\nu_{\alpha}}, E_{\nu_{\beta}}\right)=\left|U_{\alpha 3}^{(A)}\right|^{2}\left|U_{\beta 1}^{(B)}\right|^{2}\left(\frac{E_{\nu_{\alpha}}}{\alpha_{3}}\right)\left[1-\exp \left(-\frac{\alpha_{3}}{E_{\nu_{\alpha}} L}\right)\right]
$$

Note that in vacuum, $Q_{\alpha \beta}^{A B}\left(E_{\nu_{\alpha}}, E_{\nu_{\beta}}\right)$ does not depends on the energy of daughter neutrino. For small value of $\alpha_{3} L / E_{\nu_{\alpha}} \ll 1, Q_{\alpha \beta}^{A B}\left(E_{\nu_{\alpha}}, E_{\nu_{\beta}}\right)$ does not depend also on parent neutrino energy and is given by

$$
Q_{\alpha \beta}^{A B} \simeq 1.5(5.6) \times 10^{12}\left|U_{\alpha 3}^{(A)}\right|^{2}\left|U_{\beta 1}^{(B)}\right|^{2}\left[\frac{L}{295(1100) \mathrm{km}}\right] \mathrm{eV}^{-1} .
$$

In Fig. B.1 we show how the functions $Q_{\mu \mu}\left(E_{\nu_{\mu}}^{0}, E_{\nu_{\mu}}\right)$ and $Q_{\mu e}\left(E_{\nu_{\mu}}^{0}, E_{e}\right)$ behave for the case where $\alpha_{3}=5 \times 10^{-5} \mathrm{eV}^{2}$ and $\delta=-\pi / 2$ for four different helicity decay modes. We observe that the matter effect for $\mathrm{L}=295 \mathrm{~km}$ is very small (four curves with matter effect almost coincide with that for the vacuum case) whereas that for $L=1100 \mathrm{~km}$ has some impact though not very large.

The function $\frac{\mathrm{d}}{\mathrm{d} E_{\nu_{\beta}^{B}}} \Gamma_{\nu_{3}^{A} \rightarrow \nu_{1}^{B}}\left(E_{\nu_{\alpha}}, E_{\nu_{\beta}}\right)$ is given by

$$
\frac{\mathrm{d}}{\mathrm{d} E_{\nu_{\beta}^{B}}} \Gamma_{\nu_{3}^{A} \rightarrow \nu_{1}^{B}}\left(E_{\nu_{\alpha}}, E_{\nu_{\beta}}\right)=\frac{\alpha_{3}}{E_{\nu_{\alpha}}^{2}} \frac{x_{31}^{2}}{\left(x_{31}^{2}-1\right)} \Theta\left(x_{31}^{2} E_{\nu_{\beta}}-E_{\nu_{\alpha}}\right) F_{g}^{A B}\left(E_{\nu_{\alpha}}, E_{\nu_{\beta}}\right),
$$

where $x_{i f}=m_{i} / m_{f}$ and the dimensionless functions $F_{g}^{A B}\left(E_{\nu_{\alpha}}, E_{\nu_{\beta}}\right)$ qre given [43],

$$
\begin{aligned}
& F_{g_{s}}^{ \pm \pm}\left(E_{\nu_{\alpha}}, E_{\nu_{\beta}}\right)=\frac{1}{E_{\nu_{\alpha}} E_{\nu_{\beta}}} \frac{\left(E_{\nu_{\alpha}}+x_{i f} E_{\nu_{\beta}}\right)^{2}}{\left(x_{i f}+1\right)^{2}}, \\
& F_{g_{s}}^{ \pm \mp}\left(E_{\nu_{\alpha}}, E_{\nu_{\beta}}\right)=\frac{\left(E_{\nu_{\alpha}}-E_{\nu_{\beta}}\right)}{E_{\nu_{\alpha}} E_{\nu_{\beta}}} \frac{\left(x_{i f}^{2} E_{\nu_{\beta}}+E_{\nu_{\alpha}}\right)^{2}}{\left(x_{i f}+1\right)^{2}}, \\
& F_{g_{p}}^{ \pm \pm}\left(E_{\nu_{\alpha}}, E_{\nu_{\beta}}\right)=\frac{1}{E_{\nu_{\alpha}} E_{\nu_{\beta}}} \frac{\left(E_{\nu_{\alpha}}-x_{i f} E_{\nu_{\beta}}\right)^{2}}{\left(x_{i f}-1\right)^{2}}, \\
& F_{g_{p}}^{ \pm \mp}\left(E_{\nu_{\alpha}}, E_{\nu_{\beta}}\right)=\frac{\left(E_{\nu_{\alpha}}-E_{\nu_{\beta}}\right)}{E_{\nu_{\alpha}} E_{\nu_{\beta}}} \frac{\left(x_{i f}^{2} E_{\nu_{\beta}}+E_{\nu_{\alpha}}\right)^{2}}{\left(x_{i f}-1\right)^{2}},
\end{aligned}
$$

where $A$ and $B$ indicate the helicity of parent and daughter neutrino, respectively, denoted as $+(-)$ for positive (negative) helicity, and $g_{s}\left(g_{p}\right)$ implies the case of scalar (pseudo-scalar) coupling. Note that $F_{g_{s}}^{ \pm \pm}\left(E_{\nu_{\alpha}}, E_{\nu_{\beta}}\right)+$ $F_{g_{s}}^{ \pm \mp}\left(E_{\nu_{\alpha}}, E_{\nu_{\beta}}\right)=1$. The typical dependence of the functions $\Gamma_{\nu_{3}^{A} \rightarrow \nu_{1}^{B}}\left(E_{\nu_{\alpha}}, E_{\nu_{\beta}}\right)$ on $E_{\nu_{\beta}}$ are shown in Fig. B.2 for the case $E_{\nu_{\alpha}}=3 \mathrm{GeV}$ 

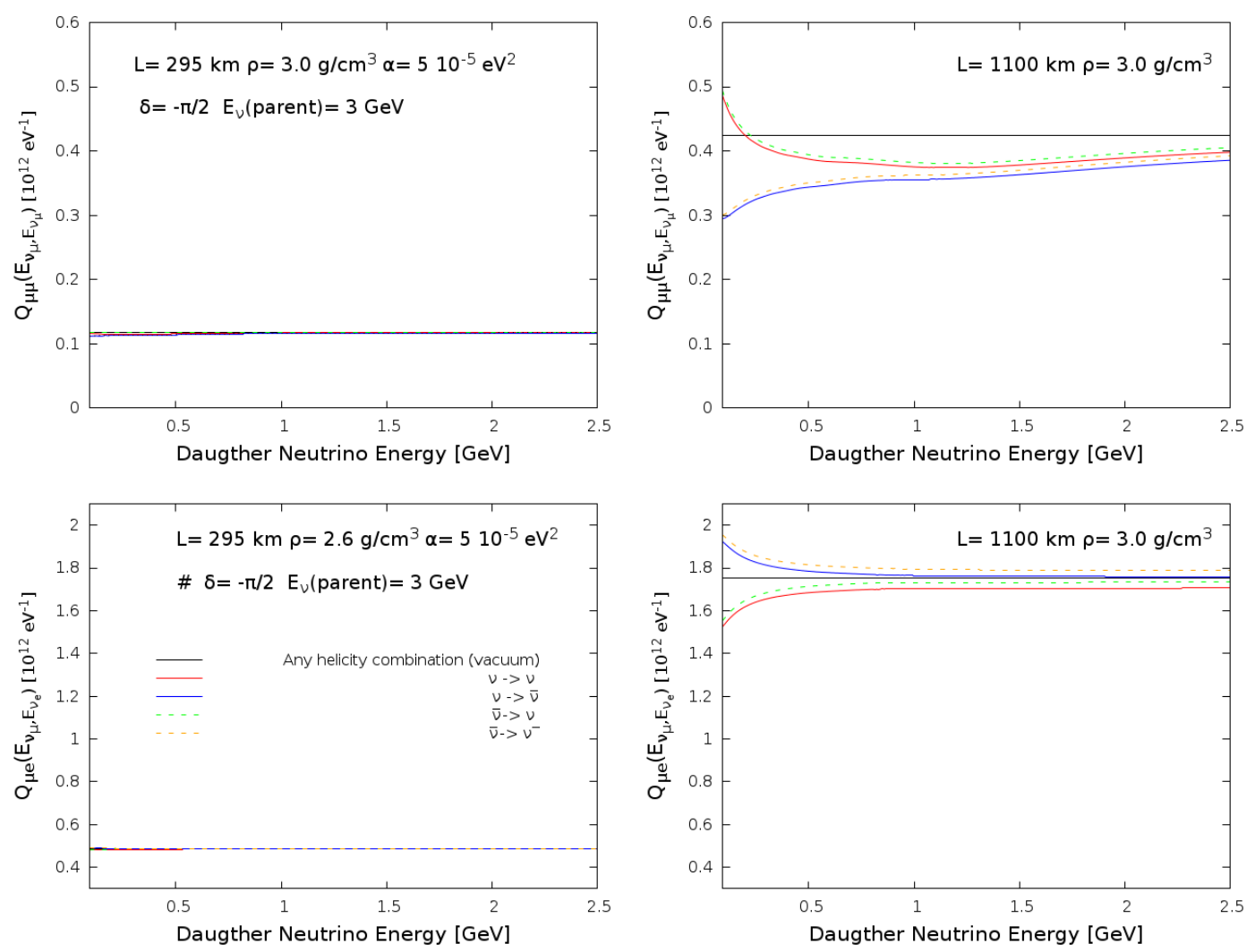

Figure B.1: The function defined in Eq.(B-4), $Q_{\mu \mu}\left(E_{\nu_{\mu}}^{0}, E_{\nu_{\mu}}\right)$ and $Q_{\mu e}\left(E_{\nu_{\mu}}^{0}, E_{e}\right)$ for four different helicity decay modes are shown, respectively, in the upper and lower panels as a function of the neutrino energy for $L=295 \mathrm{~km}$ (left panels) and $L=1100 \mathrm{~km}$ (right panels) in the presence (absence) of matter effect with $2.6 \mathrm{~g} / \mathrm{cm}^{3}\left(3.0 \mathrm{~g} / \mathrm{cm}^{3}\right)$ in the presence of the decay effect with $\alpha_{3}=5 \times 10^{-5} \mathrm{eV}^{2}$. It is shown for the cases where the energies of parent neutrino are $3.0 \mathrm{GeV}$ with and the CP phase is taken $\delta=-\pi / 2$. 

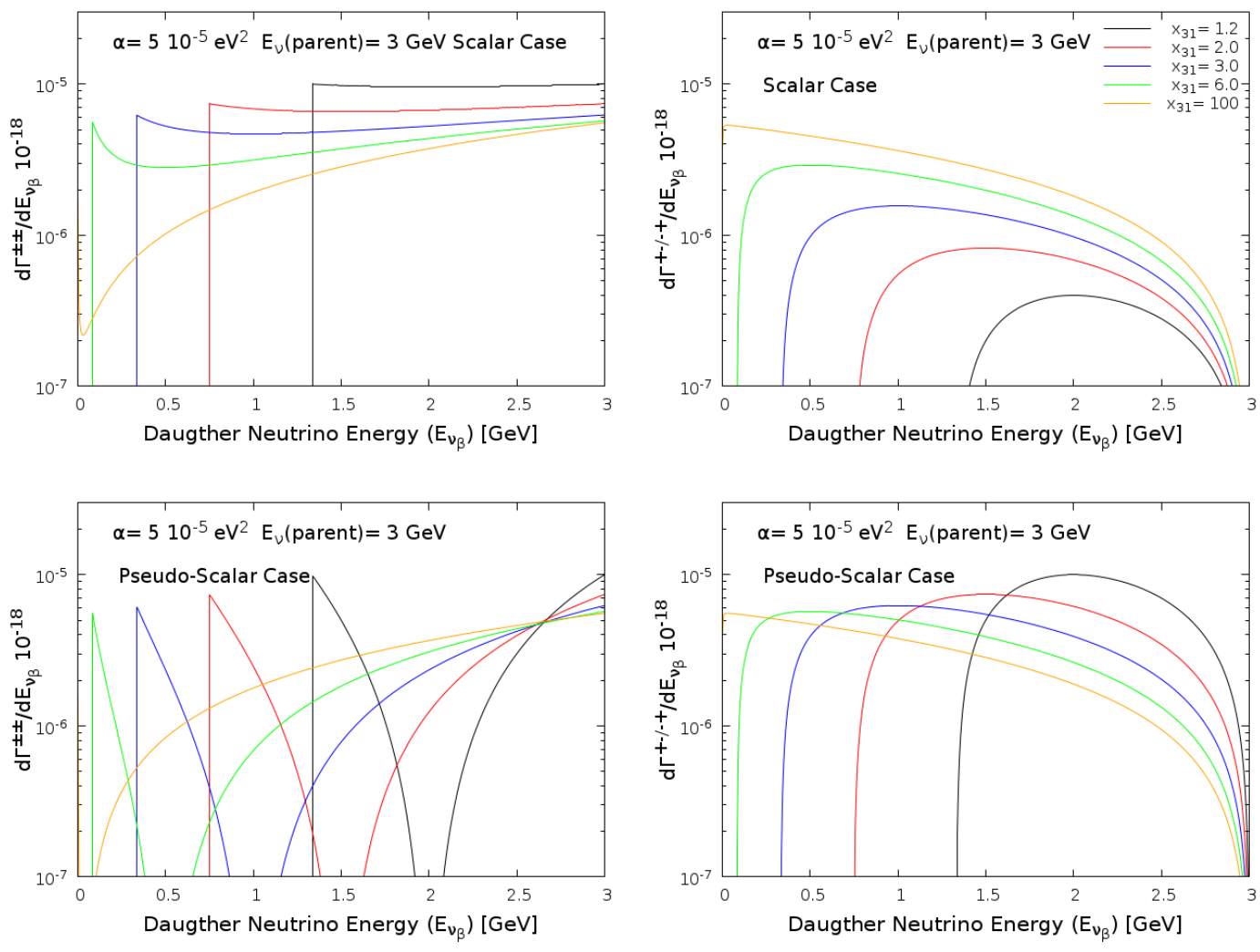

Figure B.2: The $\Gamma_{\nu_{3}^{A} \rightarrow \nu_{1}^{B}}\left(E_{\nu_{\alpha}}, E_{\nu_{\beta}}\right)$ as a function of the daugther neutrino energy $E_{\nu_{\beta}}$. We show in the upper (lower) panels the case of scalar (pseudoscalar) coupling for helicity conserving case (left column) and helicity nonconserving case (right column) for $\alpha_{3}=5 \times 10^{-5} \mathrm{eV}^{2}$ and a fixed parent neutrino energy $E_{\nu_{\alpha}}=3 \mathrm{GeV}$. 


\section{C \\ Calculations of Number of Events for T2HK and T2HKK}

The general expression to calculate the expected number of events in the case of neutrino decay is calculated as follows,

$$
\frac{\mathrm{d} N\left(E_{\mathrm{rec}}\right)}{\mathrm{d} E_{\mathrm{rec}}}=n T \sigma_{0} \int \mathrm{d} E_{\nu_{\beta}} \sum_{\text {helicity }} \frac{\mathrm{d} \phi_{\nu_{\beta}^{B}}^{\text {osc }+ \text { decay }}\left(E_{\nu_{\beta}^{B}}\right)}{\mathrm{d} E_{\nu_{\beta}^{B}}} \epsilon\left(E_{\nu_{\beta}}\right) \sigma\left(E_{\nu_{\beta}}\right) R\left(E_{\nu_{\beta}}, E_{\mathrm{rec}}\right),
$$

where $n$ is the number of target in the fiducial volume, $T$ is the exposure time, $\sigma_{0}=10^{-32} \mathrm{~cm}^{2} / \mathrm{GeV}, \epsilon$ is the detection efficiency, $\sigma$ is the cross section shape function and $\mathrm{d} \phi_{\nu_{\beta}^{B}}^{\text {osc }+ \text { decay }}\left(E_{\nu_{\beta}^{B}}\right) / \mathrm{d} E_{\nu_{\beta}^{B}}$ is the differential neutrino flux spectra expected at the detector site (which includes oscillation and decay effects) computed as in Eq. (6-12). $R\left(E_{\nu_{\beta}}, E_{\text {rec }}\right)$ is is the Gaussian resolution function, given by

$$
R\left(E_{\nu_{\beta}}, E_{\mathrm{rec}}\right)=\frac{1}{2 \pi \sigma\left(E_{\nu_{\beta}}\right)} \exp \left[-\left(\frac{E_{\nu_{\beta}}-\left(E_{\mathrm{rec}}-E_{\mathrm{shift}}\left(E_{\nu_{\beta}}\right)\right)}{\sigma\left(E_{\nu_{\beta}}\right)}\right)^{2}\right],
$$

where $E_{\text {shift }}\left(E_{\nu_{\beta}}\right)=c+d E_{\nu_{\beta}}$ is the energy shift in the energy reconstruction and $\sigma\left(E_{\nu_{\beta}}\right)=a+b \sqrt{E_{\nu_{\beta}}}$ is the square root of the quadratic variance (also called width). This resolution function is fitted on Monte Carlo simulations of HK events and so a,b,c and d fit parameters are determined for QE and nQE interactions. In order to do so I took this information directly from [45] in the table showed in Fig.C.1.

\begin{tabular}{|c|c|c|c|c|}
\hline & \multicolumn{2}{|c|}{$0.55 \mathrm{GeV}$} & \multicolumn{2}{c|}{$0.75 \mathrm{GeV}$} \\
\hline & width $(\mathrm{MeV})$ & shift $(\mathrm{MeV})$ & width $(\mathrm{MeV})$ & shift $(\mathrm{MeV})$ \\
\hline$\nu \mathrm{QE}$ & 85 & -10 & 98 & -15 \\
\hline$\nu \mathrm{nQE}$ & 70 & -325 & 110 & -390 \\
\hline $\bar{\nu} \mathrm{QE}$ & 57 & -20 & 60 & -20 \\
\hline $\bar{\nu} \mathrm{nQE}$ & 100 & -270 & 120 & -310 \\
\hline
\end{tabular}

Figure C.1: Fitted width and shift for different values of $E_{\nu}$ and for different interaction type.

In our simulation we also consider $\epsilon\left(E_{\nu_{\beta}}\right)$ as a constant with respect to the energy with its value depending of the interaction type and the channel (appearance or disappearance). This values are reported for the case of the 
appearance channel (the type of events for this channel are also called $R \mu$ ) in Fig. C.2 that is a table took from [16]. The same has been done for the appearance channel (the type of events for this channel are also called $R e$ ) where we took the efficiencies from C.3.

\begin{tabular}{cccccccccc}
\hline \hline & & $\nu_{\mu} \mathrm{CCQE}$ & $\nu_{\mu} \mathrm{CC}$ non-QE & $\bar{\nu}_{\mu} \mathrm{CCQE}$ & $\bar{\nu}_{\mu} \mathrm{CC}$ non-QE & $\nu_{e}+\bar{\nu}_{e} \mathrm{CC}$ & $\mathrm{NC}$ & $\nu_{\mu} \rightarrow \nu_{e}$ & total \\
\hline \multirow{2}{*}{$\nu$ mode } & Events & 8460 & 4173 & 487 & 271 & 9 & 672 & 40 & 14110 \\
& Eff. (\%) & 91.0 & 20.7 & 95.6 & 53.5 & 0.5 & 8.8 & 1.1 & - \\
\hline \multirow{2}{*}{$\bar{\nu}$ mode } & Events & 3778 & 3295 & 8539 & 2745 & 10 & 844 & 5 & 19214 \\
& Eff. (\%) & 88.0 & 20.1 & 95.4 & 54.8 & 0.4 & 8.8 & 0.7 & - \\
\hline \hline
\end{tabular}

Figure C.2: The expected number of $\nu_{\mu} / \bar{\nu}_{\mu}$ events and efficiencies for each flavour and interaction type

\begin{tabular}{cc|cc|ccccc|c|c}
\hline \hline & & \multicolumn{2}{|c|}{ signal } & \multicolumn{7}{c|}{ BG } \\
& & $\nu_{\mu} \rightarrow \nu_{e}$ & $\bar{\nu}_{\mu} \rightarrow \bar{\nu}_{e}$ & $\nu_{\mu} \mathrm{CC}$ & $\bar{\nu}_{\mu} \mathrm{CC}$ & $\nu_{e} \mathrm{CC}$ & $\bar{\nu}_{e} \mathrm{CC}$ & $\mathrm{NC}$ & BG Total & Total \\
\hline \multirow{2}{*}{$\nu$ mode } & Events & 2300 & 21 & 10 & 0 & 347 & 15 & 188 & 560 & 2880 \\
& Eff.(\%) & 63.6 & 47.3 & 0.1 & 0.0 & 24.5 & 12.6 & 1.4 & 1.6 & - \\
\hline \multirow{2}{*}{$\bar{\nu}$ mode } & Events & 289 & 1656 & 3 & 3 & 142 & 302 & 274 & 724 & 2669 \\
& Eff. (\%) & 45.0 & 70.8 & 0.03 & 0.02 & 13.5 & 30.8 & 1.6 & 1.6 & - \\
\hline \hline
\end{tabular}

Figure C.3: The expected number of $\nu_{e} / \bar{\nu}_{e}$ events and efficiencies for each flavour and interaction type

The explicit version of $(\mathrm{C}-1)$ for the disappearance channel is

$$
\begin{aligned}
\frac{\mathrm{d} N^{R \mu}\left(E_{\mathrm{rec}}\right)^{ \pm \pm}}{\mathrm{d} E_{\mathrm{rec}}} & =n T \sigma_{0} \int_{0.05 \mathrm{GeV}}^{\infty} \mathrm{d} E_{\nu_{\beta}} \frac{\mathrm{d} \phi_{\nu_{\mu} \rightarrow \nu_{\mu}}^{\mathrm{osc}+\text { decay }}\left(E_{\nu_{\beta}^{B}}\right)}{\mathrm{d} E_{\nu_{\beta}^{B}}}\left\{\sigma_{Q E}\left(E_{\nu_{\beta}}\right) \epsilon_{Q E} R_{Q E}\left(E_{\nu}, E_{\mathrm{rec}}\right)\right. \\
& \left.+\sigma_{n Q E}\left(E_{\nu_{\beta}}\right) \epsilon_{n Q E} R_{n Q E}\left(E_{\nu_{\beta}}, E_{\mathrm{rec}}\right)\right\} \\
& \left.+\sigma_{N C}\left(E_{\nu_{\beta}}\right) \epsilon_{N C}\right\} \\
& +\frac{\mathrm{d} \phi_{\bar{\nu}_{\mu} \rightarrow \bar{\nu}_{\mu}}^{\text {osc }+ \text { decay }}\left(E_{\nu_{\beta}^{B}}\right)}{\mathrm{d} E_{\nu_{\beta}^{B}}}\left\{\sigma_{Q E}\left(E_{\nu_{\beta}}\right) \epsilon_{Q E} R_{Q E}\left(E_{\nu_{\beta}}, E_{\mathrm{rec}}\right)\right. \\
& \left.+\sigma_{n Q E}\left(E_{\nu_{\beta}}\right) \epsilon_{n Q E} R_{n Q E}\left(E_{\nu_{\beta}}, E_{\mathrm{rec}}\right)\right\} \\
& \left.+\sigma_{N C}\left(E_{\nu_{\beta}}\right) \epsilon_{N C}\right\},
\end{aligned}
$$

where this is the helicity conservative case that stands for the standard oscillation case or the invisible decay case. For the non conservative helicity 
case we added the term

$$
\begin{aligned}
\frac{\mathrm{d} N^{R \mu}\left(E_{\mathrm{rec}}\right)^{ \pm \mp(\mp \pm)}}{\mathrm{d} E_{\mathrm{rec}}} & =n T \sigma_{0} \int_{0.05 \mathrm{GeV}}^{\infty} \mathrm{d} E_{\nu_{\beta}} \frac{\mathrm{d} \phi_{\bar{\nu}_{\mu} \rightarrow \nu_{\mu}}^{\text {osc }+ \text { decay }}\left(E_{\nu_{\beta}^{B}}\right)}{\mathrm{d} E_{\nu_{\beta}^{B}}}\left\{\sigma_{Q E}\left(E_{\nu_{\beta}}\right) \epsilon_{Q E} R_{Q E}\left(E_{\nu}, E_{\mathrm{rec}}\right)\right. \\
& \left.+\sigma_{n Q E}\left(E_{\nu_{\beta}}\right) \epsilon_{n Q E} R_{n Q E}\left(E_{\nu_{\beta}}, E_{\mathrm{rec}}\right)\right\} \\
& \left.+\sigma_{N C}\left(E_{\nu_{\beta}}\right) \epsilon_{N C}\right\} \\
& +\frac{\mathrm{d} \phi_{\nu_{\mu} \rightarrow \bar{\nu}_{\mu}}^{\text {osc }+ \text { decay }}\left(E_{\nu_{\beta}^{B}}\right)}{\mathrm{d} E_{\nu_{\beta}^{B}}}\left\{\sigma_{Q E}\left(E_{\nu_{\beta}}\right) \epsilon_{Q E} R_{Q E}\left(E_{\nu_{\beta}}, E_{\mathrm{rec}}\right)\right. \\
& \left.+\sigma_{n Q E}\left(E_{\nu_{\beta}}\right) \epsilon_{n Q E} R_{n Q E}\left(E_{\nu_{\beta}}, E_{\mathrm{rec}}\right)\right\} \\
& \left.+\sigma_{N C}\left(E_{\nu_{\beta}}\right) \epsilon_{N C}\right\} .
\end{aligned}
$$

It is important to note that in our analysis for the disappearance channel we did not consider the background coming from the $\nu_{e}$ produced at the source because their contribution is $\sim 0.07 \%$ (see C.2). For the appearance case we can not apply the same assumption because the $\nu_{e}$ contamination is much bigger, i.e. $\sim 9 \%$. In this case we have for the helicity conserving case

$$
\begin{aligned}
\frac{\mathrm{d} N^{R e}\left(E_{\mathrm{rec}}\right)^{ \pm \pm}}{\mathrm{d} E_{\mathrm{rec}}} & =n T \sigma_{0} \int_{0.05 \mathrm{GeV}}^{\infty} \mathrm{d} E_{\nu_{\beta}} \frac{\mathrm{d} \phi_{\nu_{\mu} \rightarrow \nu_{e}}^{\text {osc }+ \text { decay }}\left(E_{\nu_{\beta}^{B}}\right)}{\mathrm{d} E_{\nu_{\beta}^{B}}}\left\{\sigma_{Q E}\left(E_{\nu_{\beta}}\right) \epsilon_{Q E} R_{Q E}\left(E_{\nu}, E_{\mathrm{rec}}\right)\right. \\
& \left.+\sigma_{N C}\left(E_{\nu_{\beta}}\right) \epsilon_{N C}\right\} \\
& +\frac{\mathrm{d} \phi_{\bar{\nu}_{\mu} \rightarrow \bar{\nu}_{e}}^{\text {osc }+ \text { decay }}\left(E_{\nu_{\beta}^{B}}\right)}{\mathrm{d} E_{\nu_{\beta}^{B}}}\left\{\sigma_{Q E}\left(E_{\nu_{\beta}}\right) \epsilon_{Q E} R_{Q E}\left(E_{\nu_{\beta}}, E_{\mathrm{rec}}\right)+\sigma_{N C}\left(E_{\nu_{\beta}}\right) \epsilon_{N C}\right\} \\
& +\frac{\mathrm{d} \phi_{\bar{\nu}_{e} \rightarrow \bar{\nu}_{e}}^{\text {osc }+ \text { decay }}\left(E_{\nu_{\beta}^{B}}\right)}{\mathrm{d} E_{\nu_{\beta}^{B}}}\left\{\sigma_{Q E}\left(E_{\nu_{\beta}}\right) \epsilon_{Q E} R_{Q E}\left(E_{\nu_{\beta}}, E_{\mathrm{rec}}\right)+\sigma_{N C}\left(E_{\nu_{\beta}}\right) \epsilon_{N C}\right\} \\
& +\frac{\mathrm{d} \phi_{\nu_{e} \rightarrow \nu_{e}}^{\text {osc }+ \text { decay }}\left(E_{\nu_{\beta}^{B}}\right)}{\mathrm{d} E_{\nu_{\beta}^{B}}}\left\{\sigma_{Q E}\left(E_{\nu_{\beta}}\right) \epsilon_{Q E} R_{Q E}\left(E_{\nu_{\beta}}, E_{\mathrm{rec}}\right)+\sigma_{N C}\left(E_{\nu_{\beta}}\right) \epsilon_{N C}\right\},
\end{aligned}
$$

and for the non conserving helicity case

$$
\begin{aligned}
\frac{\mathrm{d} N^{R e}\left(E_{\mathrm{rec}}\right)^{ \pm \mp(\mp \pm)}}{\mathrm{d} E_{\mathrm{rec}}} & =n T \sigma_{0} \int_{0.05 \mathrm{GeV}}^{\infty} \mathrm{d} E_{\nu_{\beta}} \frac{\mathrm{d} \phi_{\bar{\nu}_{\mu} \rightarrow \nu_{e}}^{\text {osc }+ \text { decay }}\left(E_{\nu_{\beta}^{B}}\right)}{\mathrm{d} E_{\nu_{\beta}^{B}}}\left\{\sigma_{Q E}\left(E_{\nu_{\beta}}\right) \epsilon_{Q E} R_{Q E}\left(E_{\nu}, E_{\mathrm{rec}}\right)\right. \\
& \left.+\sigma_{N C}\left(E_{\nu_{\beta}}\right) \epsilon_{N C}\right\} \\
& +\frac{\mathrm{d} \phi_{\nu_{\mu} \rightarrow \bar{\nu}_{e}}^{\text {osc }}\left(E_{\nu_{\beta}^{B}}\right)}{\mathrm{d} E_{\nu_{\beta}^{B}}}\left\{\sigma_{Q E}\left(E_{\nu_{\beta}}\right) \epsilon_{Q E} R_{Q E}\left(E_{\nu_{\beta}}, E_{\mathrm{rec}}\right)+\sigma_{N C}\left(E_{\nu_{\beta}}\right) \epsilon_{N C}\right\} \\
& +\frac{\mathrm{d} \phi_{\nu_{e} \rightarrow \bar{\nu}_{e}}^{\text {osc }+ \text { decay }}\left(E_{\nu_{\beta}^{B}}\right)}{\mathrm{d} E_{\nu_{\beta}^{B}}}\left\{\sigma_{Q E}\left(E_{\nu_{\beta}}\right) \epsilon_{Q E} R_{Q E}\left(E_{\nu_{\beta}}, E_{\mathrm{rec}}\right)+\sigma_{N C}\left(E_{\nu_{\beta}}\right) \epsilon_{N C}\right\} \\
& +\frac{\mathrm{d} \phi_{\bar{\nu}_{e} \rightarrow \nu_{e}}^{\text {osc }+ \text { decay }}\left(E_{\nu_{\beta}^{B}}\right)}{\mathrm{d} E_{\nu_{\beta}^{B}}}\left\{\sigma_{Q E}\left(E_{\nu_{\beta}}\right) \epsilon_{Q E} R_{Q E}\left(E_{\nu_{\beta}}, E_{\mathrm{rec}}\right)+\sigma_{N C}\left(E_{\nu_{\beta}}\right) \epsilon_{N C}\right\},
\end{aligned}
$$




\section{Bibliography}

[1] PATRIGNANI, C.; OTHERS. Review of Particle Physics. Chin. Phys., C40(10):100001, 2016.

[2] GIUNTI, C.; KIM, C. W.. Fundamentals of neutrino physics and astrophysics. Oxford University Press, 2015.

[3] STRUMIA, A.; VISSANI, F.. Precise quasielastic neutrino/nucleon cross-section. Physics Letters B, 564(1):42 - 54, 2003.

[4] DAVIS, JR., R.; HARMER, D. S. ; HOFFMAN, K. C.. Search for neutrinos from the sun. Phys. Rev. Lett., 20:1205-1209, 1968.

[5] BAHCALL, J. N.; PINSONNEAULT, M. H. ; BASU, S.. Solar models: Current epoch and time dependences, neutrinos, and helioseismological properties. The Astrophysical Journal, 555(2):990, 2001.

[6] AHMAD, Q. R.; ET AL., A.. Direct evidence for neutrino flavor transformation from neutral-current interactions in the sudbury neutrino observatory. Phys. Rev. Lett., 89:011301, Jun 2002.

[7] EGUCHI, K.; ET AL., E.. First results from kamland: Evidence for reactor antineutrino disappearance. Phys. Rev. Lett., 90:021802, Jan 2003.

[8] TANABASHI, M.; OTHERS. Review of particle physics. Phys. Rev. D, 98:030001, Aug 2018.

[9] KAYSER, B.. Neutrino physics. eConf, C040802, hep-ph/0506165:L004, 2004.

[10] WOLFENSTEIN, L.. Neutrino oscillations in matter. Phys. Rev. D, 17:2369-2374, May 1978.

[11] ZAGlaueR, H. W.; SCHWARZER, K. H.. The Mixing Angles in Matter for Three Generations of Neutrinos and the Msw Mechanism. Z. Phys., C40:273, 1988. 
[12] MIKHEEV, S. P.; SMIRNOV, A. YU.. Resonant amplification of neutrino oscillations in matter and solar neutrino spectroscopy. Nuovo Cim., C9:17-26, 1986.

[13] JARLSKOG, C.. Commutator of the quark mass matrices in the standard electroweak model and a measure of maximal $\mathrm{CP}$ nonconservation. Phys. Rev. Lett., 55:1039-1042, Sep 1985.

[14] PONTECORVO, B.. Neutrino Experiments and the Problem of Conservation of Leptonic Charge. Sov. Phys. JETP, 26:984-988, 1968. [Zh. Eksp. Teor. Fiz.53,1717(1967)].

[15] MALTONI, M.; SMIRNOV, A. YU.. Solar neutrinos and neutrino physics. Eur. Phys. J., A52(4):87, 2016.

[16] ABE, K.; OTHERS. Hyper-Kamiokande Design Report. arXiv:1805.04163 [physics.ins-det], 2018.

[17] FEngPeng, A.; OTHERS. Neutrino Physics with JUNO. J. Phys., G43(3):030401, 2016.

[18] ANDRINGA, S.; OTHERS. Current Status and Future Prospects of the SNO+ Experiment. Adv. High Energy Phys., 2016:6194250, 2016.

[19] WENDELL, R.; OKUMURA, K.. Recent progress and future prospects with atmospheric neutrinos. New J. Phys., 17(2):025006, 2015.

[20] KAJITA, T.. Nobel lecture: Discovery of atmospheric neutrino oscillations. Rev. Mod. Phys., 88:030501, Jul 2016.

[21] WASCKO, M.. T2k status, results, and plans. Talk Presented at XXVIII International Conference on Neutrino Physics and Astrophysics - (Neutrino 2018), Heidelberg, June 4-9, 2018, avilable at https://doi.org/10.5281/zenodo.1286752, June 2018.

[22] SANCHEZ, M.. Nova results and prospects. Talk Presented at XXVIII International Conference on Neutrino Physics and Astrophysics - (Neutrino 2018), Heidelberg, June 4-9, 2018, avilable at https://doi.org/10.5281/zenodo.1286758, June 2018.

[23] ACCIARRI, R.; OTHERS. Long-Baseline Neutrino Facility (LBNF) and Deep Underground Neutrino Experiment (DUNE). arXiv:1601.05471 [physics.ins-det], 2016. 
[24] ABE, K.; OTHERS. Physics potentials with the second HyperKamiokande detector in Korea. PTEP, 2018(6):063C01, 2018.

[25] QIAN, X.; PENG, J.-C.. Physics with Reactor Neutrinos. Rept. Prog. Phys., 82:036201, 2019.

[26] NUNOKAWA, H.; PARKE, S. ; FUNCHAL, R. Z.. Another possible way to determine the neutrino mass hierarchy. Phys. Rev. D, 72:013009, Jul 2005.

[27] ESTEBAN, I.; GONZALEZ-GARCIA, M. C.; MALTONI, M.; MARTINEZSOLER, I. ; SCHWETZ, T.. Updated fit to three neutrino mixing: exploring the accelerator-reactor complementarity. JHEP, 01:087, 2017.

[28] NUNOKAWA, H.. Resolving the octant theta(23) degeneracy by neutrino oscillation experiments. Nucl. Phys. Proc. Suppl., 168:212214, 2007. [,212(2006)].

[29] HAGEDORN, C.; MOHAPATRA, R. N.; MOLINARO, E.; NISHI, C. C. ; PETCOV, S. T.. CP Violation in the Lepton Sector and Implications for Leptogenesis. Int. J. Mod. Phys., A33(05n06):1842006, 2018.

[30] NUNOKAWA, H.; PARKE, S. J. ; VALLE, J. W. F.. CP Violation and Neutrino Oscillations. Prog. Part. Nucl. Phys., 60:338-402, 2008.

[31] AN, F. P.; ET AL., B.. Observation of electron-antineutrino disappearance at daya bay. Phys. Rev. Lett., 108:171803, Apr 2012.

[32] MINAKATA, H.; NUNOKAWA, H.. How to measure CP violation in neutrino oscillation experiments? Phys. Lett., B413:369-377, 1997.

[33] CONRAD, J. M.; SHAEVITZ, M. H.. Multiple cyclotron method to search for $c p$ violation in the neutrino sector. Phys. Rev. Lett., 104:141802, Apr 2010.

[34] SCHOLBERG, K.. Supernova neutrino detection. Annual Review of Nuclear and Particle Science, 62(1):81-103, 2012.

[35] FORMAGgIO, J. A.; ZELLER, G. P.. From ev to eev: Neutrino cross sections across energy scales. Rev. Mod. Phys., 84:1307-1341, Sep 2012. 
[36] HARADA, M.; OTHERS. Proposal: A Search for Sterile Neutrino at J-PARC Materials and Life Science Experimental Facility. arXiv:1310.1437 [physics.ins-det], 2013.

[37] BerRymAN, J. M.; DE GOUVEA, A. ; HERnANDEZ, D.. Solar Neutrinos and the Decaying Neutrino Hypothesis. Phys. Rev., D92(7):073003, 2015.

[38] ABRAHÃO, T.; MINAKATA, H.; NUNOKAWA, H. ; QUIROGA, A. A.. Constraint on Neutrino Decay with Medium-Baseline Reactor Neutrino Oscillation Experiments. JHEP, 11:001, 2015.

[39] COLOMA, P.; PERES, O. L. G.. Visible neutrino decay at DUNE. arXiv:1705.03599 [hep-ph], 2017.

[40] DE SALAS, P. F.; PASTOR, S.; TERNES, C. A.; THAKORE, T. ; TÓRTOLA, M.. Constraining the invisible neutrino decay with KM3NeTORCA. Phys. Lett., B789:472-479, 2019.

[41] SCHECHTER, J.; VALLE, J. W. F.. Neutrino decay and spontaneous violation of lepton number. Phys. Rev. D, 25:774-783, Feb 1982.

[42] BERRYMAN, J. M.; DE GOUVÊA, A. ; HERNÁNDEZ, D.. Solar neutrinos and the decaying neutrino hypothesis. Phys. Rev. D, 92:073003, Oct 2015.

[43] GAGO, A, M.; OTHERS. Visible neutrino decay in the light of appearance and disappearance long-baseline experiments. Journal of High Energy Physics, 2017(11):22, Nov 2017.

[44] FUKUDA, Y.; OTHERS. Evidence for oscillation of atmospheric neutrinos. Phys. Rev. Lett., 81:1562-1567, Aug 1998.

[45] MACHADO, P. A. N.; MINAKATA, H.; NUNOKAWA, H. ; ZUKANOVICH FUNCHAL, R.. What can we learn about the lepton CP phase in the next 10 years? JHEP, 05:109, 2014. 